\title{
Application of the emotional intelligence construct to college student binge drinking
}

Jeffrey P. Dulko

West Virginia University

Follow this and additional works at: https://researchrepository.wvu.edu/etd

\section{Recommended Citation}

Dulko, Jeffrey P., "Application of the emotional intelligence construct to college student binge drinking" (2007). Graduate Theses, Dissertations, and Problem Reports. 2592.

https://researchrepository.wvu.edu/etd/2592

This Dissertation is protected by copyright and/or related rights. It has been brought to you by the The Research Repository @ WVU with permission from the rights-holder(s). You are free to use this Dissertation in any way that is permitted by the copyright and related rights legislation that applies to your use. For other uses you must obtain permission from the rights-holder(s) directly, unless additional rights are indicated by a Creative Commons license in the record and/ or on the work itself. This Dissertation has been accepted for inclusion in WVU Graduate Theses, Dissertations, and Problem Reports collection by an authorized administrator of The Research Repository @ WVU.

For more information, please contact researchrepository@mail.wvu.edu. 


\title{
Application of the Emotional Intelligence Construct To College Student
} Binge Drinking

\author{
Jeffrey P. Dulko
}

Dissertation submitted to the College of Human Resources and Education

at West Virginia University in partial fulfillment of the requirements

for the degree of

\author{
Doctor of Philosophy \\ in \\ Counseling Psychology \\ Margaret K. Glenn, Ed.D., Chair \\ Lynda Birckhead Danley, Ph.D. \\ Richard T. Walls, Ph.D. \\ James Bartee, Ph.D. \\ Ed Jacobs, Ph.D. \\ Department of Counseling, Rehabilitation Counseling, \\ and Counseling Psychology
}

\section{Morgantown, WV}

2007

Keywords: binge drinking, alcohol-related consequences, emotional intelligence, alcohol prevention programs

Copyright 2007 Jeffrey P. Dulko 


\author{
ABSTRACT \\ Application of the Emotional Intelligence Construct to \\ College Student Binge Drinking \\ Jeffrey P. Dulko
}

Although extensive research has been conducted in the area of college binge drinking, relatively little has been done to study differences among alcoholrelated consequences. Some initial studies have shown that binge drinking and its consequences are associated with several emotional and interpersonal factors, but there has been no comprehensive examination of these relationships. The theory of emotional intelligence (EI) has been developed recently to study emotional and interpersonal factors as an integrated construct. Research of the relationship between alcohol and EI could provide a valuable base for which to design alcohol prevention programs that target improving emotional and interpersonal deficits as a means to reduce consequences. The present study used three surveys to assess binge drinking and to examine how EI and its factors can predict binge drinking consequences. The Drinker Inventory of Consequences (DrInC), the BarOn Emotional Intelligence Quotient (EQ-i), and a Research Questionnaire were administered to 309 undergraduate college students. The results revealed that there is no significant difference between binge drinkers and non-binge drinkers on a measure of general EI. However, the number of binge drinking consequences is inversely correlated with general EI. All five types of binge drinking consequences (physical, intrapersonal, interpersonal, impulse control, and social responsibility) were predicted by EI factors (intrapersonal, stress management, and interpersonal). As intrapersonal EI rose, physical consequences from binge drinking decreased. Higher stress management EI scores were indicative of lower intrapersonal binge drinking consequences, and higher interpersonal EI scores predicted lower social responsibility consequences. A combination of higher levels of both stress management and interpersonal EI coincided with separate reports of lower interpersonal and impulse control consequences. Gender differences observed among the EI factors for binge drinkers suggest that males and females may have unique EI deficits that could influence specific types of consequences. The findings have implications for further research of alcohol prevention curricula on college campuses that address the improvement of specific EI deficits as a means to lowering binge drinking consequences. 


\section{Dedication}

For my grandparents, Reine, Della, and Albert, who taught me how to instill my soul into everything I do, how to persist in spite of insurmountable mountains, and most importantly, how to love. 


\section{Acknowledgements}

It would have been impossible to complete this project, with all of the seemingly endless obstacles, without the support of so many people. I want to first express my sincere gratitude to the chair of my committee, Dr. Margaret Glenn, who stepped into the role following the proposal and supported me to its finish with understanding, kindness, and encouragement. Your approach and guidance was exactly what I needed. Likewise, I greatly appreciate the support and assistance I received from my remaining committee members, Drs. Richard Walls, Lynda Birckhead Danley, James Bartee, and Ed Jacobs who contributed to the successful completion of this dissertation in many ways. I would also like to especially acknowledge Dr. Bob Marinelli who offered so much, both personally and professionally, to help me through the doctoral program and my initial dissertation woes. I will be forever grateful to you.

My deepest appreciation also goes to my friends and colleagues at the Center for Rehabilitation Synergy at SUNY at Buffalo, especially Dr. David Burganowski, Donna Stabler, and Michelle Thompson, who encouraged me and helped me through all the tough times and the logistical details that went into getting the project physically done in the midst of our hard work.

There are far too many individuals from Hobart and William Smith Colleges, Hilbert College, and the University of Wisconsin at Milwaukee to thank for their support in providing subjects for the study, but I would like to highlight the special assistance I received from Drs. Beth Wilson, Chip Capraro, Laura Owens, and Donna Levin. I remain grateful to all of you and amazed at the extent of your willingness to support me.

I want to offer a sincere thank you to my family, friends, and former coworkers for their prayers, encouragement, and patience throughout the years. In particular, I am deeply grateful to my children, Abigail and Zachary, who sacrificed without knowing it and inspired me in ways impossible to describe.

With all of the support I have received during this process, it is hard to imagine that any one person would stand out. However, my appreciation for all that Wendy Quarles did to help me see my way to the end can never be fully expressed. It brings such joy to my heart to know that we can finally walk through this field of gold. 


\section{TABLE OF CONTENTS}

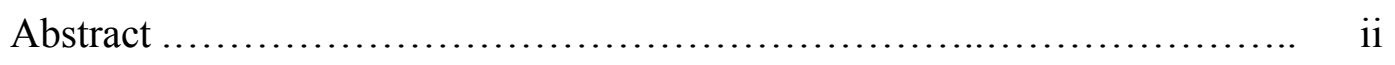

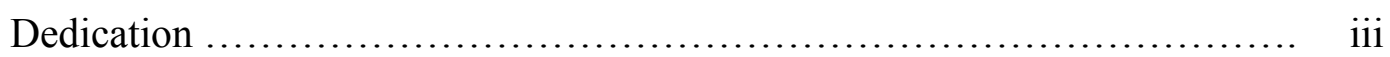

Acknowledgements .................................................... iv

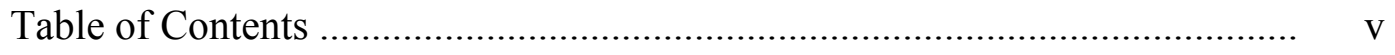

List of Tables ............................................................. ix

Chapter 1 - Introduction ..........................................................................

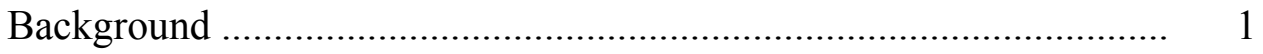

College Binge Drinking and Its Consequences ……………………..... 5

Alcohol Prevention on College Campuses ……………......................... 8

Emotional Factors Associated with Binge Drinking Consequences ..... 15

Statement of Problem …………………………………………..... 22

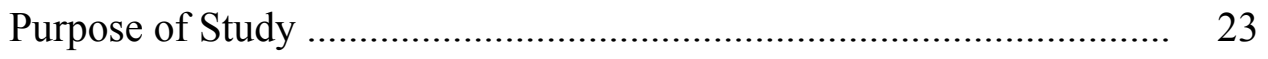

Definition of Key Concepts …………………………………......... 25

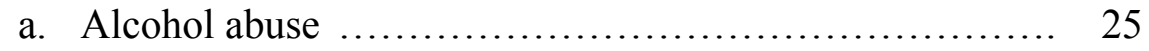

b. Alcohol-related consequences ......................... 26

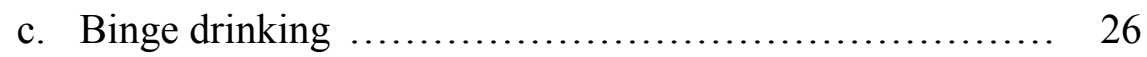

d. Emotional intelligence .............................. 27

Chapter 2 - Literature Review ………………………............................... 28

Binge Drinking Trends, Patterns and Associated Psychological Factors 28

a. Gender differences ..................................................... $\quad 33$ 
b. Ethnic differences .................................. 38

c. Other demographic factors ........................... 39

The Nature of Binge Drinking Consequences.................................. 41

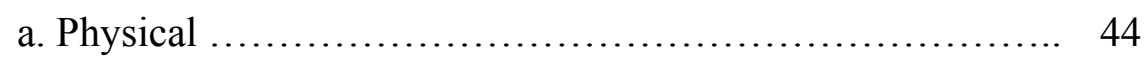

b. Social responsibility ............................... 45

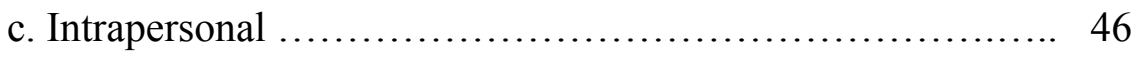

d. Impulse control $\ldots \ldots \ldots \ldots \ldots \ldots \ldots \ldots \ldots \ldots \ldots \ldots \ldots \ldots \ldots, 47$

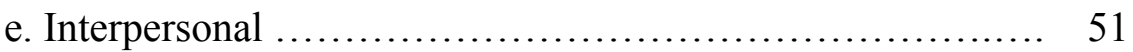

f. Consequences over the college years .................. 52

g. Demographic differences ............................................ 54

h. Other factors involved in alcohol-related consequences ...... 58

Binge Drinking as a Psychological and Interpersonal Tool ................. 62

a. Facilitation of interpersonal relations ................. 65

b. Affect management ............................... 69

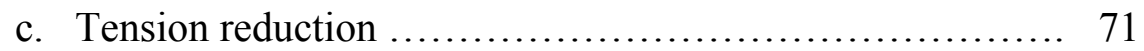

d. Avoidance coping .................................... 74

e. Gender issues ..................................... 75

f. Conclusions about college drinking ................... 78

Emotional Functioning, Behavior and Alcohol ............................. 79

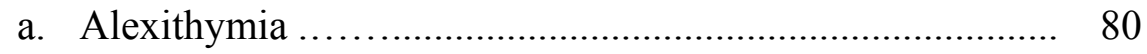

b. Emotional intelligence as a construct $\ldots \ldots \ldots \ldots \ldots \ldots \ldots . . \ldots 3$ 
c. Consequences of low emotional intelligence .................. 88

Summary and Research Questions ............................................. 90

Chapter 3 - Method ........................................................................... 95

Participants ......................................................................... 95

Measures......................................................................... 96

a. Binge drinking measures ............................................ 96

b. Alcohol-related consequence measures ................ 103

c. Emotional intelligence measures f................................ 107

Procedure ....................................................................... 114

Statistical Analyses ................................................................ 115

Chapter 4 - Results ........................................................................ 120

Preliminary Analyses ......................................................... 121

a. Demographic differences in emotional intelligence ........... 122

b. Demographic differences in binge drinking frequencies ... 124

c. Demographic differences in binge drinking consequences . 126

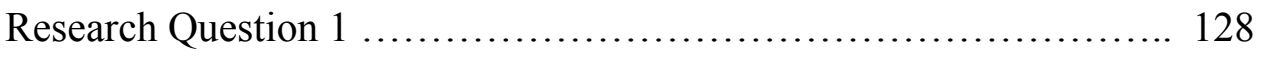

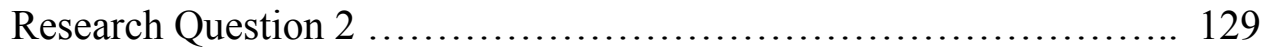

Research Question 3 ...................................... 131

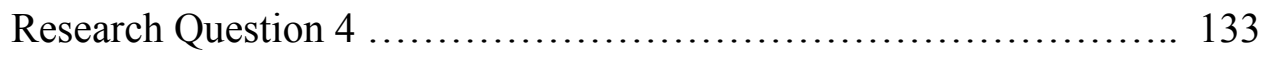

Chapter 5 - Discussion ........................................ 137

College Student Binge Drinking and Emotional Intelligence ......... 137 
Using EI to Understand Binge Drinking Consequences .............. 141

Conclusions $/$ Implications $\ldots \ldots \ldots \ldots \ldots \ldots \ldots \ldots \ldots \ldots \ldots \ldots \ldots \ldots, 148$

Limitations of the Study .................................. 153

Recommendations for Further Research ...................... 155

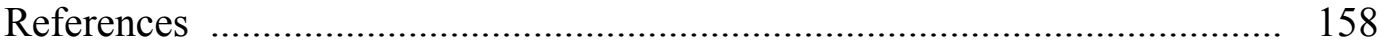

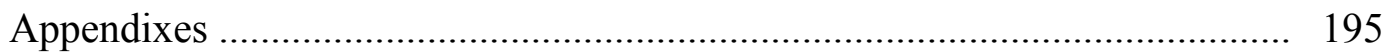

Appendix A: DSM-IV-TR Criteria for Alcohol Abuse .................. 196

Appendix B: Research Questionnaire ........................ 198

Appendix C: Examples of the Psychometric Assessments .............. 200

Appendix D: Tables ...................................... 206

Appendix E: Consent and Information Form $\ldots \ldots \ldots \ldots \ldots \ldots \ldots \ldots 243$

Curriculum Vitae ............................................ 246 
Application of Emotional Intelligence ix

\section{LIST OF TABLES}

Table 1 Reliability and Validity of the Drinker Inventory of Consequences (DrInc)

Table 2 Reliability and Validity of the BarOn Emotional Quotient Inventory (EQ-i)

Table 3 Demographic Frequencies and Percentages of Participants $(N=309) 209$

Table 4 EQ-i Means, Standard Deviations, Standard Error Scores, and Ranges for Age

Table 5 EQ-i Means, Standard Deviations, Standard Error Scores, and Ranges for Athlete Status

Table 6 EQ-i Means, Standard Deviations, Standard Error Scores, and Ranges for Class

Table 7 EQ-i Means, Standard Deviations, Standard Error Scores, and Ranges for Gender

Table 8 EQ-i Means, Standard Deviations, Standard Error Scores, and Ranges for Greek System Membership

Table 9 Binge Drinker Sample Sizes, Means, Standard Deviations, and Standard Error Scores for Binge Drinking Frequency by Age ....... 216

Table 10 Binge Drinker Sample Sizes, Means, Standard Deviations, and Standard Error Scores for Binge Drinking Frequency by Athlete Status

Table 11 Binge Drinker Sample Sizes, Means, Standard Deviations, and Standard Error Scores for Binge Drinking Frequency by Class

Table 12 Binge Drinker Sample Sizes, Means, Standard Deviations, and Standard Error Scores for Binge Drinking Frequency by Gender .... 219

Table 13 Binge Drinker Sample Sizes, Means, Standard Deviations, and Standard Error Scores for Binge Drinking Frequency by Greek Membership 
Table 14 Binge Drinking Consequence (DrInc Total Scores) Means, Standard Deviations, Standard Error Scores, and Ranges for Age .. 221

Table 15 Binge Drinking Consequence (DrInc Total Scores) Means, Standard Deviations, Standard Error Scores, and Ranges for Athlete Status

Table 16 Binge Drinking Consequence (DrInc Total Scores) Means, Standard Deviations, Standard Error Scores, and Ranges for Class.. 223

Table 17 Binge Drinking Consequence (DrInc Total Scores) Means, Standard Deviations, Standard Error Scores, and Ranges for Gender 224

Table 18 Binge Drinking Consequence (DrInc Total Scores) Means, Standard Deviations, Standard Error Scores, and Ranges for Greek Membership

Table 19 Summary Tables of the Statistical Analyses for Differences in Binge Drinking Consequences within Age 226

Table 20 Summary Tables of the Statistical Analyses for Differences in Binge Drinking Consequences within Class Year

Table 21 Summary of the Stepwise Regression Analysis for EI Variables Predicting Physical Binge Drinking Consequences 228

Table 22 ANOVA for the Regression Analysis Model Predicting Physical Consequences

Table 23 Coefficients for the Regression Analysis Model Predicting Physical Consequences

Table 24 Summary of the Stepwise Regression Analysis for EI Variables Predicting Intrapersonal Binge Drinking Consequences

Table 25 ANOVA for the Regression Analysis Model Predicting Intrapersonal Consequences

Table 26 Coefficients for the Regression Analysis Model Predicting Intrapersonal Consequences 
Table 27 Summary of the Stepwise Regression Analysis for EI Variables Predicting Interpersonal Binge Drinking Consequences

Table 28 ANOVA for the Regression Analysis Model Predicting Interpersonal Consequences ................................... 235

Table 29 Coefficients for the Regression Analysis Model Predicting Interpersonal Consequences .................................... 236

Table 30 Summary of the Stepwise Regression Analysis for EI Variables Predicting Impulse Control Binge Drinking Consequences ........ 237

Table 31 ANOVA for the Regression Analysis Model Predicting Impulse Control Consequences ....................................... 238

Table 32 Coefficients for the Regression Analysis Model Predicting Impulse Control Consequences .......................................... 239

Table 33 Summary of the Stepwise Regression Analysis for EI Variables Predicting Social Responsibility Binge Drinking Consequences .... 240

Table 34 ANOVA for the Regression Analysis Model Predicting Social Responsibility Consequences ................................. 241

Table 35 Coefficients for the Regression Analysis Model Predicting Social Responsibility Consequences ................................. 242 


\title{
CHAPTER ONE
}

\author{
Introduction
}

\section{Background}

The mounting number of deaths, injuries, and damage due to excessive alcohol use has been a dominant concern of our society and, in particular, our nation's colleges. The negative impact of alcohol abuse is severe and persists despite the efforts of alcohol prevention programs. The phrase "binge drinking" has been coined to describe a level of alcohol consumption in which there is a significant increase in related consequences; this level has been identified as four or more drinks for females and five or more drinks for males on one "drinking" occasion within a two week period (Wechsler, Davenport, Dowdall, Moeykins, \& Castillo, 1994; Ziemelis, Bucknam, \& Abdulaziz, 2002).

Recent news articles in the media have continued to address the grave and persistent nature of binge drinking on college campuses. In an article for USA Today, Marklein (2002) reported on five alcohol-related deaths on college campuses within one month. CNN.com (“Study: College drinking," 2002) posted an article in which alcohol was implicated in approximately 1,400 college student deaths per year and in 70,000 cases of sexual assault. The statistics are alarming, but equally astonishing is the lack of significant impact that the numerous research studies and alcohol prevention programs appear to have on resolving the 
problem (Walters \& Bennett, 2000; Wechsler, Dowdall, Maenner, Gledhill-Hoyt \& Lee, 1998).

Binge drinking and its consequences for college students are not going away easily, and students continue to drink at dangerous levels despite their awareness of the potential problems that could arise. One can assume that the vast majority of students don't drink alcohol to experience problems in life. The question as to why students are willing to put themselves at such great risk has led to the understanding that binge drinking is a complicated behavior motivated by multiple factors (National Institute on Alcohol Abuse and Alcoholism [NIAAA], 1997; Wechsler, Lee, Kuo, et al., 2002).

Due to the complex nature of binge drinking and its "cure" being somewhat of an enigma, researchers from many areas have attempted to offer some insight. Leonard and Blane (1999a) compiled the latest research and theories on drinking and alcoholism from the fields of neurobiology, genetics, personality and human development, social learning, learning and cognition, and emotion and tension reduction. Their conclusion acknowledged the lack of one "answer" to the problem but accepted that progress has been made with regard to alcohol treatment. Further integration of research that would allow alcohol problems to be viewed as a multivariate construct was encouraged to enhance the development of alcohol prevention programs and public policy (Leonard \& Blane, 1999b). 
The phenomenon of binge drinking has multiple dimensions which suggests that several directions for alcohol prevention programs should be considered in order to confront different aspects and levels of the problem (Graczyk et al., 2001). Colleges and universities have made a number of attempts at reducing binge drinking using a variety of direct prevention models and policy changes with some limited success (Ziemelis et al., 2002). However, the reduction of binge drinking does not appear to coincide with the decrease in alcohol-related consequences on campuses (Wechsler, Lee, Kuo, et al., 2002). Therefore, the consequences of binge drinking may need to be addressed through prevention programs that go beyond confronting alcohol use directly. It may be valuable to develop prevention models that also target other factors associated with these consequences, such as affect management and the ability to effectively negotiate interpersonal relations.

Lang, Patrick, and Stritzke (1999) state that alcohol, like other psychoactive substances, is chemically designed to be mood altering and to cause changes in consciousness. As a result, some emotional and sociocultural theories of alcohol use propose that the physical nature of alcohol could lead to disinhibition and the perceived freedom from social constraints. The authors note that the potential to have behavioral consequences following alcohol use may arise from these changes in mood and consciousness that encourage disinhibition. However, in cultures all over the world, the effects of alcohol are also strongly 
tied to socialization with both positive and desired effects, such as with community celebrations and religious ceremonies. Thus, the "freedoms" experienced by alcohol use can have both social benefits and disastrous consequences depending on the individual, culture and context of the drinking. A potential conflict is obviously present for those college students who may seek alcohol to facilitate social connections, manage mood states, or aid the development of meaningful relationships.

Alcohol's effects on mood, consciousness, and socialization can lead to various outcomes which implies that the pharmacological components of alcohol alone do not directly cause specific behavior changes, such as increased aggression (Bushman \& Cooper, 1990). The contribution of individual differences must be taken into account when attempting to understand the nature of consequences or when developing prevention models. There has been substantial theory and research on the demographic differences and multiple paths of alcoholism and binge drinking (Leonard \& Blane, 1999a). However, relatively few studies have been conducted on the individual differences that contribute to the negative consequences of binge drinking. How individuals differ in managing $\operatorname{mood} /$ affect and interpersonal relations could have strong implications for how they respond to disinhibiting effects of alcohol (Turrisi, Wiersma, \& Hughes, 2000). 
The present study will examine the ability of college students to manage affect and interpersonal relationships and how differences in these abilities may be associated to the negative consequences of binge drinking. By focusing on the psychological factors that relate to these consequences, prevention efforts may be improved by incorporating targeted wellness programs that encourage healthy affect management and interpersonal skill building. Facilitating the emotional growth of college students could yield the desired reduction in the severe costs of binge drinking as students begin to make healthier choices.

\section{College Binge Drinking and Its Consequences}

The scope of the costs that occur from college binge drinking is expansive, the consequences are severe, and the number of college students involved is high. Recent statistics reveal that approximately $45 \%$ of college students are binge drinkers, and since 1993, the number of frequent binge drinkers has been increasing as the overall number of binge drinkers remains steady (Wechsler, Lee, Kuo, et al., 2002). Furthermore, Knight et al. (2002) noted that $31 \%$ of college students fit the diagnostic criteria for alcohol abuse.

No one demographic group of college students is immune from binge drinking, although some differences do occur. College men (48.6\%) are more likely than college women (40.9\%) to engage in binge drinking (Humara \& Sherman, 1999; Wechsler, Lee, Kuo, et al., 2002) and experience negative consequences, but the rates of young adults starting to binge drink in college 
increase faster for women than men (Berkowitz \& Perkins, 1987). In terms of racial and cultural differences, Asian-American (26.2\%) students have had the greatest increase in binge drinking since 1993 (Wechsler et al., 1998), but significant decreases have been recently found among Hispanic (34.4\%) and Native American (33.6\%) college students (Wechsler, Lee, Kuo, et. al, 2002). Caucasians (50.2\%), who have the highest rates, and African-American (21.7\%) students both showed an increase since 1993, but the differences were not significant (Wechsler, Lee, Kuo, et. al, 2002). Although gay and bisexual men (29\%) have lower heavy drinking than heterosexual men (48\%), gay, lesbian and bisexual adolescents and college students are more likely to be at risk and have drinking and other drug problems than their heterosexual peers (Caldwell, Kivel, Smith, \& Hayes, 1998; Debord, Wood, Sher, \& Good, 1998; McCabe, Boyd, Hughes, \& d'Arcy, 2003; Ziyadh et al., 2007).

Binge drinking on an occasional or frequent basis has become a pervasive problem that appears to be associated with few, if any, deterrents. Aside from the direct consequences of binge drinking, factors that would be expected to inhibit drinking severity seem to have no impact. The binge drinking levels of college students are not typically found in the families of the students (Rabow \& Neuman, 1984) or in their non-college peers (Quigley \& Marlatt, 1996). Surprisingly, the phenomenon of binge drinking remains centered on young college students who 
choose to drink in excess despite the awareness of the mounting evidence of problems and negative consequences associated to it.

Vik, Carrello, Tate, and Field (2000) surveyed college students at a major university and found that for nine of ten binge drinkers, alcohol intoxication was blamed for some form of careless behavior, such as academic problems (i.e. decreased class attendance or missed/late assignments), interpersonal violence and conflict, physical injury and blackouts, or property damage. Almost twothirds of binge drinking students put themselves at great risk for severe consequences with such actions as driving while intoxicated or unprotected or unplanned sexual behavior. Legal or academic disciplinary action was experienced by one-third of the student binge drinkers. Fisher, Sloan, Cullen, and Lu (1998) reported that alcohol plays a significant role in crime on college campuses.

In terms of the potential risks posed to an individual's health, binge drinking has been linked to cognitive and neurological impairment (Altura, Gebrewold, Zhang, Altura, \& Gupta, 1998; Browning, Hoffer, \& Dunwiddie, 1992; Hunt, 1993), cigarette smoking (Emmons, Wechsler, Dowdall, \& Abraham, 1998), and illicit substance use (Bell, Wechsler, \& Johnston, 1997). Interestingly, college student athletes, who would likely be concerned with their physical health, experience more alcohol-related consequences than non-student athletes (Leichliter, Meilman, Presley, \& Cashin, 1998). The association between binge 
drinking and negative consequences is high and visible to most binge drinkers, yet the behavior continues regardless of the obvious personal and interpersonal pain and the alcohol prevention efforts on college campuses. Further study is necessary to understand the psychological factors that could have implications for the development of enhanced approaches to the reduction and prevention of binge drinking consequences.

\section{Alcohol Prevention on College Campuses}

College administrators who work at schools with high levels of drinking encounter a number of persistent behavioral problems on their campuses. One survey indicated that sexual assault was a significant problem for $86 \%$ of these administrators; $61 \%$ noted frequent episodes of physical assault; and $53 \%$ had to deal with damage to campus property on a recurring basis (Wechsler, Moeykens, Davenport, Castillo, \& Hansen, 1995). In an effort to combat the high levels of alcohol-related problems, prevention programs have been developed to reduce alcohol consumption. The assumption is that education could lower alcohol use on campus and therefore, its consequences. Wechsler, Kelley, Weitzman, SanGiovanni, and Seibring (2000) reported that among 734 college administrators polled, 97\% implement an alcohol prevention program that involves some form of education. Most administrators also attempt to modify the campus environment by restricting alcohol supply and advertising and by offering alcohol-free dormitories. 
The effort of colleges to limit alcohol use can hardly be questioned, yet the prevention programs fail to yield the desired results. Wechsler et al. (1998) surveyed 116 colleges and found that only a small reduction in binge drinking rates occurred since 1993, but alcohol-related problems were overall on the rise. Research on prevention has consistently shown that a focus primarily on alcohol education has no impact on reducing binge drinking on college campuses (Walters \& Bennett, 2000). Schulenberg et al. (2001) cited several studies that collectively demonstrate the difficulty prevention programs are having achieving significant results. Although a few programs were able to show some effect, the authors could not definitively say that there are clear approaches to prevention beyond direct alcohol education.

Other studies on the effectiveness of college binge drinking prevention programs have been inconclusive. Murgraff, White, and Phillips (1996) were able to decrease alcohol frequency among undergraduates two weeks after the students attended a prevention program. The curriculum included information on binge drinking risks and consequences and instruction on how to limit their alcohol intake. No evidence was reported that the intervention decreased alcohol-related consequences or that the changes were maintained beyond two weeks. Knight et al. (2002) examined colleges who have instituted an alcohol-free policy on their campus and alcohol-free dormitories. The rates of students who met the criteria for alcohol abuse or dependence were not lower if they attended a college with an 
alcohol-free campus. The only positive difference was observed when students lived in alcohol-free dormitories. Students in these living situations had a lower risk of abuse but dependence remained the same across dormitories.

An innovative approach to prevention has been the use of social norms programming in which there is a dissemination of positive, factual alcohol and health related information across campus (Haines \& Spear, 1996). The idea is based on two research findings which suggest that for college students, the level of alcohol use is influenced by how students perceive the drinking habits of their peer group and that this perception of the peer group's drinking tends to be overestimated (Baer, Stacy, \& Larimer, 1991). By correcting these misperceptions of the "norm" and by promoting healthier facts representative of their peers, a positive change in student behavior is expected.

Wechsler and Kuo (2000) cited some research support for this hypothesis and took a closer look at the statistics surrounding the perceived drinking norms on college campuses. More than half of the students overestimate how many drinks constitute binge drinking. As a result, approximately $47 \%$ of the sample thought that the binge drinking rates were lower than what actually occurs, perceiving that only $35 \%$ of the students binge drink at their respective schools. More importantly, an overestimation of the binge drinking rate was found in only $13 \%$ of the entire sample and in $29 \%$ of binge drinkers. The authors hypothesized that it is this $13 \%$ of the college student population who may have the desired 
response to the social norms approach. Recently, Neighbors, Lee, Lewis, Fossos, and Larimer (2007) found that excessive alcohol consumption among college students could be significantly predicted by the social drinking norms of the campus. However, drinking with the intention to use alcohol to cope with stress was a better predictor of alcohol-related problems.

Other prevention programs put significant emphasis on the campus and community environment. Laws, fines, college disciplinary protocols, alcohol availability, and recreational/social alternatives have been modified to inhibit alcohol use. Bishop (2000) implemented a comprehensive environmental approach to prevention that involved college policy changes, alternative social opportunities without alcohol, and increased efforts from the community outside of campus. The combination of heightened restrictions on alcohol use, improved social options, increased penalties for alcohol violations, and related factors were associated with some reduced alcohol-related consequences on campus, but changes to binge drinking patterns were not measured.

As a deterrent to underage drinking, Wechsler, Lee, Nelson, and Kuo (2002) found that states which have more severe legal restrictions on alcohol use tend to have less binge drinking and overall alcohol consumption among underage students. The downside is that underage students tend to drink less frequently than their "legal-aged" peers but with higher quantities per occasion. Also, approximately half of the underage students who seek alcohol are able to find 
opportunities to drink without much difficulty. Legal/disciplinary penalties and alcohol restrictions may have some impact on the reduction of binge drinking, but this form of prevention doesn't address the fact that many well-educated, young adults knowingly put themselves at risk for a destructive pattern of alcohol use. An environmental approach may have a limited effect on reducing alcohol-related consequences if the problems are found to reflect a student's psychological or developmental state more than the intoxicating effects of alcohol use.

Goldman (1994) proposed that traditional alcohol prevention programs could have difficulty achieving their goals as they face the normal developmental challenges of young adults and their positive perception of alcohol as an antidote to these struggles. If a student's level of cognitive and emotional development does significantly contribute to binge drinking, prevention efforts need to consider what is happening within the individual in addition to providing alcohol education, reducing easy access to alcohol, and modifying perceptions of peer drinking. Furthermore, by adding a focus on improving the emotional and interpersonal well-being of a student, a broader range of problems and factors may be addressed that could have an indirect contribution to binge drinking and its consequences (Graczyk et al., 2001).

Hawkins and Catalano (1992) surveyed alcohol and other drug prevention programs that report positive results. Some frequently included elements to these programs are emotional skill building (i.e., identifying and labeling feelings, 
expressing feelings, assessing the intensity of feelings, managing feelings, delaying gratification, controlling impulses, reducing stress, knowing the difference between feelings and actions), understanding the perspective of others, a positive attitude toward life, and self-awareness. A study of 48 community prevention programs targeting youth substance abuse also showed a pattern of success across programs emphasizing improved interpersonal relations, life skills and self-examination (Smith, 2002). Girls appeared to benefit more from lifeskills development, and boys did best with programs that offered interpersonal skill building.

A national survey of college alcohol prevention programs between 1993 and 2001 revealed that there is no one prevention format that confronts the alcohol problem in a framework suitable for all colleges (Wechsler, Lee, Kuo et al., 2002). With no single, clear solution, binge drinking reduction is now being addressed at some institutions by a multimodal prevention approach that is comprehensive, dynamic, and beneficial to students' overall emotional development. Ziemelis et al. (2002) compiled the results of a survey of alcohol and other drug prevention programs from 94 campuses and derived 3 effective prevention strategies from the data. One strategy is to increase student involvement in committees or other groups that have decision making abilities on alcohol and other drug issues on campus. The authors believe that improving students' sense of agency and value for solving the alcohol problem could be 
beneficial. Secondly, modifying the socio/cultural environment of the campus, via displays, resource centers, and curriculum infusion, also appears to have some positive effect. One stipulation is that the students need to feel that the information is direct, the process collaborative, and the administrators and educators not condescending. A third strategy is to change and enforce the campus policies on alcohol and other drug use, have mandatory prevention classes, and add social activities without alcohol. Although the researchers did not focus on non-alcohol related programs, health and wellness education seminars were associated with significantly more campuses that demonstrated a reduction in binge drinking.

Another aspect of a multimodal approach to prevent binge drinking has been to offer a variety of programs to address different levels of the problem severity. Kim, Larimer, Walker, and Marlatt (1997) proposed alternative prevention programs that would be based on a continuum of seriousness of the binge drinking offense. Students with alcohol violations would be sent to specific educational programs in which the curricula would reflect the extent of the alcohol problem. Wellness programs were considered the earliest stage of prevention. With increasing numbers of alcohol violations and/or consequences, the prevention program would involve a more extensive and direct approach.

Research studies on alcohol prevention programs reveal the need for considerably more information on the roots of binge drinking and on how to 
reduce the devastating consequences which seem to persist even when drinking rates have decreased. Some programs do appear to have had an impact on binge drinking but not to the extent that allows confidence in any one approach. Wellness programs and education seminars that incorporate affect management and interpersonal relationship skill building have shown promising results, but the focus of these programs has not yet been fully defined. Customizing wellness programs to suit the emotional or interpersonal nature of a frequent binge drinking consequence could provide multiple benefits to the student and the campus community. For example, binge drinking consequences could reduce while interpersonal relationships improve. Before this can be achieved, the consequences of binge drinking need to be broken down in useful terms to aid in the design of targeted wellness prevention programs.

Emotional Factors Associated with Binge Drinking Consequences

Young adults in the college setting encounter numerous stressors on a daily basis, beginning with the transition to college and the loss of many of the social supports from their high school years, especially family. The energy it takes to sustain oneself emotionally and socially during this period can tax an individual's ability to cope with stress and to manage uncomfortable affect. It is this transitional period of a student's life that researchers find a significant increase in binge drinking and problems associated to alcohol (Schulenberg \& Maggs, 2002). This observation leads to a concern about the potential heightened 
negative effect that alcohol and high stress could have on students who come to college emotionally and interpersonally immature. One model proposes that those students who start with difficulties managing the psychological aspects of their life (i.e., transitions) will grow increasingly distant from their well-functioning peers and be more susceptible to alcohol problems (Schulenberg \& Maggs, 2002). Thus, understanding differences in binge drinking consequences may require an understanding of differences among students and within a student over time.

Baer (2002) reviewed the literature on individual differences and alcohol use and found that there are a variety of motivating psychological factors and contexts, beyond personality characteristics, that contribute to alcohol use. For example, the research suggests that differences in the sociability of college students (i.e., introversion, extraversion, intimacy, "party seeking”) correspond with the diversity in student drinking patterns more than differences in other personality traits (i.e., impulsivity, self-esteem). One qualification of this summarization is that those who tend to drink to manage their affect are more likely to experience negative consequences than those who drink to enhance their socialization. Both increased alcohol intake and alcohol-related problems are related to impulsivity/sensation-seeking type drinking, stress/anxiety-based drinking, and the overall presence of negative emotional states. Likewise, college students with anxiety disorders are also more likely to be diagnosed with alcohol abuse or dependence. Understanding the emotional and interpersonal factors 
involved in binge drinking may be critical for breaking down the complexity of alcohol problems. Differences among college students on these dimensions need to be examined in greater detail in order to determine what forms of wellness programs may work best in alcohol prevention.

One emotional factor that has been studied more closely in terms of its relationship with alcohol problems is aggression. An overview of the literature on alcohol-related aggression among college students strongly supports the influence that alcohol has on aggression along with the increased risk for heavy drinkers to become victims of violence themselves (Giancola, 2002). A clear example of alcohol-related aggression was demonstrated by Taylor and Sears (1988). The performances of sober and intoxicated subjects were compared on the Taylor Aggression Paradigm (TAP) after a confederate had attempted to persuade them to be aggressive. Only intoxicated subjects were vulnerable to the persuasion and exhibited aggression towards an opponent. However, a closer look at alcohol and aggression reveals the need to again consider individual differences. Bailey and Taylor (1991) also administered the TAP to study alcohol use and its effects on college students with dispositional aggression. Men who were determined to be aggressive by disposition had a significant increase in aggression upon alcohol intoxication as compared to no increase for men with low to moderate dispositional aggression. 
In addition to the finding that alcohol increases aggression for those predisposed to aggression, there is the belief that individuals with lower executive functioning may also be more susceptible to alcohol's influence. Executive functioning is defined as the ability to control those mental functions that direct and regulate cognitive and social behavior, such as actively paying attention, intentional recall of memories and being polite to strangers (Ylvisaker, Szekeres, \& Feeney, 1998). Alcohol is thought by some theorists to compound the difficulty individuals with poor executive functioning have enacting healthy, nonaggressive behaviors as an alternative to aggression (Giancola, 2000). The hypothesis is if a situation provokes an aggressive response, aggression would be the likely outcome when the cognitive ability to regulate behavior is hindered by low executive functioning and the impairing effects of alcohol. Lau, Pihl, and Peteron (1995) studied the relationship among alcohol use, executive functioning, and aggression. The TAP was used to examine how alcohol might contribute to aggression for male college students with low and high executive functioning (assessed by neuropsychological tests). Contrary to the above hypothesis, no interaction effect between executive functioning and alcohol was found, but alcohol and low executive functioning had separate effects on aggression. The data suggest that alcohol may affect an individual's ability to manage aggression in a way that is not explained by their cognitive potential. An alternative hypothesis is that alcohol has an impairing effect on an individual's ability to 
manage affect and interpersonal relationships as she or he experiences a provocative situation. In other words, it may be that emotional, rather than intellectual, deficits make one more vulnerable to the negative effects of alcohol.

Giancola (2002) compiled the research findings on aggression and alcohol, and among the list of factors connected with alcohol-related aggression were: perspective-taking, self-awareness, negative affect, temperament, affect regulation, emotionality, sensation seeking, anxiety, irritability, hostility, frustration tolerance, impulsivity, and perceived self-esteem. Based on the multiple emotional factors involved, the reduction of alcohol-related aggression will not be achieved by targeting prevention programs toward one direction or type of college student. The author suggested that prevention efforts encompass several areas of alcohol and psychological instruction, including psychological harm reduction strategies and information that helps students improve their social functioning and overall interpersonal skills.

Aggressive behavior is only one form of consequence that can occur from binge drinking, and other emotional deficits and variables appear to play key roles in its expression. By understanding the emotional functioning of students who have certain alcohol-related consequences, valuable information may be gathered to help these students by implementing wellness programs designed to inhibit these emotional problems from occurring in both sober and intoxicated states. 
Goleman (1995) stated that the very definition of emotion involves a drive to action that is derived from a feeling. The outcome or consequence of the action depends on the individual and the context in which the feeling is experienced. Gardner (1983) proposed that just as individuals differ in terms of cognitive intelligence, they may also differ on other types of intelligences that can have a significant impact on how an individual functions in life. One type is intrapersonal intelligence which is described as an individual's capacity to manage their own emotional life. The other, interpersonal intelligence, involves the potential of an individual to comprehend the emotional expression and context of another and relate to him or her empathically.

Recently, a construct called emotional intelligence (EI) has been used to encapsulate and expand on the two types of intelligences proposed by Gardner. EI has been defined as "the capacity to reason with emotion in four areas: to perceive emotion, to integrate it in thought, to understand it and to manage it" (Mayer, 1999, pp.1-2). The ability to address emotion in these ways takes place both intrapersonally (within the individual) and interpersonally (Bar-On, 1997/2002). Students who begin their college career with low EI could be at a significant disadvantage if they have difficulty functioning in the emotional and interpersonal areas of campus life. Furthermore, it is not yet known how a student with low EI could be vulnerable to the effects of alcohol when it enters the student's environment. 
Previous studies have shown that deficits in EI and several its components are connected to alcohol use and related problems (Baer, 2002; Giancola, 2002; Riley \& Schutte, 2003; Trinidad \& Johnson, 2002). Likewise, evidence has been cited above to demonstrate how some effective alcohol prevention models incorporate wellness programs with instruction on affect management and interpersonal skill building. Additional research is needed to determine if EI can be a useful construct for understanding binge drinking and its consequences. Breaking down EI and alcohol-related consequences into separate factors may also yield beneficial details for designing specific wellness programs for identified audiences.

Recommendations have been made to define alcohol problems as a heterogeneous variable in future alcohol research that could lead several possible paths of treatment and prevention (Institute of Medicine, 1990). Vik et al. (2000) moved in the direction of breaking down alcohol problems by separating the problems by degree of seriousness. The three problem domains were: careless behavior, risky/reckless behavior, and authority problems. These domains were then studied in relation to personality factors to discern any correlations or trends that occur. A few differences in personality were found across the problem domains. However, many other valuable relationships may have been obtained if alcohol-related consequences were separated by psychological factors (i.e., intrapersonal vs. interpersonal consequences). 


\section{Statement of Problem}

Numerous articles from the media and research literature have reported on the vast costs of college binge drinking to the students who drink, their peers, college campuses, and the surrounding communities. To combat the problem, alcohol prevention programs have traditionally focused on restricting access to alcohol and educating students on the consequences of binge drinking. In general, these efforts have not significantly reduced binge drinking among college students, and consequences continue to rise. There have been some promising results from prevention and wellness programs that encourage healthy affect management and interpersonal skill building, but more research is needed to understand which components are critical to reducing alcohol-related consequences. In addition, there may be sub-populations of college binge drinkers who would respond best to different types of information and education.

To aid in the development of these customized prevention programs, colleges need to be aware of the individual differences that may be involved in such a complex issue as binge drinking. Research studies have consistently shown a connection between emotional factors and alcohol, especially alcohol-related aggression. It is clear from these studies that increased alcohol intake is related to a variety of behavioral and emotional outcomes depending on the student. Some students may have deficits in EI and possess an impaired ability to understand and cope with one's own feelings and/or to negotiate interpersonal relations. If 
students are relying on the desired effects of alcohol to overcome these deficits of EI, the opportunities for negative consequences could increase. There may be occasions when the disinhibiting effect of alcohol within a provocative context exacerbates a deficit, and a hurtful or regretful decision is made (i.e., to keep drinking, to fight, to drive, or to be sexually active). This notion that emotional and interpersonal deficits could relate to specific types of binge drinking consequences has not yet been examined.

Binge drinking among college students is a complex problem that demands a comprehensive prevention approach. Colleges will need to avoid the temptation to use one program to prevent binge drinking and consider targeting multiple levels of the problem. To facilitate the development of customized wellness prevention programs, the present study will explore how deficits in the various components of EI relate to different types of alcohol-related consequences.

Purpose of Study

Although extensive research has been conducted in the area of college binge drinking, relatively little has been done to study differences among alcoholrelated consequences. If the consequences of binge drinking are not random, alcohol prevention programs can be designed to address the possible source(s) of specific problem areas. The EI construct has been growing in terms of popularity and credibility, yet it has been rarely studied in conjunction with alcohol. 
Understanding this relationship could be valuable due to the association of several emotional and interpersonal factors to binge drinking and its consequences. The present study attempts to discern what relationships exist among EI factors and the different types of binge drinking consequences. The results could lead to an expansion of the theoretical considerations for alcohol-related consequences. Furthermore, alcohol prevention programs could incorporate, as part of a multimodal approach, specific wellness training based on the type of consequence.

If binge drinking consequences are shown to be indicative of emotional and interpersonal deficits, improving these deficits could also have benefits beyond the reduction of alcohol problems, such as healthier relationships with peers, administration, and faculty. Lastly, students who may be resistant to lowering their alcohol consumption may be less defensive around programming that they perceive to be more helpful than confrontational, as in the case of direct alcohol education.

The following research questions, derived from the literature on college student binge drinking, alcohol abuse and EI, are proposed for the present study:

1) Do college binge drinkers and non-binge drinkers differ on a measure of emotional intelligence (EI)?

2) Do binge drinkers with consequences differ from binge drinkers without consequences on a measure of EI? 
3) Are there emotional and interpersonal components of EI that predict different types of alcohol-related consequences across binge drinking college students?

4) If significant relationships exist among EI factors and specific types of alcohol-related consequences, are there any variations across demographic variables?

\section{Definition of Key Concepts}

Alcohol abuse. If a level of alcohol consumption is present in which the direct intoxication or withdrawal effects of alcohol lead to impairments in academic or job performance, alcohol abuse would be indicated. In addition, the diagnosis is warranted if these effects also lead to increased risk of harm to self or others or to legal problems. Another component of alcohol abuse is the maintenance or increase of the rate of alcohol consumption despite the individual's awareness of the consequences of his or her alcohol use. Alcohol abuse is distinguished from alcohol dependency by the lack of tolerance, severe withdrawal effects, or compulsive behavior related to alcohol use. Binge drinking is considered one form of alcohol abuse. See Appendix A for the complete diagnostic criteria according to the Diagnostic and Statistical Manual of Mental Disorders ( $4^{\text {th }}$ ed., text rev.; DSM-IV-TR; American Psychiatric Association, 2000). 
Alcohol-related consequences. The phrase "alcohol-related consequences" refers to problems that occur during or after alcohol intoxication that can be directly attributed to the effects of alcohol use. These types of alcohol problems should be distinguished from pathological drinking behavior and alcohol dependence (Miller \& Tonigan, 1995). Consequences may be present as negative personal psychological experiences, interpersonal problems, physical illness and health concerns, or impaired academic or work performance. The Drinker Inventory of Consequences (DrInC; Miller \& Tonigan, 1995) will be administered to subjects to operationally define a variety of alcohol-related consequence categories (physical, social responsibility, intrapersonal, impulse control, and interpersonal).

Binge drinking. This once subjective phrase used to describe a rapid and dangerous level of drinking now represents a specific quantity of alcohol consumed within limited time frames. Wechsler, Davenport et al. (1994) found that the number of alcohol-related social consequences increased significantly when men drank five or more drinks in a row and women drank four drinks in a row during a two week period of time. This "five/four measure of binge drinking" was used successfully to predict $92 \%$ of the students who experienced five or more alcohol related problems. Ziemelis et al. (2002) endorsed this measure of binge drinking as a valid indicator of high-risk, alcohol-related problem behavior. Frequent binge drinking is considered three or more binge drinking episodes 
within the past two weeks (Wechsler et al., 1998). The "five/four measure" will be used as the operational definition of binge drinking and assessed via modified questions from the Alcohol Use Disorders Identification Test (AUDIT; Babor, de la Fuente, Saunders, \& Grant, 1992).

Emotional intelligence (EI). Due to its recent theoretical development, the components of the emotional intelligence construct have been strongly debated. A generally accepted definition of EI is "the capacity to reason with emotion in four areas: to perceive emotion, to integrate it in thought, to understand it, and to manage it" (Mayer, 1999, pp.1-2). Bar-On (1997/2002) researched EI as a multidimensional construct of noncognitive emotional abilities broken down into five measurable categories: intrapersonal (emotional awareness, assertiveness, self-regard, self-actualization, and independence), interpersonal (empathy, interpersonal relationships, social responsibility), adaptability (problem solving and reality testing), stress management (stress tolerance, impulse control), and general mood (happiness, optimism). These categories and subcategories make up the design of the BarOn Emotional Quotient-Inventory (EQ-i; Bar-On, 1997/2002) which will be used to operationally define EI and its factors. 


\section{CHAPTER TWO}

Literature Review

The study of college binge drinking has traditionally involved extensive survey research across colleges as well as empirical reports of the effectiveness of strategies to reduce drinking. The results have produced a general understanding of the subject, generated from alarming descriptive statistics, informative demographic data, and early ideas about what works and doesn't work in prevention. The efforts have also encouraged opportunities for further funding and support of research and the development of innovative alcohol prevention programs. Although survey and prevention research has been valuable to identify the scope of the problem, patterns of use and possible solutions, the literature is limited regarding the factors within the individual that may be contributing to binge drinking and its consequences. In particular, the role of emotional and interpersonal variables in college binge drinking has received some initial support, but greater detail is needed before using the data to develop more effective prevention models.

Binge Drinking Trends, Patterns, and Associated Psychological Factors

Prior to undertaking any initiative to reduce college binge drinking, researchers must face the impenetrable consistency of binge drinking rates over the years. In one of the most comprehensive and cited national surveys conducted of college drinking, Wechsler, Lee, Kuo, et al. (2002) reported that $44.4 \%$ of 
college students meet the criteria for binge drinking. The rate has remained remarkably steady across three earlier surveys beginning in 1993. The same pattern was true for another series of national surveys of approximately 4000 college students representing every state. Hanson and Engs (1992) found that binge drinking rates did not significantly fluctuate across the years of 1982, 1985, 1988, and 1991. The changes that have been occurring mostly haven't been positive. Although the percentage of abstainers has been increasing since 1997, the percentage of frequent binge drinkers has also risen. Likewise, populations who have typically been immune from binge drinking, such as students from allwomen colleges, are experiencing a sharp increase in binge drinking (Wechsler, Lee, Kuo, et al., 2002).

Another perspective of the data, offered by Wechsler et al. (1998), is to consider that almost half of all college drinkers binge drink (or 2 of 5 college students) with 1 of 5 college students classified as a frequent binge drinker; another 1 of 5 are abstainers from alcohol. It should be noted that alcohol-related problems have significantly increased for all college student drinkers which includes engaging in such high-risk behavior as drinking and driving. Another important finding is that alcohol use patterns in high school were predictive of binge drinking in college, an observation supported by McCabe (2002). The consistent pattern of use for an individual across environments suggests that 
emotional and interpersonal factors unique to the individual may be key contributors to the persistence of binge drinking for some.

Weingardt et al. (1998) conducted a longitudinal study demonstrating that as students progress through their college years, consumption patterns become more dynamic with only a small segment of the college population maintaining heavy drinking, although gender differences do occur in the pattern (McCabe, 2002). Quigley and Marlatt (1996) proposed that the growing maturity of college students, combined with increasing responsibilities, encourages a reduction in abusive drinking over time. They noted that noncollege peers have lower binge drinking rates due possibly to the demands of work and marriage which took the place of a college education.

However, not every young adult appears to modify their drinking as they age or take on normal adult responsibilities. Schulenberg, O’Malley, Bachman, Wadsworth, and Johnston (1996) conducted a 9-year, longitudinal study in which they followed high school seniors and observed their binge drinking patterns as they aged. Six patterns emerged based on changes in the frequency of binge episodes and problems associated with alcohol. Those subjects who maintained a chronic pattern of frequent binge drinking or increased their rate of binge drinking tended to experience developmental difficulties as they entered into adulthood. Subjects who rarely or never binged, approximately $90 \%$ of the sample, exhibited more mature views and behaviors regarding alcohol all along and encountered 
fewer consequences from consuming alcohol. How subjects in each pattern differed in their overall emotional maturity was not studied.

Researchers have also suggested that binge drinking may be one step of the process of maturing for some students. It has been argued that binge drinking needs to be studied apart from the use of other substances due to the more erratic individual patterns of alcohol use and to the possibility that the behavior may be developmentally appropriate for a subpopulation of students (Schulenberg et al., 2001). From this latter perspective, binge drinking is viewed as a means to explore risk taking and pursue other developmental milestones of young adulthood, but there exists within this path a strong potential for outcomes that are both desired or unwelcomed. Thus, some students may be encouraged by the positive personal experiences of binge drinking episodes and seek opportunities for continued use if the alcohol-related consequences are seen as minimal or "worth it."

A clear example of the importance binge drinking can have in a student's life is the seeking of spring break destinations for the purposes of "partying" heavily. Students who went to a spring break destination specifically for the binge drinking opportunities drank significantly more than students who went to same the destination for other reasons (Smeaton, Josiam, \& Dietrich, 1998). This finding also suggests that it is not just the culture or environment of drinking that leads to more dangerous levels of alcohol consumption. Individual expectancies 
of positive experiences arising from excessive alcohol use can play a key role as well. When young adults anticipate that alcohol will improve their lifestyle and socialization, they are more likely to abuse alcohol on a consistent basis (Mooney, Fromme, Kivlahan, \& Marlatt, 1987; Stacy, Wildaman, \& Marlatt, 1990).

Binge drinking can hold a completely different meaning for other college students who may find that the effects of alcohol provide the means to another end or facilitate some form of expression. Haemmerlie, Montgomery, and Cowell (1999) noted how the drinking behavior of college students was influenced by their personal value systems. Students who saw binge drinking as an expression of their personal choice or as an accepted practice of the social norm, regardless of consequences, were more likely to binge drink. Durkin, Wolfe, and Clark (1999) also found that respect for authority and acceptance of conventional beliefs were negatively correlated to binge drinking.

Surprisingly, the authors from the latter study revealed that despite these beliefs and negative views of authority, frequent binge drinking was associated with students who reported strong attachments to their parents, refuting the explanations of binge drinking from attachment and social bond theoretical perspectives (Durkin et al., 1999). However, the quality of the college student's parental relationship is only one part of the story of how parents may influence their children's alcohol use. Knight et al. (2002) reported that parental drinking was related to the presence of alcohol abuse or dependence in their children. If a 
parent was a problem drinker, the likelihood of the child being diagnosed with alcohol dependence increased significantly, but alcohol abuse did not increase. Regardless of parental attachment or drinking habits, children's drinking patterns on campus, at home from college, with family, and in general may be remarkably distinguished from their parents' drinking (Rabow \& Neuman, 1984; Yu, 2003). An overview of the above findings suggests that the influence of parental alcohol consumption on their children's drinking habits continues to remain complex and offers little direction for improving alcohol prevention programs.

The variety of persistent binge drinking patterns exhibited by college students have been attributed to a number of factors, including differences in maturity or developmental stage, personal value systems, positive expectancies, and parental attachment and drinking. These factors noted above offer evidence of the multiple psychological influences on a student's binge drinking which may reflect or impact the overall EI of the student. The relationship of gender, race and other cultural variables to binge drinking patterns and consequences has also been examined in greater depth but with mixed results.

Gender differences. The relationship between gender and binge drinking has been one of the most studied in the alcohol literature. Some patterns that have emerged remain consistent throughout the years, but many are showing signs of change as the college drinking culture evolves. One of the most common observations has been that men drink at a higher quantity and frequency than 
women and, in general, have more problems associated with alcohol (Berkowitz \& Perkins, 1987; Hilton, 1987; Humara, \& Sherman, 1999; Poikolainen, TuulioHenriksson, Aalto-Setala, Marttunen \& Lonnqvist, 2001; Quigley \& Marlatt; 1996). However, women's rates of drinking are becoming increasingly similar to the rates of men.

Berkowitz and Perkins (1987) reviewed the literature on gender differences from 1975 to 1986 and found that differences in alcohol consumption rates between genders were no longer significant when body weight was controlled as a variable. Women also increased their rate of drinking significantly more than men as they transitioned to college. Mercer and Khavari (1990) reported a 33.9\% increase in beer consumption among college women from 1977 to 1985 with a decrease in the abstention rate. Recently, women's colleges have experienced a $33 \%$ increase in excessive drinking in the past 8 years while binge drinking rates among college students overall have remained unchanged (Wechsler, Lee, Kuo, et. al, 2002). The same study noted that women in coed colleges continue to drink at much higher rate than those at women's colleges.

In general, it appears that binge drinking rates decline for both genders as college students mature (Weingardt et al., 1998). This decline in drinking, along with alcohol-related problems, has been observed across both gender and family history of alcoholism, but individual trends in drinking for females and males need to be strongly considered (O’Neill, Gilbert, \& Sher, 2001). Upon closer 
examination of the data on binge drinking patterns and consequences, gender differences emerge that demonstrate the need for further study in this area. Some results seem contradictory, while other research findings become highly qualified when gender is included as a variable.

Wechsler, Isaac, Grodstein, and Sellers (1994) found that many students who initiate binge drinking their freshman year maintain their drinking habits as sophomores, but when those who start after their freshman year are examined by gender, distinct patterns are seen. Approximately one-third of freshman males who had not previously engaged in binge drinking began to binge drink during their sophomore year on a relatively light level, though few females began binge drinking as a sophomore. A similar result was reported in a survey by McCabe (2002) in which men had a higher quantity and frequency of alcohol consumption after their freshman year; the reverse trend was true for women. Another study demonstrated a possible maturing effect for men when they are seniors. Berkowitz and Perkins (1987) observed that men drink more frequently as a senior compared to men in earlier years, but they drank a lesser amount, had fewer consequences, and were more aware of their use reflecting a drinking problem.

Some variables have been used to qualify the relationship between drinking and gender. Krugman (1995) emphasized the role of alcohol in reducing shame and feelings of vulnerability for men. College men's reported alcohol consumption has been associated with masculine gender-role conflict especially 
when the personal struggle involves issues of power, success and competition (Blazina \& Watkins, 1996). Capraro (2000) concluded that the study of alcohol use among college men should move beyond drinking behavior to an understanding of the function of alcohol in relation to a more comprehensive model of men, including their value and belief systems, sense of masculinity, and socialization with women.

Ingra and Moos (1979) noted that personal factors, such as disposition, play more of a role in alcohol consumption for men than for women. The drinking habits of women are more likely to be a function of the environment or situation in which they are drinking. The influence of environment combined with a tendency to misperceive campus drinking norms may put women at risk for excessive drinking. It has been suggested that the increasing drinking rates of women may be attributed to women being more likely to overestimate the openness of their peers' attitudes towards drinking (Berkowitz \& Perkins, 1987).

If women are influenced more by the context of their drinking, it would be expected that some settings on college campuses would present a greater challenge to women than to men, although this has not been found to be true. Men in fraternities are at much greater risk for excessive drinking than women who live in sororities (McCabe, 2002). Although, frequent binge drinking was present in approximately 4 of 5 members of sororities and fraternities (Wechsler et. al., 1998), a recent figure of $86 \%$ of fraternity members were found to be binge 
drinkers with 64\% reported as frequent binge drinkers (Caudill et al., 2006). Drinking rates have been decreasing for residents of the greek houses in general, but binge drinking rates have not been declining for their members overall (Wechsler, Lee, Kuo, et al., 2002). In addition, student athletes who are members of the greek system report the highest rates of binge drinking over any other group on campus, regardless of gender (Meilman, Leichliter, \& Presley, 1999); greater alcohol consumption was observed among sorority and fraternity members than student athletes. When data from athletes are examined by gender, both female and male athletes have significantly more alcohol consumption, episodes of binge drinking, and alcohol-related problems than non-athletes (Leichliter et al., 1998). Differences in gender are also not clear when the focus is on the effect of binge drinking on intimate interpersonal relationships. Nezlek, Pilkington, and Bilbro (1994) studied perceived intimacy among male and female college binge drinkers and found that men who reported the least intimacy and described less responsive partners were the most frequent binge drinkers (3 or more binges per week). In contrast, women indicated more responsive partners and greater romantic intimacy as their binge drinking levels increased. However, the highest perceived intimacy was seen for women when they shared the same high binge drinking rate as their partner; men with the highest perceived intimacy shared a low binge drinking rate with their partner. 
It may be important to note that high binge drinking women should be distinguished from women who abuse other drugs. While perceived intimacy in relationships remains for binge drinking women, drug abusing women tend to be more socially withdrawn and isolated with greater mood instability, pessimism, and irrational thought (Butterfield \& LeClair, 1988). This distinction offers support for the need to isolate the study of college binge drinking from that of other drug abuse.

Ethnic differences. The most recent statistics on differences in alcohol consumption rates across racial groups indicate that White men and women have higher binge drinking rates than their non-White peers (Quigley \& Marlatt, 1996; Wechsler, Lee, Kuo, et al., 2002). Specifically, White college students report the highest percentage of binge drinkers at $50.2 \%$, followed by Hispanic students at 34.4\%, Native American Indians at 33.6\%, Asian/Pacific Islanders at 26.3\%, and African-Americans at 21.7\% (Wechsler, Lee, Kuo, et al., 2002). These percentages represent an increasing rate of binging since 1993 for AsianAmericans and to a lesser degree, Whites and African-Americans; significant decreases were noted for Hispanic and Native American Indian students (Wechsler, Lee, Kuo, et al., 2002). The data support the earlier results of a survey by Bennett, Miller, and Woodall (1999) which showed that Hispanic students engage in higher levels of binge drinking than other students of color. The authors also pointed out that non-Hispanic, students of color had the highest rates of 
abstinence. In addition, Hispanic women appear to be more at risk for college binge drinking than Hispanic men (McCabe, 2002).

Caution should be used when examining data about race and alcohol consumption because racial interpretations can be made that may need to be qualified by environmental and other personal factors, such as religious environment (Koopmans, Slutske, van Baal, \& Boomsma, 1999; Luczak, Wall, Shea, Byun, \& Carr, 2001). Some observations of the relationship between race and drinking patterns have been consistent across settings and considered suggestive of a genetic component. A study conducted by Perez, Eliseo, Marin, and Marin (1994) identified a significantly lower alcohol consumption rate among Latinas and Latinos than their Caucasian peers in an urban general population, paralleling the racial drinking patterns found in college settings. However, the drinking rates of Caucasians and African-Americans have varied across studies of college campuses which implies that more than just racial factors are at work. Humara and Sherman (1999) found no difference in alcohol consumption between African-American and Caucasian students at one college campus, contradicting the national college survey statistics of Wechsler, Lee, Kuo, et al. (2002).

Other demographic factors. Relatively little alcohol research has been conducted on other demographic characteristics, such as sexual orientation, age, socioeconomic status, and religious affiliation. The studies that have examined these variables have revealed that binge drinking rates are higher for lesbian and 
gay adolescents and college students than their heterosexual peers (Caldwell et al., 1998; Debord et al., 1998) and that among all age groups, young adults (ages 18-29) are more likely to drink at a level of alcohol abuse and dependence (Quigley \& Marlatt, 1996).

As demographic variables are studied together in an effort to better understand alcohol-related behavior, the need for additional research of psychological differences becomes clearer. When race, gender and socioeconomic status were looked at collectively in a general urban population, Jones-Webb, Hsiao, and Hannan (1995) showed that more drinking consequences and total drinking problems were observed for less affluent African-American males than less affluent Caucasian males and more affluent African-American males. In contrast, affluent Caucasian males listed significantly more alcohol-related consequences and problems than less affluent Caucasian males. Caetano and Herd (1984) reported that income, employment status, and education were not associated with drinking habits for African-Americans, but religious affiliation (in particular, Fundamentalism) was associated with more abstainers and light drinkers. Furthermore, family socioeconomic status, drinking behavior, and ethnic background only become risk factors for predicting the development of alcohol and other behavior problems in children of alcoholics when combined with several other factors (Ellis, Zucker, \& Fitzgerald, 1997). The presence of alcoholism alone in the families of college students at-risk for alcohol abuse also 
does not adequately predict students' alcohol consumption, consequences, or dependency symptoms (Quigley \& Marlatt, 1996).

Although some patterns seem to emerge, the study of alcohol use and demographic data must be kept in perspective and take into consideration other individual differences that have an impact on alcohol use. For example, having a positive expectancy of drinking appears to predict college drinking significantly better than demographic variables (Brown, 1995). If the expectations that an individual has for the effects of alcohol supersede demographic predictors, differences in individual psychological factors could hold the key to why excessive drinking and its consequences can vary in such extreme ways across students and within an individual over time. Binge drinking consequences will be examined in the present study with a focus on making connections to psychological variables that seem to be related to certain patterns of consequences. Additional demographic differences will be explored in later sections for alcohol-related consequences and the use of alcohol for perceived psychological benefits.

\section{The Nature of Binge Drinking Consequences}

Current statistics on alcohol-related consequences for college students suggest that there have been no changes in the frequency of problems associated with alcohol since 1997, nor has there been any change in students being able to recognize their drinking as being a problem (Wechsler, Lee, Kuo et al., 2002). It 
should be noted that similar surveys conducted by Wechsler and his colleagues had reported an increase in alcohol-related consequences between 1993 and 1997. In the 1997 survey conducted by Wechsler et al. (1998), the increase in consequences was reflected in 13 alcohol-related problem areas for both men and women with most increases being statistically significant. One in five students indicated that they had encountered five or more consequences which is a $22 \%$ increase since the 1993 survey; binge drinkers were found to experience higher rates of consequences than non-binge drinkers.

The 1993 survey by Wechsler, Davenport, et al. (1994) specified that binge drinkers, regardless of frequency, were 25 times more likely to endorse 5 or more alcohol-related consequences during the academic year than nonbinge drinkers. Only $3 \%$ of nonbinge drinkers endorsed having this level of alcoholrelated problems as compared to $47 \%$ of frequent binge drinkers. It should be noted that some students do encounter serious problems from nonbinge alcohol use which implies that consequences attributed to alcohol may be more complex than simply being an outcome of frequent excessive drinking (Caldwell, 2002).

Regardless of the large number reporting consequences, almost no students $(.2 \%)$ identified themselves as being a problem drinker (Wechsler, Davenport, et al., 1994), a finding that continues today (Wechsler, Lee, Kuo et al., 2002). This tendency to report consequences while denying "drinking problems" in addition to expressing little desire to change drinking habits (Caldwell, 2002) 
raises several questions. Further research is needed to explore how students can be aware of the consequences they attribute to alcohol yet find them acceptable to maintain in their life along with high levels of alcohol consumption. Schulenberg et al. (2001) revealed that negative consequences from alcohol use do not lead to a subsequent reduction in drinking for college students, but positive experiences with alcohol do predict increased drinking for the following week. Positive consequences were reported more than negative, regardless of the level of alcohol use, which may have implications for what fuels the magnitude of the college binge drinking problem. Students who binge drink apparently seek and experience desired effects from the alcohol which supersede whatever negative consequences they may perceive.

Despite the extensive work on alcohol-related consequences by Wechsler and his colleagues, categorical differences or patterns of consequences have not been highlighted in their results. Other researchers have begun to take a closer look. O'Neill et al. (2001) recommended that multiple paths be examined when studying alcohol-related consequences among young adults who engage in heavy drinking. Vik et al. (2000) conducted a principal components analysis of alcoholrelated problems which yielded three factors (careless behavior, risky/reckless behavior, and authority problems). These three factors seemed to follow a progression of severity and were associated with psychological variables, frequency of use, alcohol expectancies, and age of initiating drinking. 
Miller, Tonigan, and Longabaugh (1995) take a different approach to studying consequences. They developed an instrument (The Drinker Inventory of Consequences; DrInC) to specifically categorize alcohol-related problems within a framework that could facilitate alcohol prevention and treatment efforts. The authors also supported the notion that alcohol-related consequences could be reduced without a corresponding drastic change in consumption, based on a finding by Chick, Ritson, Connaughton, Stewart, and Chick (1988). The categories of alcohol-related consequences, proposed by Miller et al., will be used in the present study to examine how they may be related to EI factors. The following is a description of each category along with recent data regarding specific consequences within the category.

Physical. Miller et al. (1995) described physical consequences as those adverse physical states, both acute and chronic, that arise from alcohol abuse; injuries occurring while intoxicated would also fall in this category. Binge drinkers have been shown to be prone to brain damage (Hunt, 1993), antibody suppression (Han \& Pruett, 1995), memory loss (Browning et al., 1992), stroke (Altura et al., 1998), and injury (Shepard \& Brickley, 1996). Those who binge drink at a higher frequency are also at an increased risk of personal physical harm (Wechsler, Davenport et al., 1994). Over 150,000 college students state that they have had an alcohol-related health problem in the past year, and approximately 1,400 college students die and 500,000 are injured each year from unintentional, 
alcohol-related accidents (Hingson, Heeren, Zakocs, Kopstein, \& Wechsler, 2002).

What may be a great concern to prevention researchers is the steady increase of physical consequences in the past decades. Hanson and Engs (1992) surveyed college students nationally over a period of 9 years and found growing rates of hangovers and vomiting due to alcohol. Wechsler, Lee, Kuo et al. (2002) reported in their national surveys conducted between 1993 and 2001 that there was a significant increase in the number of college students becoming hurt or injured after binge drinking.

Social responsibility. Alcohol-related consequences related to social responsibility refers to problems meeting the demands and responsibilities of the various roles an individual has, such as student or employee (Miller et al., 1995). Problems can range from tardiness and absenteeism to disciplinary action, financial difficulties, and not fulfilling obligations or expectations. In their national survey, Hanson and Engs (1992) noted increases over a course of 9 years of students reporting missing classes to drink alcohol, lower grades, and disciplinary action from college administration due to alcohol. Although there was a decrease in students coming to class intoxicated, a strong increase was observed in those missing class because of a hangover. Wechsler, Lee, Kuo, et al. (2002) also found significant increases in academic problems that were attributed to alcohol use between 1993 and 2001. 
Intrapersonal. Intrapersonal consequences are defined by Miller et al. (1995) as those inner, subjective alcohol-related problems that are identified by an individual through her or his own self-perceptions. Others may not be aware of the individual's internal experience of psychological distress which could include negative affect, mood states, or personality changes. Problems with moral, spiritual and personal growth, as well as an individual's ability to engage in leisure activities, are also characteristic of intrapersonal consequences. Bonin, McCreary, and Sadava (2000) surveyed a community based sample of young adults and observed significant associations between problem drinking behaviors (i.e., binge drinking) and self-reports of depression and loneliness.

The results of the Bonin et al. survey support earlier research findings regarding connections between alcohol use and depression and loneliness (Ackerlind, \& Hornquist, 1992; Brennan, Moos, \& Mertens, 1994; Turnbull \& Gomberg, 1988), but other intrapersonal variables have been lacking in the binge drinking literature. Research in this area is particularly needed because more "internal" or intrapersonal problems from alcohol use are reported than "external" (Plant, Miller, Thornton, Plant, \& Bloomfield, 2000), depending on an individual's racial identity (Humara, \& Sherman, 1999). Additionally, suicide attempts in as many as $1.5 \%$ of college students in the past year have been attributed to alcohol and other drug use (Presley, Leichliter, \& Meilman, 1998). 
Impulse control. One of the most studied categories of alcohol-related consequences, developed Miller et al. (1995), is impulse control. A wide range of consequences that appear to stem from impulsiveness when overdrinking are included in this category, such as physical altercations, an increase in other substance use, unhealthy risk taking, drunk driving and associated accidents, harm to others and/or property, and legal problems or arrests.

Perhaps the most serious of these consequences has been the numerous alcohol-related traffic fatalities and injuries. In an article posted by CNN interactive ("Data points," 1997), a surprising statistic captured the scope of the drunk driving problem. The article reported that there is an average of one death every 30 minutes due to an individual driving under the influence of alcohol. Although there was a substantial decrease in the rate of drunken driving deaths during the 1980's and no significant change in the 1990's, Mothers Against Drunk Driving announced that the number of deaths attributed to drunk driving has actually increased from 2001 to 2002 (“MADD,” 2002).

Drunk driving among college students has been a noteworthy consequence of alcohol use (Borges \& Hanson, 1993); an estimated one third of college students drink and drive (Wechsler et al, 1998). In 1994, the National Highway Traffic Safety Administration reported that drunk driving among young adults was a disturbing, high-risk behavior needing attention. Their report communicated a strong concern despite a significant decrease over a 9-year 
period in the number of drunken-driving-related consequences among college students (Hanson \& Engs, 1992). The concern is strengthened by statistics that show approximately $30 \%$ of drunk driving deaths involved young adult drivers (ages 16-24), but only 15\% of all licensed drivers fall within that age range (Quigley \& Marlatt, 1996). Binge drinking has been connected to the problem due to college students being five times more likely to drive while intoxicated if they engage in binge drinking on either a frequent or infrequent basis (Wechsler, Davenport, et al, 1994). Not much is known as to what leads young adults to impulsively risk their lives with drunk driving. However, Beck and Treiman (1996) studied adolescent drinkers and their alcohol-related consequences and found an association between defiance of school rules and drunk driving; the need for peer approval had only a moderate influence on those who drove while intoxicated.

Another serious and pervasive alcohol-related consequence that reflects impulsivity is high-risk sexual behavior, including sexual assault. Meilman (1993) showed that alcohol intoxication played a role in sexual activity for $35 \%$ of college students during their college years; $18 \%$ and $15 \%$ of students blamed alcohol use for their having sexual intercourse and unprotected sex, respectively. The abandonment of safe-sex practices was also related to alcohol use in homosexual men (Paul, Stall, \& Bloomfield, 1991). 
A survey conducted by The Henry J. Kaiser Family Foundation (2002, February) indicated that alcohol and other drugs were involved in the sexual activity of $37 \%$ of young adults (ages 18-24), consistent with the findings of Meilman. Other results from the survey determined that $31 \%$ of young adults under the influence of alcohol or other substances went beyond their sexual limits. Approximately a quarter of the subjects had unprotected sex or was concerned about possible physical consequences and pregnancy. Furthermore, the belief that choices about sexual activity could be decided while using alcohol or other drugs was deemed acceptable by $21 \%$ of participants in the survey. This statistic suggests that some substance-related impulsive behavior is anticipated and considered appropriate by young adults, regardless of the number of their peers who experience harm from alcohol-related sexual activity.

As previously noted, the vast majority (86\%) of administrators who were surveyed from colleges with heavy drinking believed that sexual assault was a major problem on their campus (Wechsler et al., 1995). The National Center of Addiction and Substance Abuse (1999) estimates that at least one party is under the influence of alcohol in 46 to $75 \%$ of sexual assault and rape cases on college campuses. The problems associated with sexual behavior and alcohol use are clearly not fading with time and prevention efforts. Unfortunately, the alcoholrelated impulsivity that is present in many of these acts of sexual violence can often be extended to violence that arises in other areas of campus as well. 
Wechsler et al. (1995) reported that a majority of college administrators from institutions with heavy student drinking describe significant problems with physical assaults and damage to property on campus. Binge drinking has been shown to be related to problems with campus police, property damage and overall violent behavior (Engs, Diebold, \& Hanson, 1994; Valois, McKeown, Garrison, \& Vincent, 1995; Wechsler, Davenport et al., 1994) and these problems appear to have been steadily increasing throughout the years. In their national survey, Hanson and Engs (1992) saw increases over a 9-year period in damaged property, pulling false fire alarms, having trouble with the law, and physical altercations following drinking. The trend continued for college binge drinkers between 1993 and 2001 with significant increases in getting into trouble with the law (Wechsler, Lee, Kuo et al., 2002).

When binge drinkers are under the influence of alcohol, approximately $10 \%$ have caused property damage and between $4-6 \%$ got into trouble with the law, but these figures are much higher for frequent bingers (Wechsler et al., 1998). Again, as the frequency of binge drinking rises, the potential for serious consequences also appears to increase. Impulsiveness and thrill-seeking has been present in college students who engage in occasional and frequent binge drinking, and frequent bingers are more disinhibited and prone to drinking in high risk situations than all other drinkers (Ichiyama \& Kruse, 1998). 
Interpersonal. Miller et al. (1995) designated the last of the categories of alcohol-related consequences as interpersonal, focusing on problems that occur within a drinker's relationships as a result of alcohol. These consequences can range from embarrassing someone or engaging in interpersonal conflict to prolonged deficits in the quality of romantic relations. Criticism for a romantic partner's alcohol use increased during the late 1980's (Hanson \& Engs, 1992) which may reflect the difficulty frequent binge drinkers tend to have managing intimacy in their relationships (Nezlek, Pilkington, \& Bilbro, 1994). Wechsler, Lee, Kuo et al. (2002) reported that overall interpersonal problems due to alcohol use have been increasing since 1993. Furthermore, a high rate of non-binge drinking or abstaining peers indicated that they have personally experienced the negative consequences of someone's alcohol use.

Students who are non-binge drinkers and live on campuses with high proportions of binge drinkers are particularly susceptible to the secondhand effects of alcohol intoxication (Wechsler, Davenport, et al., 1994). Wechsler et al. (1998) showed that approximately $75 \%$ of college students have encountered alcohol-related annoyances and problems attributed to their peers' drinking. Most students have had their sleep disturbed or needed to care for a peer who was highly intoxicated, but $28.6 \%$ of students were directly confronted with verbal insults. The authors stated that $19-24 \%$ of students are under the influence of alcohol when they are engaging in some form of verbal aggression. On a more 
serious level, significant verbal aggression has been experienced by $22 \%$ of college students, physical altercations by $12 \%$, and unwanted sexual advances by $20 \%$ (Wechsler et al., 1995). Hingson et al. (2002) found that over 600,000 college students are victims of assault by an intoxicated student each year, and over 70,000 are sexually assaulted or date raped. Although the number of students affected by the secondhand effects of binge drinking has remained relatively stable between 1993 and 1998, Wechsler et al. (1998) noted significant increases in those reporting a serious interpersonal conflict, unwanted sexual advances, and the need to care for an intoxicated peer. Supportive relationships are critical to success in college, but relationships become filled with high levels of interpersonal stress and strain under these conditions of binge drinking.

Consequences over the college years. When examining the alcohol-related consequences of college binge drinkers, consideration should be given to the students' age and developmental level as other important factors linked to the presence of problems. Alcohol-related problems experienced by first-year college students have been predicted by reports of stress and changes in drinking habits (O’Hare \& Sherrer, 2006). According to the 2002 college binge drinking survey conducted by Wechsler, Lee, Nelson et al., students who drink alcohol under the legal drinking age are at great risk. When compared to legal-aged college drinkers, underage drinkers have more physical injuries and blackouts due to alcohol, and they engage in more property damage and a wide range of behaviors 
that are regretted. Underage drinkers also were significantly more involved in police action than legal-aged drinkers, but it was not noted how much police trouble was due to violations of underage drinking laws.

Differences between underage and legal-aged drinkers may be less than reported. The above survey doesn't account for students being permanently separated from school for alcohol-related problems prior to legal drinking age, thereby reducing the number of "legal" students who have had problems due to drinking. In contrast, underage drinkers have fewer problems than legal drinkers with drunk driving, missing classes, and having unprotected sex. It should be noted that since 1993, underage drinkers had increases in all of the alcohol-related consequences measured by the researchers, although having unprotected sex and falling behind academically were not statistically significant.

O'Neill et al. (2001) supported the finding that students early in their college career, typically underage drinkers, tend to have more problems attributed to alcohol than students in upper classes. The link between heavy alcohol use and consequences was also observed to weaken as students age, suggesting that the volume of alcohol consumption should not be considered the only variable involved in consequences. The trend continues beyond the college years as alcohol-related consequences tend to exhibit a significant decline following college. Schulenberg et al. (2001) showed that future psychosocial problems 
could not be predicted by drinking as a young adult. The authors believed this observation represents a developmental phenomenon for binge drinking.

The above results are consistent with the maturing effect, described by Fillmore (1988), which proposes that decreased alcohol-related consequences are associated with an individual's development and maturation. There are exceptions to this theory as demonstrated by heavier college drinkers being prone to ongoing heavy drinking and associated problems following their college years (O’Neill et al., 2001; Windle, 1988). As a result, O’Neill et al. (2001) suggested that the study of problems resulting from heavy alcohol use should not be one dimensional and allow for other possible trends based on individual differences.

Demographic differences. In addition to age and class year, other demographic factors appear to be related to the trends observed in alcohol-related consequences. The variety of patterns that arise from the interaction of gender and alcohol consumption are continued in the complex associations between gender and binge drinking consequences. For both men and women, problems due to excessive alcohol use grew significantly between 1993 and 1998 (Wechsler et al., 1998). Although as previously noted, men typically anticipate and report more alcohol-related consequences than women (Berkowitz \& Perkins, 1987; Liu \& Kaplan, 1996), a difference that continues regardless of the level of alcohol consumption (Plant et al., 2000). 
Men tend to report more interpersonal problems including physical altercations and being criticized for drinking, but both women and men have been shown to have the same rate of academic problems resulting from alcohol use, (Berkowitz \& Perkins, 1987). Liu and Kaplan (1996) added that women experience more physical consequences from their initial alcohol use while physical injuries are more likely to increase for men with continued drinking. As women progress through their college career, the impact of alcohol on their academic performance lessens, but interpersonal consequences increase (Berkowitz \& Perkins, 1987). Regardless of the type of consequence, men and women who engage in heavy binge drinking to a problem level will most likely continue excessive drinking over time (McCabe, 2002).

Berkowitz and Perkins hypothesized that the fewer consequences reported for female problem drinkers may be due to sensitivity to environmental pressures and criticism which inhibits problem behavior. However, the belief that men actually experience more consequences than women has been met with some debate. Some researchers have concluded that gender is not a predictor of alcoholrelated problems when other variables are included in the model, such as drinking motives (Carey \& Correia, 1997). Giancola (2002) surveyed the literature on alcohol, aggression, and gender and found inconsistent results when researchers attempted to identify different levels of aggression between men and women when alcohol is consumed. Recently, Wells, Speechley, Koval, and Graham 
(2007) demonstrated that there was a stronger relationship between fighting after drinking and excessive alcohol consumption among female adolescents and young adults than males. Thus, the relationship between gender and alcoholrelated consequences remains multifaceted.

Thom (1986) offered another perspective by stating that women may encounter more alcohol-related consequences than reported in the literature because they avoid awareness of the problems that stem from their excessive alcohol use, a possible by-product of societal pressures. Society may have a biased view that female abstainers are more acceptable than male abstainers, but for alcohol problems, female problem drinkers would be considered more deviant as compared to men with equivalent problems (Moos, Moos, \& Kulik, 1977). Plant et al. (2000) conducted a European collaborative study on alcohol problems in which they concluded that women are more apt to describe "internal" or intrapersonal problems and are less likely than men to exhibit "external problems" (i.e., interpersonal conflicts) and attribute problems to alcohol. The gender difference appeared most pronounced for external problems. Male gender was found to be a predictive factor for acute alcohol problems along with binge drinking, quantity of alcohol consumed, and being young. The authors support focusing future research away from the impact of quantity and frequency of alcohol consumption towards gathering more data on how individuals understand their drinking and associated problems. 
Bonin et al. (2000) demonstrated the importance of focusing on the emotional and interpersonal consequences and factors related to excessive alcohol use in addition to gender. They found that depression and loneliness were significantly related to problem drinking (i.e., binge drinking) in young adults from a community-based sample. Gender differences were not significant when quantity and frequency of alcohol consumption were controlled. An exception was identified as the frequency of intoxication was considered. Women who selfreported to be lonely became intoxicated more frequently than lonely men. There were no suggestions as to why this finding might have occurred, but the need to examine alcohol as a psychological coping tool is evident.

Racial identity has rarely been studied in the context of type of alcoholrelated consequence. One study by Humara and Sherman (1999) found a significant interaction between race and type of alcohol problem reported. Caucasian, binge-drinking college students described more interpersonal problems due to alcohol use than intrapersonal, but African-American binge drinking students reported more intrapersonal problems than interpersonal. The authors suggested that this result could be due to African-American students leaning toward behaviors that tend to be seen as "non-White." Race was not an issue for alcohol-related problems being attributed to interpersonal sources significantly more than intrapersonal. As racial and gender patterns of alcoholrelated consequences seem to provide more questions than answers, a greater 
depth of understanding of emotional and interpersonal correlates appears necessary.

Other factors involved in alcohol-related consequences. The numerous types and trends of alcohol-related consequences, as well as associated variables, suggest that it may be too simplistic to say that these consequences are due exclusively to excessive alcohol use (Caldwell, 2002). In actuality, the subjective experiences of the college drinker (i.e., perceptions of being "high") have been found to play a more important role in problems attributed to alcohol than binge drinking alone (O'Neill et al., 2001). A binge drinker may be reacting to a number of psychological and social factors unique to the individual that become expressed through consequences of alcohol use. Nagoshi (1999) studied college students' using the Impaired Control Scale (ICS) which showed that perceived control over alcohol use could predict alcohol-related consequences but not alcohol consumption. Several personality characteristics were correlated with the ICS, especially impulsivity and proneness to depression. Another example of a personal variable that has an impact on the outcome of a drinking episode is how drinkers see themselves in relation to their peers. Beck and Treiman (1996) demonstrated that adolescents' perceptions of the drinking norms among their closest friends were strongly correlated with alcohol consumption and consequences. 
The lack of alcohol-related consequences in some binge drinkers also seems to strengthen the argument against the intoxicating effects of alcohol being the sole cause of problems. Turrisi et al. (2000) explored why not all college students who binge drink experience alcohol-related consequences. The authors considered individual or situational differences as having possible effects on the presence of consequences beyond that of excessive alcohol use. Incoming freshmen were studied over a period of 30-50 days and were assessed for their cognitive beliefs and expectancies about alcohol and the subsequent alcoholrelated consequences that arose. Negative consequences were present more in students who maintained positive expectancies about alcohol and its ability to facilitate socialization than those students who held less positive expectancies. In addition, it appeared that students who described greater communication with parents about alcohol were less likely to have positive expectancies.

Consequences were not differentiated in the study. It is also not known if the positive expectancies about alcohol reflected an emotional need (i.e., the hope to improve socialization to reduce loneliness, hopelessness or anxiety), although the importance of how each student makes meaning of drinking is highlighted.

The findings of Turrisi et al. support earlier research by Carey and Correia (1997) who revealed that drinking motivations are better predictors of alcohol problems in college students than heavy drinking and that the desire to improve socialization is highly associated with consequences. In terms of affective 
variables, consequences and heavy drinking were exhibited more in students who described drinking to remove an undesirable mood state (negative reinforcement) than those who endorsed motives to drink to attain a desired state (positive reinforcement). Cox and Klinger (1990) proposed a model of heavy drinking consistent with the notion that both positive and negative reinforcement are the motivating forces for alcohol problems on both internal (i.e., emotional) and external (i.e., socialization) levels. The model doesn't address if individual differences could be broken down into categories associated with the onset of specific problem types, but it does encourage further examination of the use of alcohol for interpersonal management and emotional coping.

Research has shown that men who encounter alcohol problems are more likely to use alcohol to improve socialization and to manage negative affect than men without problems (Glynn, LoCastro, Hermos, \& Bosse, 1983). Cronin (1997) also observed that the social environment is an important factor in alcohol consumption, but alcohol-related consequences were best predicted by students who engaged in drinking for mood enhancement. The relationship between alcohol problems and the use of alcohol for affect and mood management may be most visible among people who are unemployed. The stress and negative affect that arises from unemployment may be the key factor for this segment of the general population having had the highest binge drinking rate and most alcoholrelated consequences (Crawford, Plant, Kreitman, \& Latcham, 1987). For college 
women, alcohol-related consequences could be predicted by the sole use of avoidant coping mechanisms to relieve stress at school and work (Simpson \& Arroyo, 1998). Using alcohol to manage affect, mood, and/or stress seems to be a first step towards the development of consequences, but there may be other factors involved. Baer (2002) reviewed the literature on alcohol-related problems and individual differences. Some inconsistent support was found for a connection between alcohol-related problems and drinking to reduce negative affect.

Vik et al. (2000) added that personality factors were related to differences in the seriousness of alcohol-related consequences. Students who reported having more serious consequences (the authority problem group) exhibited less neuroticism (i.e., negative emotionality, unstable affect, problems coping) and higher extraversion than the other two problem groups (the careless and risky behavior groups). The authority problem group also had a higher alcohol consumption rate than all other groups. This group and men both scored higher on measures of positive interpersonal expectancies from alcohol use than the other two groups and women. The group with the least serious consequences (careless) reported the least usage of alcohol for reducing social distress. The study illuminates the need to consider how individual psychological differences (i.e., differences in management of affect and interpersonal relations) can lead some college students to be more vulnerable to specific types of alcohol-related consequences than those with similar drinking patterns. 
Evidence has been cited above to support emotional and interpersonal variables as potentially playing key roles in the onset of binge drinking consequences and, to some extent, in the type of consequence. The persistence of the consequences and denial of having alcohol problems suggests that alcohol may be psychologically and interpersonally important to some college students. The combination of the stress of college with the maturity level of older adolescence may put significant strain on students' psychological ability to cope. If binge drinking is viewed as a means to help express or meet a psychological need, understanding differences in students' abilities to function on emotional and interpersonal levels would be an initial step toward improving their overall mental health and reducing alcohol consequences. Extensive research has been conducted on the role of alcohol and binge drinking in the emotional and interpersonal life of individuals.

\section{Binge Drinking as a Psychological and Interpersonal Tool}

Alcohol in the culture of the United States has been long considered an integral part of social gatherings as well as a means to "relax." In fact, a moderate use of alcohol is correlated with positive psychosocial functioning (Labouvie, 1990; Silbereisen \& Noack, 1988). It would follow that alcohol could be seen by some individuals as a positive tool for achieving some psychological and interpersonal goals. Unfortunately, these motivations for drinking, when taken to 
an extreme, may lead to serious consequences and reflect a more negative or vulnerable aspect of the individual.

Young adults are more likely to abuse alcohol and sustain heavier drinking patterns when they hold positive expectancies about alcohol's ability to manage mood states and improve socialization (Mooney et al., 1987). Adolescents who report emotional problems also tend to use substances at rates significantly higher than there peers (Tschann et al., 1994). In addition to being male and drinking to become intoxicated, Schulenberg, Wadsworth, O’Malley, Bachman, and Johnson (1996) reported that low self-efficacy was connected to increased binge drinking rates as adolescents moved into young adulthood. The authors proposed that binge drinking may serve as a more immediate, external antidote for the feelings of helplessness and powerlessness that come with low self-efficacy. Alcohol-related problems associated to low self-efficacy were not examined, but young adults with a high socioeconomic status have been found to describe a heightened sense of power and self-importance from alcohol use (Liu \& Kaplan, 1996).

The stress and anxiety inherent in the transition from adolescence to young adulthood may be exacerbated by the additional stressors that can occur from starting college. The psychological impact of these transitions could have implications for the high drinking rates of first-year students. Sax, Gilmartin, Keup, Bryant, and Plecha (2002) surveyed college students at the beginning and end of their first academic year. The percentage of students feeling that their 
emotional health was above average declined from $52.4 \%$ to $44.9 \%$ upon completion of the first year; self-assessments of physical health showed a similar decline. Increases were noted in the number of students feeling overwhelmed and depressed in the past year compared to the year prior to the start of college.

The first year of college takes an apparent psychological and physical toll on students which may set them up for seeking drinking as the means to cope with or express their distress. Berkowitz and Perkins (1987) showed that both men and women exhibit significant increases in their alcohol consumption during the transition to college. This finding led the authors to hypothesize that psychological factors could more likely be the catalysts to increased drinking than environmental factors. Depression, stress, and irrational beliefs have been related to alcohol problems in college students (Camatta \& Nagoshi, 1995). Transitions are thought to be so difficult and stressful that Maggs (1997) proposed that some students use substances to an extreme in order to prolong their college years and delay the painful transition of taking on adult roles.

Several theoretical considerations have been proposed regarding the psychological reasons for excessive alcohol and drug use. The focus of the theories typically revolves around the need to consume substances in order to selfmedicate or manage problems with anxiety, anger, or depression (Goleman, 1995). Perhaps one of the most well-known theories espousing a similar idea is social learning theory. Alcohol use is viewed in social learning theory as a learned 
method of coping when other coping mechanisms are inadequate or unavailable (Abrams \& Niaura, 1987; Bandura, 1969). Smith, Abbey, and Scott (1993) provided support for the relationship between alcohol and coping when they studied reasons for drinking alcohol. Affect management and coping were cited as common motivations for drinking, but several other reasons were listed as well, including to improve socialization, increase interpersonal confidence, and experience positive affect or enjoyment. Further detail of some of the dominant interpersonal and emotional reasons for drinking will reinforce how the use of alcohol among college students may reflect psychological needs and potential problems.

Facilitation of interpersonal relations. Many researchers have emphasized the critical role that alcohol seems to have in the social environment and interpersonal relationships of the college drinker. Baer (2002) concluded that the onset of drinking for college students at a level surpassing their noncollege peers can be partially attributed to social factors and a tendency towards extraversion in students. The interpersonal role of alcohol has been portrayed as both positive and negative, but despite the often severe costs, many students appear to place a strong value on alcohol when socializing on campus. Binge drinking has been found to be associated with those who see alcohol use as a social convention and a reflection of their personal values (Haemmerlie et al., 1999). 
The meaning and importance of alcohol may lie in how socialization is connected to the culture of drinking on college campuses. Drinking has been viewed as beneficial for some students because of the interpersonal relationships being fostered through alcohol-related, social gatherings (Fondacaro \& Heller, 1983). In fact, students who have lowered their drinking rate from an excessive level have been reported to be more withdrawn from the campus social life than when they were drinking heavily (Moos et al., 1977). Fondacaro and Heller (1983) support the perspective that there will be different modes of psychological adjustment for students transitioning into the college social environment and that using drinking to encourage socialization may be positive for some.

Maggs (1997) acknowledged that drinking alcohol may facilitate socialization during the critical transition to college, but the consequences of excessive use present physical and mental health costs to students that outweigh the benefits. Among adolescent drinkers, those who have a higher frequency and quantity of alcohol consumption are more likely to use alcohol to develop social relationships; parental influences appeared to have no significant impact in the type of drinking (Beck \& Treiman, 1996). The research on college students has also consistently shown that using alcohol for socialization is related to high levels of drinking. Binge drinkers use alcohol to facilitate socialization significantly more than nonbinge drinkers, and frequent binge drinkers demonstrated a higher use of alcohol for socialization than occasional binge 
drinkers (Ichiyama \& Kruse, 1998). Cronin (1997) stated that drinking in the context of a social gathering was the predominant cause of excessive alcohol consumption among the college students assessed.

The social factors that encourage interpersonal relations in drinking situations may also be considered sources of pressure if students drink in order to connect to others. Collins, Parks, and Marlatt (1985) suggested that excessive drinking may be promoted when young adults seek to follow the model of their peers who drink at a heavier rate than their own. Approximately $70 \%$ of AfricanAmerican adolescents have drinking habits that mimic their best friend's alcohol use (Forney, Forney, \& Ripley, 1991), and young adult males typically share the same drinking patterns as their closest friends (Liu \& Kaplan, 1996). Ichiyama and Kruse (1998) found that binge drinkers are more likely to report social and developmental pressures (including peer acceptance) to drink than nonbinge drinkers. The authors used the results to support the Social Context of Drinking construct (Beck, Thombs, \& Summons, 1993) which espouses the theory that drinking is a product of motivational and situational social factors.

Drinking may be seen as means to initiating relationships and maintaining connections to peers and close friends, but other studies have added that alcohol could be a factor in the progression or deepening of relationships. In a survey sponsored by the Henry J. Kaiser Family Foundation (2002), the above-mentioned findings on the influence of binge drinking peers on the drinking rates of young 
adults were corroborated, although alcohol and drugs were reported to also be used to reduce anxiety in sexual relations by $16 \%$ of the young adults. As previously noted, Nezlek et al. (1994) examined binge drinking, sociability, and intimacy among college men and women due to inconsistencies in the research literature. Self-reports of romantic intimacy in relationships were related to binge drinking levels depending on the gender of the student. Higher binge drinking levels in women reflected greater intimacy and perceived partner responsiveness while the reverse was true for men. The lack of binge drinking was related to less intimacy and disclosure in relationships leading the authors to propose that moderate binge drinking could be considered normative. Supporting this view of binging was the observation that relatively little socialization took place during the binge episodes for those students binging at a moderate frequency, suggesting that the majority of these students' social lives did not occur in a drinking context. Furthermore, the caliber of the social lives of students was not related to the quantity of alcohol. Nezlek et al. argue that the act of drinking to fit into the perceived norm of the binge drinking culture on campus could be evident in healthy college students.

Drinking to facilitate socialization and interpersonal relationships is related to higher levels of alcohol consumption, but the idea that binge drinking alone implies social or other problems has been refuted. Fondacaro and Heller (1983) found that overall mental health symptoms in college students are not as 
linked to alcohol use when considering other social factors unique to the individual drinker (i.e., social withdrawal). Students who drink for interpersonal purposes appear to be socially competent, and drinking in social situations could indicate that a student is engaging in a positive social life. Further research is needed that goes beyond the intoxicating effects of alcohol to determine what personal factors, interpersonal deficits, or psychological needs may be associated with the relational consequences that some experience when binge drinking. Affect management. Alcohol has the distinct characteristic of being promoted throughout history as perhaps the most popular agent for soothing uncomfortable feelings, tolerating pain, and fostering a "party" spirit. Observations such as these have led some theorists to believe that alcohol consumption is primarily motivated by the desire to manage positive and negative affect as well as overall mood states (Cooper, Frone, Russell, \& Mudar, 1995; Lang et al., 1999). Carpenter and Hasin (1998) offered empirical support for this theory in a community sample. The frequency of drinking to intoxication was positively correlated with the intention to use alcohol to cope with negative affect, and the subjects' overall drinking rate was positively correlated with the motive to drink for enjoyment. The most frequently cited reason for drinking, regardless of the level of alcohol problem (ranging from no problem to severe), was to create a feeling of enjoyment; those with no diagnosed alcohol problem had the strongest association to this drinking motive. 
Factors that suggest poor affect management, such as impulsiveness, sensation seeking, and low self-control, have also been connected to binge drinking in college students and young adults (Beck, Thombs, Mahoney, \& Fingar, 1995; Quigley \& Marlatt, 1996). Additionally, the rate of alcohol consumption and problems in college students appears to increase with the presence of depression, anxiety, or tension (Camatta \& Nagoshi, 1995; Carey \& Correia, 1997). Students who encounter emotional, mood, or behavioral difficulties seem to look to alcohol to help them cope with their symptoms. It is possible that there may be some quality about alcohol (i.e., its intoxicating or disinhibiting properties) that may be appealing to an individual who has few personal psychological resources or tools. It is not known to what extent alcoholrelated consequences may be generated by specific deficits in affect management more than the alcohol abuse alone.

There is some evidence that the interaction between the chemical properties of alcohol and situational variables has an influence how a drinking episode is emotionally processed. Melia, Corodimas, Ryabinin, Wilson, and LeDoux (1994) support the proposal that negative affect may decrease because of alcohol's ability to interfere with higher-order cognitive abilities. As a result, alcohol can suppress the effect of emotional cues, such as those stimulating fear. However, Lang et al. (1999) stress that an essential component of alcohol's potential to reduce negative affect is the presence of a distraction that is perceived 
to be non-negative while the alcohol is impairing cognitive processing. A negative distracter or the lack of any distracter could exacerbate the negative affect. With higher-order processing impaired, the distracter may need to be obvious and uncomplicated for the negative affect to be impacted. There is no definitive neuropsychological process that explains how alcohol encourages positive affect (Greeley \& Oei, 1999). Alcohol's effect on emotion and mood may therefore be multidimensional and subject to the perceptions of the individual and context of the drinking situation. This understanding of the interaction between alcohol and the individual implies that how an individual perceives and processes emotional cues (both external and internal cues) will be dependent on their alcohol intake and existing emotional functioning or EI. Thus, deficits in EI may lead a binge drinker to be vulnerable to negative affect and the subsequent alcohol-related consequences.

Tension reduction. Media advertising and popular opinion have promoted the idea that alcohol can be used to provide a sense of relaxation or relief from stress, although much of the research in this area has been qualified. Cappell and Greeley (1987) derived a hypothesis on the use of alcohol for tension reduction from prior studies and theory on drive reduction. They believed that a process, similar to negative reinforcement, was involved in alcohol consumption in which stress, tension, or other aversive experiences could be alleviated by drinking. An easing of tension by alcohol was thought to encourage continued use. 
Individuals who experience significant psychological distress and use alcohol as their primary stress management tool were shown to be particularly vulnerable to alcohol abuse and dependency (Kessler et al., 1997). Among firstyear college students, reports of stress have predicted alcohol problems (O'Hare \& Sherrer, 2006), and frequent binge drinkers have been distinguished from all other drinkers by their motivation to use alcohol as a coping tool for stress and uncomfortable affect (Ichiyama \& Kruse, 1998). Excessive alcohol use has been associated with relief drinking and relief smoking in young adults, but many other variables (e.g., social support, GPA, self-esteem, number of stressors, and ego defense style) did not predict drinking rates (Poikolainen et al., 2001).

Drinking for the purposes of relief or tension reduction appears to stand out as an indicator of heavy alcohol use and may need to be considered separately from other drinking motives. Brown (1985) described how college students who drank alcohol expecting tension reduction were more likely to be problem drinkers than students who drank to improve socialization. It may be important to further consider if different drinking expectancies (i.e., tension reduction vs. socialization) can lead to different drinking rates, they may also predict different psychological and interpersonal alcohol-related consequences.

Several other research studies have demonstrated a relationship between stress and alcohol use qualified by the drinker's age and the time of the stressor. Alcohol and binge drinking appear to be related to the presence of stress for 
college students and the general population, but the onset of the drinking tends to follow the stressor rather than during or in anticipation of a stressful situation (Rabow \& Neuman, 1984; Volpicelli, 1987). Additionally, Aseltine and Gore (2000) noted that as adolescents and young adults age, alcohol use and stress become less connected which may have implications for the reduction of alcohol use and related consequences during the college years.

The literature on the tension reduction hypothesis was closely examined by Greeley and Oei (1999) who summarized the findings of 15 controlled studies. The authors understood the hypothesis to be the use of alcohol as one possible source of relief of tension when the tension could not be alleviated by another means. No study cited conclusive evidence to show that alcohol reduced tension beyond that of the physiological effects of its chemical properties; expecting the alcohol to provide relief did not appear to have an effect. Individual differences of how stress and anxiety are experienced and relieved were highlighted. This summary of the literature offers an explanation of why research has shown that high levels of stress are not met with increasing alcohol consumption (Corcoran \& Parker, 1991). Having high expectancies about alcohol's ability to manage stress and tension does not appear to be effective, and the anticipated positive outcome of drinking will vary depending on the individual.

Cooper, Russell, Skinner, Frone, and Mudar (1992) also surveyed the research on tension reduction with similar mixed results. Excessive drinking was 
related to the presence of stressful situations, but less than $2 \%$ of the variance in alcohol use could be attributed to the actual stressor. The feeling of tension and the subsequent development of negative affect were proposed as the potential contributors to the increasing use of alcohol after stressful situations occur. This hypothesis does not contradict the theory of Lang et al. (1999) who posited that a positive distraction needs to be perceived by the drinker for tension and negative affect to be reduced or the tension and affect could be exacerbated. Cooper et al. also encouraged differences in individual coping abilities to be a focus of future study on alcohol and tension reduction. They also saw the use of avoidant coping strategies for negative affect among men and African-Americans as being significant contributors to alcohol problems.

Avoidance coping. As early as 1969, Bandura wrote about the use of alcohol as an aid for coping with stress and problems in life when individuals do not have the capacity or ability to cope in any other way. In a college student survey conducted by Berkowitz and Perkins (1987), both male and female heavy drinkers described more escapist and avoidant motives for drinking than students who consumed less alcohol. Recent research continues to support Bandura's hypothesis as binge drinking and "drink tossing" (rapid drinking) are positively associated with avoidance coping in college students (Bonin et al., 2000; Clark 1998). 
Avoidant coping strategies involve making active or passive attempts to distract oneself from an aversive event or condition which can include stress, depression, or loneliness (Bonin et al., 2000). There are situations when avoidant coping can be positive such as with the need to escape social pressures to use substances (Schmitz, Oswald, Damin, \& Mattis, 1995). However, the overuse of avoidance can lead to excessive drinking and alcohol-related consequences if alcohol is expected to remove the stressful condition (Cooper et al., 1992; Simpson \& Arroyo, 1998). When individuals have the emotional capacity to confront their life problems directly with other cognitive or behavioral strategies (approach coping), they tend to use less alcohol than those who rely more strongly on avoidance (Cooper, Russell, \& George, 1988). Simpson and Arroyo (1998) added that the use of avoidance coping should be considered a product of the state of individuals rather than be seen as a trait more deeply rooted in their personality. This suggests that if students have psychological or interpersonal deficits to cope with particular situations, they may be tempted to use avoidant coping and alcohol to manage their response, especially given the availability of alcohol on or around college campuses.

Gender issues. Alcohol has been described above as a complex drug that many individuals and college students seek to manage affect and facilitate interpersonal relations, but gender adds another dimension to how alcohol is involved in social and psychological functioning. Although significant life events 
seem to have no bearing on the drinking problems of women (Cooper et al., 1992), several other psychosocial factors warrant some attention. The rate of alcohol consumption in college women has been positively correlated with negative affect, interpersonal problems, loneliness and emotional distress, and with seeking college counseling services (Brennan, Walfish, \& AuBuchon, 1986; Liu \& Kaplan, 1996). Binge drinking in women has also been linked to high interpersonal stress, restrictive eating patterns, and fluctuations in mood states (Bradstock et al., 1988; Turnbull \& Gomberg, 1988), and a higher frequency of drinking was indicative of low self-esteem (Turnbull \& Gomberg, 1988). Recently, an increase in alcohol consumption and related consequences has been found for women who drink to cope following days of heightened feelings of sadness as compared to men (Hussong, 2007). It appears evident from these studies that excessive alcohol consumption may be serving an important psychological and/or interpersonal purpose for women.

College women state more than men that facilitating socialization and reducing tension are common reasons for drinking, but the drinking is not for relief in social contexts (Berkowitz \& Perkins, 1987). Liu and Kaplan (1996) believed that women consume alcohol for self-medication reasons when encountering depressive symptoms which actually seem to be somewhat reduced by drinking. Baer (2002) reviewed literature on individual differences and alcohol problems and found women consistently reporting the management of negative 
affect and relief of stress as their intentions for using alcohol. A question arises as to why alcohol is given such a critical role in the social lives and mental health of women who drink heavily, but the answer may lie how women differ in their emotional capacity to manage their personal problems and relationships.

Simpson and Arroyo (1998) observed that women who present with alcohol problems appear to have avoidant coping strategies to manage stressful situations as compared to women with no alcohol problems. Women with alcoholrelated consequences typically use avoidance to cope at school/work and a combination of avoidance and acceptance of blame/responsibility to cope in their personal lives. It was proposed that differences in coping with stress would exist between women with alcohol problems and those without problems. The authors noted earlier research that found men to use acceptance of responsibility coping more at work/school than in their personal lives. Acceptance of responsibility was suggested to be a possible means of internalizing affect for women.

For young adult males, alcohol and other substances are often viewed as the path to sensation seeking, social bonding, or fulfilling a need of selfimportance, and an increase in a feeling of power is often reported following use (Liu \& Kaplan, 1996). Capraro (2000) cited several studies to support the view that alcohol use among college men should also be examined in the context of several social and psychological factors, such as depression, gender-role conflict and how the experiences of shame, fear, and power are processed by men. 
Similar to female heavy drinkers, higher consumption rates among men are more likely to be associated with escapist or avoidance motives for drinking, along with high positive expectancies about alcohol producing a desired effect, such as a sense of power or relief (Berkowitz \& Perkins, 1987; Cooper et al., 1992; Poikolainen et al., 2001). Glynn et al. (1983) added that men with alcohol problems use alcohol for socialization and managing negative affect more than men without problems. Murphy, Hoyme, Colby, and Borsari (2006) and Vik et al. (2000) corroborated these results by showing how men expect alcohol to improve their socialization, interpersonal feelings, and overall sense of social comfort; male and female subjects who were identified as having authority problems shared these same expectations. College men, in general, tend to seek more negative experiences and behaviors related to alcohol than women. They will more often have intentions to become intoxicated by alcohol and to feel more aggression when drinking which becomes evident through inappropriate social acts (Berkowitz \& Perkins, 1987).

Conclusions about college drinking. It is apparent that alcohol is desired by young adults for a variety of reasons associated with psychological and interpersonal factors, but the dynamics are complex and potentially damaging to those who rely on alcohol to cope. Not only does alcohol provide an inadequate and inconsistent tool for managing affect and interpersonal relations, but it may magnify the psychosocial problems that already exist in the student and promote 
consequences (Bailey \& Taylor, 1991; Lang et al., 1999). The study of individual differences in emotional and interpersonal functioning has been suggested as a direction for future research on college drinking and alcohol-related consequences. The problem of college drinking is multidimensional and appears to reflect more than just a product of immaturity or peer pressure in students. Understanding deficits in EI may clarify what types of individual differences should be devoted greater attention.

\section{Emotional Functioning, Behavior and Alcohol}

As alcohol research evolved, there have been an increasing number of hypotheses and studies that address the emotional and interpersonal dimensions of binge drinking beyond the physiological effects of alcohol. At the same time, the literature on how deficits in affect management can lead to pervasive psychosocial and physiological problems has also been growing. Studies intersecting these areas of research have been relatively few, especially regarding alcohol-related consequences. However, there appears to be sufficient evidence to justify a greater focus on the relationships between affect and interpersonal management and alcohol problems. Taylor (1997) cited research to support a proposal that alcohol and other substance abuse disorders could be redefined as disorders of affect regulation, noting the potential complicating genetic, personality, and environmental features of this relationship. Constructs such as alexithymia and, more generally, EI have been used as valid means to measure 
and understand affect and interpersonal problems, and a few studies have begun to emerge on their contribution to alcohol problems.

Alexithymia. In 1973, Sifneos coined the term "alexithymia" (meaning "no words for feelings") to describe a pattern of personality characteristics in somatically ill patients. These patients were observed to have an impaired ability to verbally communicate feelings, a lacking or impoverished fantasy life (daydreaming) that was devoid of feelings, and an external mode of thinking. The following characteristics have been added to the description of alexithymia: speech and thought that are concrete, mundane, and closely tied to external events; descriptions of physical symptoms are more common than emotions; a difficulty recognizing inner affect or bodily sensations; and an inability to use these feelings and sensations as signals of emotional distress (Lesser, 1985).

Parker and Taylor (1997) theorized that alexithymia may hinder the development of self-understanding and the ability to manage affect and mood states. Thus, emotional and cognitive deficits may be present that could lead an individual to seek alternative means (i.e., alcohol) to cope with uncomfortable or confusing affect. There has been some support for the hypothesis that alexithymia and emotional inhibition can negatively impact coping. Individuals with alexithymia have been shown to have a paucity of coping responses to stress (Fukunishi \& Rahe, 1995), and the suppression of emotional thoughts has been related to increased autonomic arousal that could impair the process of feeling 
relief in stressful situations (Gross \& Levenson, 1993). Fukunishi (1994) assessed Japanese college students with alexithymia and saw that those who expressed higher levels of hostility and possessed low scores on social desirability were more likely to have poor communication of affect. A survey of college students in the United States yielded similar results when subjects who had high alexithymia were found to be associated with more interpersonal avoidance and nonverbal anger than those with low alexithymia (Berenbaum \& Irvin, 1996).

The connection between ineffective coping strategies and excessive alcohol use (e.g., Cooper et al., 1988) presents the potential for alcohol problems to be related to alexithymia as well. Kauhanen, Julkunen, and Salonen (1992) showed that alexithymia scores increased with the frequency of alcohol intoxication and reports of negative alcohol-related consequences in male subjects. Helmers and Mente (1999) conducted a survey of young, healthy males and examined the relationship between alexithymia and high-risk behaviors. Subjects with low scores on emotional labeling were more likely to have greater alcohol and substance use, but unhealthy sexual behavior was not correlated with alexithymia.

Alexithymia has also been associated with alcoholism and substance abuse in a number of studies (Rybakowski, Ziolkowski, Zasdzka, \& Brezinski, 1988). For example, Ziolkowski, Gruss, and Rybakowski (1995) assessed male outpatients with alcohol dependence and found alexithymia to be present in $48 \%$ 
of the subjects. Subjects who had a duration of abstinence of less than 1 year had significantly higher alexithymia scores than those who had more than 1 year of abstinence. Similarly, alexithymia was found to be a significant predictor of relapse for alcohol-dependent inpatients (Loas, Fremaux, Otmani, Lecerle, \& Delahousse, 1997). Taylor, Parker, and Bagby (1990) led an investigation of the prevalence of alexithymia among newly-abstinent, male, substance abuse patients; $50 \%$ of the subjects were assessed to have alexithymia. Alexithymia was coincident with significantly less ego strength (overall psychological adjustment) and more general dysphoria. Low ego strength and alexithymia may be important factors not only for substance abuse but also college adjustment. Alexithymia and difficulty managing affect have been positively correlated with college maladjustment on the MMPI-II when low ego strength was factored into the model (Fukunishi, 1996).

Several other relationships have been observed between alexithymia and psychological characteristics that tend to remain constant within the individual, in addition to ego strength. These observations have led to growing support for the construct to be considered a stable personality trait, rather than a consequence of psychological distress (Bagby \& Taylor, 1997). Alexithymia, with its stable nature and central focus on affect labeling and emotional self-awareness, shares several conceptual similarities with EI (Taylor \& Bagby, 2000). Parker, Taylor, and Bagby (2001) saw the similarities and used the Bar-On Emotional Quotient 
Inventory and the Twenty-Item Toronto Alexithymia Scale to assess EI. They determined that alexithymia and EI are independent constructs which have a strong inverse relationship. In contrast to alexithymia, EI has the added advantage of incorporating several other emotional and interpersonal variables which are highly desirable for the purposes of this study.

Emotional intelligence as a construct. The recent surge in popularity of EI has led to an inaccurate perception that the construct represents a new field of study. In actuality, the research and theory preceding the development and labeling of EI has occurred over 80 years, beginning with Thorndike's (1920) work on social intelligence (Bar-On \& Parker, 2000; Zirkel, 2000). Social intelligence was proposed as a means to describe how well individuals can selfreflect and understand their behavior as they pursue social and life goals (Zirkel, 2000). The concept of psychological mindedness contributed another aspect of EI because of its emphasis on affect and behavioral management through selfawareness. This area of study centered on the capacity of an individual to understand and reflect on her or his behavior and experience by making connections among thoughts, feelings, and actions (Applebaum, 1973). Parker (2001) believes that EI also stems from an integration of research on ego strength, need for cognition, and private self-consciousness.

Much of the media attention surrounding EI is attributed to Goleman's (1995) best-selling book, Emotional Intelligence, in which the use of term 
"intelligence" generated curiosity and debate. The focus on EI heightened as the construct was marketed as being more valuable to individual functioning than standard intelligence (IQ). It is important to note that EI has been determined to have insignificant relationship with standard cognitive intelligence and should be considered separate constructs (Ciarrochi, Chan, \& Caputi, 2000; Derksen, Kramer, \& Katzko, 2002). The independence of the constructs was corroborated by Morand (1999) who observed that family size was negatively correlated with standard intelligence but positively correlated with EI.

The idea of multiple intelligences (some of which are now essential components of EI) can be mostly credited to a theory proposed by Gardner (1983). Gardner stated that individuals have other forms of intelligence beyond that of cognitive abilities. "Interpersonal intelligence," the potential or ability to understand the emotions and moods of others (i.e., empathy), and "intrapersonal intelligence," the capacity to sense and identify the full range of affect in oneself, were thought to be critical aspects of the individual that affect her or his functioning. Recently, Gardner (1999) expressed some reservation about using an "intelligence" model for EI due to the belief that access to emotion was immersed in both self and interpersonal awareness. Despite theoretical differences and the popularity of EI blurring its intended meaning, other researchers have refined the construct to the extent that it has been influential on the study of psychological and interpersonal behavior. 
While attention was being drawn to Goleman's book, a body of literature began to empirically support the construct of EI as it evolved, and valid assessments were constructed. Goleman's initial definition of EI involved five main domains: self-awareness of one's emotions, the ability to manage emotions, self-motivation and self-control, an empathic understanding of others' emotions, and management of interpersonal relations. Goleman (1998) later expanded his definition to include 25 emotional competencies, such as political awareness and service orientation. These definitions have been criticized for covering too broad of an area which appears to incorporate most psychological variables other than traditional cognitive intelligence (Hedlund \& Sternberg, 2001). Essentially, the breadth of the definition could account for most of the variance when studying individuals in various contexts.

Mayer (1999) concurred with the criticism of Goleman's definitions and expressed concern about the potential overlap with many personality characteristics and the stretching of the construct to predict future life outcomes. As a result, Mayer offered a frequently cited, scientific definition of EI: the capacity to reason with emotion which involves perception, cognitive labeling and integration, understanding emotion, and affect management. The author indicated that this definition of EI can be reliably measured and has been used to support EI as an independent construct from standard intelligence, though some relationship has been demonstrated. 
Bar-On (1997/2002) viewed EI as a multidimensional construct of noncognitive abilities that can be organized into 5 measurable components: personal or intrapersonal (which includes emotional awareness, assertiveness, self-regard, self-actualization, and independence), interpersonal (empathy, interpersonal relationships, social responsibility), adaptability (problem solving and reality testing), stress management (stress tolerance, impulse control), and general mood (happiness, optimism). This description of EI was also criticized by Mayer (2001) for the exclusion of emotional reasoning and for being too inclusive of personality traits. However, Hedlund and Sternberg (2001) reported that over 15 different measures have validated Bar-On's formulation of EI as assessed by the BarOn Emotional Quotient Inventory (EQ-I; Bar-On, 1997/2002).

The debate over the theoretical details of EI continues, but it has not interfered with the construct being accepted as useful and valid. Martinez-Pons (1997) offered support for the construct validity of EI and found it to be positively related to subjects' goal orientation and life satisfaction. High EI was also negatively correlated with depression symptomatology. Ciarrochi et al. (2000) looked closely at the validity of EI and found that the construct was correlated with some measures of personality (e.g., empathy), life satisfaction, and mood management. The authors observed that while EI reflects an individual's ability to manage and change mood states, it does not appear to have a mediating effect on the judgments that arise during the various mood states. This interpretation of the 
data suggests there could be potential problems for an individual with low EI who chooses to binge drink. It is not known if the intoxicating properties of alcohol could exacerbate poor mood management, encourage negative mood states, or lead to uninhibited poor judgments and consequences that result from binge drinking.

Forgas (2001) emphasized the critical role of affect and mood in decision making, judgment, and behavior and how EI could be seen in this process. Behaviors that are enacted without conscious thought or self-awareness are subject to the influence of the affect present at that moment. High EI would allow individuals the ability to examine a situation comprehensively, incorporating multiple emotional and interpersonal variables into the most beneficial decision. The capacity to access and be aware of emotional cues and mood states with accuracy can enable greater management of situational affect in a broad array of contexts (Salovey, Bedell, Detweiler, \& Mayer, 1999). Individuals who exhibit a greater capacity for adapting to change, emotional self-regulation, and reduced social comparison have been found to be related to reductions in heavy drinking among college students (Carey, Henson, Carey, \& Maisto, 2007).

Matthews and Zeidner (2001) described the hypothesis underlying EI with an emphasis on the cognitive and emotional assets individuals have developed to confront emotionally provoking situations. They proposed that deficits in EI may make one vulnerable to poor decisions and risky behaviors that could lead to 
substance abuse, physiological problems and/or emotional distress. This theory is consistent with the literature on the relationships that exist among the excessive use of alcohol, poor emotional coping and interpersonal skills, and alcohol-related consequences.

Consequences of low emotional intelligence. According to Lazarus (1991), emotion and mood factors may have a critical role in an individual's behavior by encouraging either appropriate or inappropriate actions to a given situation. Aggression would be an example of one possible response to a sensation of anger that could lead to negative consequences. What causes individuals to engage in harmful behaviors continues to be debated, but the EI construct may provide an effective framework for understanding these consequences.

The recent development of EI has limited the number of studies and hypotheses specifically addressing the effects of low EI. Initial theoretical considerations suggest that low EI and deficits in managing affect would lead to maladaptive coping strategies and subsequent problems, such as binge drinking (Matthews \& Zeidner, 2001; Salovey et al., 1999). The research on high alexithymia, noted previously, encourages this direction by demonstrating that emotional labeling deficits are associated with alcohol-related consequences, interpersonal problems, and low ego-strength.

Studies on violence and aggression have also shown promising links to EI or one of its components. Block (1995) found that impulsivity in pre-adolescents 
was a better predictor of behavioral problems than verbal intelligence. Police officers who were able to calmly manage emotionally provocative situations with angry motorists recorded fewer incidents escalating to violence (Brondolo, 1996). When EI was specifically measured, high scores were indicative of fewer problems with violence and other inappropriate behavior, as gender, standard intelligence and empathy were being statistically controlled (Cobb \& Mayer, 2000). A similar result was noted by Mayer, Caruso, and Salovey (2001) who cited some of their own unpublished raw data on college students. High scores on the Multifactor EI Scale (MEIS) were negatively correlated with self-reports of violent and trouble-prone behavior after standard intelligence and empathy were partialled out.

Of particular relevance to the present study, low scores in emotional intelligence in adults have been found to be a predictor of alcohol use and alcohol-related problems (Riley \& Shutte, 2003; Trinidad \& Johnson, 2002). Another survey concerning alcohol use and EI was conducted on behalf of the United States Air Force. Sitarenios and Handley (1998) assessed the EI (using the BarOn EQ-i) of military personnel with various levels of alcohol problems. Subjects who abuse alcohol had lower scores on the problem solving, stress tolerance, and self-regard components of the EQ-I, but the results were not significant when compared to scores from the normal population of military 
personnel. It should be noted that the authors questioned the statistical power of these results due to the relatively small sample size of the alcohol abuse group.

The combination of emotional deficits and binge drinking is likely to heighten the chances of an individual making poor personal and interpersonal decisions. Frequent binge drinking has been associated with disinhibition and the use of alcohol to cope with stress and uncomfortable affect (Ichiyama \& Kruse, 1998). Furthermore, college students with the most damaging alcohol-related consequences consume the largest quantity of alcohol and tend to expect alcohol to improve their socialization, reduce their interpersonal distress, and increase positive affect (Vik et al., 2000). How college students with low EI are impacted by binge drinking is not yet known, but it appears that many who fall into this group may be susceptible to the negative emotional and interpersonal consequences of excessive alcohol use.

\section{Summary and Research Questions}

Thousands of college students are injured, abused, and killed each year from incidents involving excessive alcohol use. Colleges spend a significant amount of money repairing property damage, hiring security and alcohol counseling staff, and implementing alcohol prevention programs in an attempt to reverse the trend of increasing consequences. In addition to these more visible consequences are the overwhelming number of students who experience problems in their academic performance, interpersonal relationships, and mental/physical 
health due to alcohol. The enormous costs associated with college binge drinking have led to a plethora of research intending to better comprehend and combat the problem. The prevention efforts that have been implemented appear to have had minimal impact on reducing binge drinking consequences, but there have been some promising results from wellness-oriented programming.

Alcohol and prevention research has recently begun to contradict the view that excessive drinking is the only common denominator for alcohol-related consequences. More attention is being devoted to individual differences and psychological factors surrounding alcohol consumption. Baer (2002) emphasized the need to study a variety of drinking motives for college students who come from all facets of the college community. Targeting prevention efforts to help sociopathic students who frequently drink in high excess will lead to a number of students with alcohol problems being missed. Consequences from binge drinking are present for nonbinge drinkers as well and need to be studied apart from the view that problems are exclusively a by-product of alcohol intoxication (Caldwell, 2002). As more contributing factors to binge drinking and alcoholrelated consequences are identified, alternative prevention programs can be created to address the many dimensions of alcohol abuse.

One new direction of research on college alcohol use has led to the understanding that binge drinking can be a meaningful activity for many students. This is especially true for those students who may be seeking new socialization 
opportunities or who are feeling inadequate in their ability to manage relationships or affect (Maggs, 1997; Mooney, et al., 1987). Studies have shown that excessive alcohol use is connected to a desire to improve socialization, a need to reduce tension, and an inability to cope effectively with uncomfortable affect and moods. However, relatively few research efforts have been conducted to explore how alcohol-related consequences alone may be indicative of an emotional or interpersonal need or deficit. Empirical evidence demonstrates how alcohol is often used as a coping mechanism, as Bandura (1969) proposed, but the literature also seems to suggest that alcohol-related consequences may be expressions or reflections of psychological deficits that become more apparent with alcohol intoxication.

Alexithymia and EI are broad constructs that help to define emotional functioning and to describe possible affect- and interpersonal-oriented limitations. In particular, Bar-On's (1997/2002) formulation of EI provides a useful framework for measuring an individual's ability and weaknesses on a number of emotional and interpersonal variables. Subjects assessed to have high EI typically describe higher life satisfaction, and those with high alexithymia tend to report psychological distress, frequent excessive drinking, and alcohol-related consequences. Studies of psychological factors related to these constructs also have found that emotional and interpersonal deficits are frequent precursors to alcohol and other life problems. Due to the recent evolution of the EI construct, 
there is limited research on problems associated with low EI, although depression symptomatology and inappropriate aggressive behaviors have been related. Additionally, several theorists promote the view that low EI would indicate psychological vulnerabilities in individuals, potentially leading to problems with interpersonal judgment, alcohol abuse, or other ineffective coping behaviors.

College students who are young adults are in the midst of a challenging developmental transition in their life because they often are leaving their primary social support system and entering into an academically and socially stressful environment. Their youth combined with any possible deficits in psychological functioning or EI could overtax their ability to cope. The availability of alcohol and the social pressure to drink may promote the use of alcohol as a coping mechanism or social catalyst, potentially setting the student up for negative consequences. Many healthy students engage in binge drinking without serious consequences, but some drinkers seem to be more prone to encounter negative experiences from drinking episodes. The use of a construct like EI may help to explain these individual differences that occur with regard to binge drinking and alcohol-related consequences. Revealing the associations that exist among select EI components and specific alcohol-related consequence categories would be a valuable contribution to alcohol theory and prevention research.

A review of the literature pertaining to college binge drinking and psychological functioning offers theoretical and indirect empirical support for the 
relationship between alcohol-related consequences and EI. Based on this review, the following research questions are proposed for the present study:

1) Do college binge drinkers and non-binge drinkers differ on a measure of emotional intelligence (EI)?

2) Do binge drinkers with consequences differ from binge drinkers without consequences on a measure of EI?

3) Are there emotional and interpersonal components of EI that predict different types of alcohol-related consequences across binge drinking college students?

4) If significant relationships exist among EI factors and specific types of alcohol-related consequences, are there any variations across demographic variables? 


\section{CHAPTER THREE}

\section{Method}

In the following chapter, the methods involved in conducting the proposed study are discussed with regard to the participants, measures, procedure, and statistical analyses used to address the proposed research questions. Demographic descriptive statistics are provided in the results section.

\section{Participants}

A convenience sample of 309 participants were surveyed in the present study as undergraduate student volunteers solicited from two small, liberal arts colleges in the northeastern United States and one large, urban Midwestern university. They were recruited from undergraduate introductory and advanced courses in psychology and education during the period of February 27, 2006 to April 10, 2007. They received extra academic credit for full participation in the study at the discretion of the instructor, and their names were placed in a random drawing for a $\$ 100$ cash prize. Information regarding the participants' age, gender, education, socioeconomic status, ethnicity, class year, Greek system membership, and intercollegiate athletic participation was collected via the Research Questionnaire. Participants who returned incomplete assessments were excluded from all data analyses.

Data from 15 participants were discarded due to incomplete surveys, invalid assessments or voluntary withdrawal from the study. Of the 309 
completed sets of assessments, female participants (198) almost doubled the number of male participants (111). The overwhelming majority of participants were Caucasian (88.7\%), and those in the 18-20 (47.2\%) and 21-23 (41.1\%) age ranges were most represented. The class with the highest percentage of participants was seniors (35.9\%) with the lowest being first-year students (17.8\%). Intercollegiate athletes and members of Greek systems made up 19.1\% and $16.8 \%$ of the participants, respectively.

\section{Measures}

The following will provide an overview of select nonstandardized and standardized measures that pertain to each of the main variables of this study. The measures were chosen based on their frequency of use in the literature, empirical support, and/or relevance to the current study.

Binge drinking measures. Numerous alcohol consumption assessments have been developed for the past several decades for the purposes of research, diagnosis, and clinical data gathering. The designs of the instruments have largely been dependent on the desired data for collection and on the operational definitions of alcohol dependence, alcohol abuse, and binge drinking. Some of the early popular measures of alcohol use attempted to identify alcohol patterns and problems by calculating and categorizing consumption. Information on an individual's quantity and frequency of alcohol consumption is consolidated for specified time periods and across different contexts (e.g., alone vs. drinking with 
friends) yielding scores to classify drinking problems. For example, the QuantityFrequency Variability Index (QFV; Cahalan, Cisin, \& Crossley, 1969) is a selfadministered survey of an individual's quantity and frequency of alcohol consumption in the previous 4-6 week period; type of alcohol (i.e., beer, wine or liquor) and social context are also considered. The results yield high-risk drinking patterns, but the classification system and categorical data are relatively complicated (Sobell \& Sobell, 1995).

Other drinking measures aid in the diagnosis of alcohol abuse and dependence or have been designed for research that intended to assess alcohol problems without detailed consumption rates. The CAGE Questionnaire (Mayfield, McLeod, \& Hall, 1974) is a simple, 4-question screening tool administered by a clinician to detect alcohol dependence. It has been found to be a reliable and valid instrument for identifying alcohol problems (Chan, Pristach, Welte, 1994). Another frequently cited measure that has several modified versions is the Michigan Alcoholism Screening Test (MAST; Selzer, 1971). The original instrument is a 25 -item, self- or clinician-administered test of lifetime alcohol problems and potential alcohol dependence. The questions revolve around general alcohol use, drinking contexts, and alcohol-related consequences. Studies have demonstrated adequate reliability and validity for the measure (Hedlund \& Vieweg, 1984; Skinner \& Sheu, 1982), but the questions regarding consequences appear to be relatively few and inappropriate for a college population. For both 
the MAST and the CAGE, the data generated on quantity and frequency of consumption are nonspecific which becomes problematic when attempting to identify binge drinking.

The Alcohol Use Disorders Identification Test (AUDIT; Babor, de la Fuente, Saunders, \& Grant, 1992) is published by the World Health Organization and has become a commonly used instrument in healthcare screenings and epidemiological studies. It is a 10-item, self-report measure of hazardous drinking; 3 of the test items address details of the participant's drinking rate while 4 items target alcohol-related consequences. Validation of the AUDIT has been established by high correlations with the CAGE ( $r=.78$; Hays, Merz, \& Nicholas, $1995)$ and with the MAST across gender differences $(r=.88$; Bohn, Babor, \& Kranzler, 1995). High correlations also have been reported between the AUDIT and measures of drinking consequences, drinking attitudes, alcohol-induced negative mood states, and reasons for drinking (Hays et al., 1995). The AUDIT has been shown to predict unemployment over a two year period (Claussen \& Aasland, 1993) and the onset of physical disorders, alcohol-related social problems, health care utilization, and hazardous drinking risks (Conigrave, Saunders, \& Reznik, 1995). Sinclair, McRee, and Babor (1992) examined the testretest reliability of the AUDIT among drinkers with different levels of alcohol use and found a strong correlation of $r=.86$. 
Babor, Higgins-Biddle, Saunders, and Monteiro (2001) describe the AUDIT as a tool that can accurately discern an individual's drinking level across a wide range of severity in alcohol consumption. In particular, it has been supported for use with the college student population (Fleming, Barry, \& MacDonald, 1991). Although there are available norms for heavy drinkers and alcohol dependent populations, the quantity and frequency of consumption items are not compatible with the gender-specific, binge drinking criteria established by Wechsler, Dowdall, Davenport, and Rimm (1994).

One of the most significant developments in the area of alcohol use assessment has been the proposal of the "five/four measure" as the criteria for binge drinking. Wechsler, Davenport, et al. (1994) observed in their 1993 national survey of college student drinking that the number of alcohol-related consequences tended to markedly increase after a specific amount of alcohol. In particular, the number of social consequences attributed to alcohol appeared to grow substantially when men consumed 5 or more drinks and women consumed 4 or more drinks in a row during the 2-week period prior to the survey. Additionally, several other researchers have determined that having 5 or more drinks on one occasion was significantly associated with social harm (Cahalan, Cisin, \& Crossley, 1969), drunk driving, employment problems, and alcohol dependency (Midanik, Tam, Greenfield, \& Caetano, 1996). Presley, Meilman, and Lyerla (1995) indicated that the 5 drink standard is appropriate for determining 
college binge drinking, but gender differences need to be taken into account. By lowering the 5 drink standard to 4 drinks for women, the risk of alcohol-related consequences becomes more balanced across genders (Wechsler, Dowdall, et al., 1994).

The support for the five/four measure has led many researchers to use this criterion as an operational definition of binge drinking for the process of developing their own questionnaires and surveys (e.g., Camatta \& Nagoshi, 1995; Wechsler, Lee, Kuo, et al., 2002). Concerns about these and other nonstandardized measures of alcohol consumption have existed in the literature due to questions about the veracity of self-reports of past alcohol use. However, some research has shown that retrospective self-reports of alcohol consumption can offer comparable data to other measures.

Lemmens, Tan, and Knibbe (1992) studied differences among 5 general self-report methods of alcohol consumption: a prospective diary, a retrospective 7-day recall method, and 3 quantity-frequency summary methods (past 6 months, variability within the 6 months, and total usual daily consumption). The diary was observed to have the highest coverage rate $(67 \%)$ of the total alcohol sales estimates in the region, suggesting that the diary method captured approximately $67 \%$ of the alcohol sold. A simple quantity-frequency index had a coverage rate of $46 \%$ and a rank order correlation of .61 with the diary method. There was minimal difference found for heavy drinkers with steady consumption patterns in 
drinking reported by the diary versus the quantity-frequency summary methods. Underreporting of the frequency of drinking episodes was more common than the underreporting of the number of drinks, and underreporting, in general, could be mostly attributed to forgetting. Shakeshaft, Bowman, and Sanson-Fisher (1998) conducted a similar investigation by comparing 3 methods of assessing binge drinking: the AUDIT, a one-week retrospective diary, and a quantity-frequency measure. They found that the AUDIT was able to distinguish the most binge drinkers, but the 3 methods generally demonstrated adequate agreement with each other.

Many alcohol and binge drinking assessments have been created or customized to meet the needs of researchers with satisfactory results. In the present study, three self-report questions were developed to quantify alcohol use patterns and identify binge drinking in accordance with the "five/four measure" of binge drinking proposed by Wechsler, Davenport et al. (1994). The questions are taken directly from or modeled after the first 3 items that assess drinking rate in the AUDIT, due to the AUDIT's empirical support for use with college students (Fleming et al., 1991). Similarly, Shakeshaft, Bowman, and Sanson-Fisher (1999) compared the first 2 items of the AUDIT with a weekly retrospective diary. Higher consumption patterns and high-risk drinking were more readily seen via the diary but not when the comparisons were conducted based on gender; there were no statistically significant differences in the identification of high-risk 
drinkers for women. The combined score of the two AUDIT items were highly correlated with the diary (.63). Additionally, the order and wording of AUDIT questions have been successfully modified in a previous study by Ivis, Adliaf, and Rehm (2000) with no effect on scoring or internal consistency reliability.

The Research Questionnaire was developed for the present study to collect demographic data and information pertaining to frequency and quantity of alcohol use in order to determine binge drinking status. Demographic data are solicited for age, gender, ethnicity, college year, socioeconomic status intercollegiate athletics participation, and Greek system membership. There are three items on the questionnaire that use a 5-point or 6-point Likert-type scale to assess alcohol consumption and binge drinking. The items that request the number of alcoholic drinks consumed within the past two weeks were taken or adapted from the AUDIT. The first question asks the participant to report frequency of alcohol consumption from "Never" to "Four or more times per week." The second question assesses the number of binge drinking episodes within the past two weeks from " 1 or 2 " to "More than 5." The third question addresses the frequency of binge drinking from "Never" to "Daily or almost daily," based on the finding that frequency is associated with coping, disinhibition, and high risk sexual activity for men (Ichiyama \& Kruse, 1998). Binge drinking is operationally defined as an endorsement of four or more drinks of alcohol in one drinking episode within a two week period for females and an endorsement of five or more 
alcohol drinks in one episode within a two week period for males. See the Research Questionnaire in Appendix B for the exact questions used to assess demographic status and binge drinking in the present study.

Alcohol-related consequence measures. As with many of the alcohol consumption measures, assessments of alcohol-related consequences are often designed by the authors for a specific study. Researchers frequently use the alcohol consequences reported in national surveys and the literature to develop a list of items that suit their intended data collection (e.g., Camatta \& Nagoshi, 1995; Cronin, 1997; Wechsler et al., 1998). Another psychometric technique has been to generate an abbreviated or modified version of a more comprehensive, standardized alcohol assessment tool (e.g., Vik et al., 2000). Regardless of how the measure is created, the items or consequences endorsed are typically summarized in percentages by item, and/or a total number of consequences attributed alcohol is tallied for each individual (e.g., Ichiyama \& Kruse, 1998; Wechsler et al., 1998). The consequences are rarely broken down into categories. Three standardized measures of alcohol-related consequences have been developed for use with adolescents and young adults, including college students. The Student Alcohol Questionnaire and Alcohol Attitude Questionnaire (Engs \& Hanson, 1981) is a comprehensive alcohol assessment that examines and summarizes demographic data, alcohol consumption rates, attitudes and expectancies, and alcohol-related consequences (17 items). Criterion and content 
validity has been established, and internal reliability was found to be adequate at .79. The Rutgers Alcohol Problem Index (RAPI; White \& Labouvie, 1989) is a 23-item, self-report measure of lifetime problems due to alcohol use for adolescents between the ages of 12 and 21 . The results identify consequences that have occurred during and after drinking episodes. Carey and Correia (1997) supported the use of the RAPI with college students and noted a strong internal consistency of .85 , only somewhat lower than the internal consistency of .92 reported by White and Labouvie. Hurlburt and Sher (1992) designed the Young Adult Alcohol Problems Screening Test (YAAPST) to specifically assess binge drinking consequences among college students. It has now evolved into a 36-item, self-report measure, but the authors determined that it was a highly reliable and valid instrument in its original 27-item form. Although the YAAPST can yield descriptive data on recency and severity of consequences, it doesn't, nor do any of the above assessments, provide categories to differentiate type of consequence. LaBrie, Pedersen, and Tawalbeh (2007) recommended that alcohol problems be assessed in categories and beyond a 2-week period of time to more accurately represent the individual's drinking habits and consequences.

The Alcohol Use Inventory (AUI; Wanberg, Horn, \& Foster, 1977) and the Drinker Inventory of Consequences (DrInC; Miller et al., 1995) are two standardized instruments that allow alcohol-related consequence data to be categorized. The AUI is a 228-item, comprehensive, self-report measure of 
alcohol use problems that provides 24 scales of alcohol consumption patterns, expectancies, drinking contexts and types of problems. The consequence scales include: loss of control, role maladaptation, delirium, hangover, and marital problems. Reliability and validity (content, construct, and criterion) studies have determined that the AUI is a psychometrically sound instrument (Horn, Wanberg, \& Foster, 1987; Isenhart, 1990).

In contrast to the AUI, the DrInC is a 50-item, self-report measure of lifetime consequences from alcohol abuse that also excludes questions related to dependency and treatment. The sole purpose of the DrInC is to organize alcoholrelated consequence data into five categories: physical, intrapersonal, social responsibility, interpersonal, and impulse control. The physical subscale represents the acute and chronic adverse physical effects of excessive alcohol use, including injury and physiological and sexual problems; there are eight items that make up this subscale which ranges from 0 to 8 . The intrapersonal subscale looks at alcohol-related consequences that are internal to an individual regarding their personality, personal growth, spirituality/morality, interests, and self-esteem; there are eight items that make up this subscale which ranges from 0 to 8 . The social responsibility subscale measures problems regarding role-fulfillment with respect to work, school, financial management, and other personal expectations; there are seven items that make up this subscale which ranges from 0 to 7 . The interpersonal subscale examines the consequences affecting interpersonal 
relationships, such as loss of close friends/romantic partners, harm to family, increased concern from significant others regarding drinking, and embarrassment; there are 10 items that make up this subscale which ranges from 0 to 10 . The last category is the impulse control subscale which addresses increased risk taking behaviors, accidents, causing damage to property or others, police action, and the broadening of use to other substances; there are 12 items that make up this subscale which ranges from 0 to 12 .

An initial factor analysis was conducted on the test items by Tonigan and Miller (1993) which yielded four factors (legal, social, intimate, and intrapsychic consequences) with $30.2 \%$ of the item variance being accounted for by these factors. Miller et al. (1995) reported that the DrInC exhibited a test-retest reliability cofficient of .93 and an internal consistency coefficient of .91 for the total number of consequences; see Table 1 in Appendix D for a summary of the psychometric support. Convergent validity was established through positive correlations with a number of alcohol and psychological measures, including the Beck Depression Inventory $(r=.26)$, an alcohol consumption assessment $(r=$ .40 ), and the G scale (focusing on alcohol consequences; $r=.61$ ) of the AUI (Miller et al., 1995; Tonigan \& Miller, 1993). Recently, Simpson and Arroyo (1998) used the DrInC on a sample of female college students and stated that the test possessed several items that demonstrated face validity for using the measure with women, such as items regarding problems with parenting or close 
relationships. The focus on only binge drinking consequence data (that correspond well to the EQ-i) and the lack of dependency or treatment items are the primary reasons for using the DrInC in the present study. See Appendix C for a copy of the DrInC.

Emotional intelligence measures. Shortly after the development of the EI construct, several instruments were designed to provide a vehicle for individuals to quantify and reflect on their own emotional competencies. Corporations also capitalized on these new tools in an attempt to have greater access to the emotional functioning of their employees and applicants. Other "tests" included self-report measures constructed without any scientific basis or technique for primarily entertainment purposes in the media and on the internet (Mayer, Caruso, \& Salovey, 2000). Recently, a few instruments have been empirically tested and validated, but the continued debate over the definition of EI has created some theoretical construct differences among assessments.

One approach has been to focus on EI as a cognitive process or "intelligence" that can be observed by completing a number of affect-related tasks. The Mayer, Salovey, Caruso EI Scale (MSCEIS; Mayer, Salovey, \& Caruso, 1999) assesses the ability of individuals to perceive and think through emotional material and to make healthy interpersonal and emotional responses. The data are categorized into 4 branches (consisting of 12 subscales): emotional perception, emotional facilitation, emotional understanding, and emotional 
management. Internal consistency ranged from .59 to .87 among the EI branches; a full-scale internal consistency score of .96 was attained on the original version of the test (Multifactor EI Scale [MEIS] Mayer et al., 2000). Ciarrochi et al. (2000) supported the validity of the MEIS to measure EI and reported a full-scale internal consistency of .90 .

Most of the other EI assessments were designed to examine EI not as a cognitive ability but as a construct representing a competency level in emotional and interpersonal functioning. The Emotional Competence Inventory 360 (ECI: Boyatzis, Goleman, \& Hay/McBer, 1999) is the only measure of EI that involves both self-report and observer ratings in the data collection. The ECI measures 20 competencies that relate to emotional and interpersonal awareness, motivation, and affect management; the competencies are divided into 4 categories: selfawareness, social awareness, self-management, and social skills. Sala (2002) indicated that construct, criterion, and content validity have been established and that the ECI could be differentiated from a measure of critical and analytical thinking. The internal consistency coefficient (Chronbach's alpha) was .85 for the observer-rated items and .75 for the self-report items; .61 was the lowest coefficient for any of the 20 competencies.

Two measures have emerged as the dominant, self-report assessments of EI. The EQ-Map (Cooper, 1996/1997) consists of 20 subscales to provide an outline of the emotional functioning of individuals at home and work. The data 
are organized into five categories: current environment, emotional literacy, EQ competencies, EQ values and attitudes, and EQ outcomes. The publishers stress that the EQ-Map is not a "test" and market the instrument to be predominantly used with corporate training and personal self-learning. As a result, the reliability and validity data are described as adequate but are vague and not readily available.

The BarOn Emotional Quotient Inventory (EQ-i; Bar-On, 1997/2002) is a second self-report measure of EI that appears to have the most empirical support and use in the field. Bar-On understands EI as a construct of noncognitive ability to manage emotions, evidenced by personal and interpersonal success with the stress and pressures of daily life. The design of the EQ-i consists of 133 items intended to yield EQ (emotional quotient) scores in five categories (divided into 15 factors): intrapersonal (including emotional self-awareness, assertiveness, selfregard, self-actualization, and independence), interpersonal (empathy, interpersonal relationship, and social responsibility), stress management (stress tolerance and impulse control), adaptability (problem solving, reality testing, and flexibility), and general mood (happiness and optimism). There are also four validity indices and a correction factor. Scores typically range from 55 to 145 on all of the scales and subscales which represent three standard deviations above and below the mean (100). It should be noted that the mean EQ-i score is 95.3 for the 16 to 19 age range and 96.8 for the 20 to 29 age range (Bar-On, 1997/2002). 
The following is a description of the 15 EQ-i subscales (including the number of test items in each subscale) that make up the scores for the five factors and the total EQ-i score: a) the emotional self-awareness subscale (eight items) addresses the ability to label, differentiate, and understand one's own feelings; b) the assertiveness subscale (seven items) examines the ability to express one's own feelings and beliefs using appropriate behavior; c) the self-regard subscale (nine items) looks at the ability to have self-confidence, a positive identity, inner strength, acceptance that one is basically good, and feelings of self-adequacy; d) the self-actualization subscale (nine items) measures the ability to strive towards one's potential capacities in pursuing meaningful activities and long-term goals; e) the independence subscale (seven items) represents the ability to function with self-direction and self-control without emotional dependency; f) the empathy subscale (eight items) assesses the ability to perceive, understand, and demonstrate concern for others' feelings; g) the interpersonal relationship subscale (11 items) addresses the ability to achieve positive and healthy relationships that reflect intimacy, mutual satisfaction, and affection; h) the social responsibility subscale (10 items) examines the ability to be a responsible member of one's social group with acceptance of others and selfless contribution for the good of the group; i) the problem solving subscale (eight items) looks at the ability to perceive, manage, and resolve problems in a thoughtful and systematic manner; j) the reality testing subscale (10 items) measures the ability to judge the 
validity of what is experienced from a clear understanding of the objective evidence; k) the flexibility subscale (eight items) represents the ability to adapt one's feelings, thoughts, and behavior to the diverse and changing environments to which one is exposed; 1) the stress tolerance subscale (nine items) assesses the ability to positive cope with emotionally challenging situations; $\mathrm{m}$ ) the impulse control subscale (nine items) addresses the ability to reflect and pause before acting on impulses or aggressive temptations; $n$ ) the happiness subscale (nine items) examines the ability to live a life of general contentment, satisfaction, and enjoyment; and o) the optimism subscale (eight items) looks at the ability to maintain hope and positive expectations regardless of negative life experiences. Bar-On (1997/2002) reported numerous studies that demonstrate the reliability and validity of the EQ-I; see Table 2 in Appendix D for a summary of the psychometric support. Nine studies noted internal consistency scores ranging from .69 to .86 , and all Item-Total correlations have been observed to be greater than .30 for each factor (Sitarenios, 1998). Bar-On (1997/2002) cited that the average retest reliability coefficient of the EQ-i was .85 at one-month and .75 at four-months.

The construct validity for the EQ-i's composite scales and subscales was assessed by several factor analyses and confirmatory factor analyses which supported a 15-factor structure. Ten assessments measuring various aspects of emotional and interpersonal functioning added to its construct validity with 
correlational coefficients ranging from .30 to .70 . Convergent validity was achieved by positive associations with acculturation, attributional style, ability to cope with occupational stress, job performance, and work satisfaction. In terms of divergent validity, the EQ-i was found to not be a measure of reasoning, aggression, low self-esteem, and emotional instability (The EQ-i measures adaptability.). Pallazza and Bar-On (1995) added that the problem solving subscale of the EQ-i was not related to a measure of cognitive intelligence. BarOn (1997/2002) offered criterion group validity support for the EQ-i by providing data to show significant differences between samples from the Young President's Organization and chronically unemployed persons. Furthermore, scores from samples of psychologists and financial services professionals were significantly different from the normative sample on a number of EQ-i scales. The EQ-i also discriminated within a group of military academy students by their self-reported ability to cope with stress and within a group of individuals who were divided by level of emotional functioning, as determined by the Sixteen Personality Factor Questionnaire (16PF; Cattell, Eber, \& Tatsouka 1970). Predictive validity was observed using the following variables: academic and occupational success, ability to acculturate, and likelihood to benefit from a substance abuse rehabilitation program.

The EQ-i has also received empirical support from independent researchers. Derksen et al. (2002) reported a correlation of .08 between the EQ-i 
and the General Ability Measure for Adults (GAMA; Naglieri \& Bardos, 1997) further distinguishing the EQ-i from being a measure of general intelligence. In contrast, the adaptability and stress management factors of the EQ-i have been shown to be negatively correlated with the Twenty-Item Toronto Alexithymia Scale (TAS-20; Bagby, Parker, \& Taylor, 1994) and its three factors (difficulty identifying feelings, difficulty describing feelings to others, and externally oriented thinking). Dawda and Hart (2000) examined the reliability and validity of the EQ-i against several measures of personality, symptom presentation, and affect/mood, including alexithymia. The authors concluded that a wide array of emotional constructs is assessed by the EQ-i which appeared to be free of gender bias. One concern arose regarding interpersonal factor of the EQ-i which had relatively weak associations with the remaining EQ composite scales and a unique pattern of convergent and discriminant validities. However, the EQ-i's total score was determined to be a good indicator of EI. Hedlund and Sternberg (2001) also stated that the EQ-i appears to be representative of Bar-On's model of EI and supported its use, noting some potential problems of social desirability and susceptibility to faking. Likewise, independent reviewers from the Mental Measurements Yearbook found the EQ-i to have sound psychometric properties and to be a good assessment of EI (Impara \& Plake, 2001). As a result of the above support for Bar-On's formulation of EI and the EQ-i's good reliability and validity data, the present study will use the EQ-i to assess the EI of college 
students. See Appendix B for a copy of the EQ-i’s Item Booklet and Subscale Composition.

Procedure

Participants were recruited on a voluntary basis from undergraduate psychology and education courses at two small, rural, liberal arts colleges in the northeast and one large, urban Midwestern university in order to diversify the sample. Institutional review board (IRB) approval was required and achieved at one college, but it was not necessary for the remaining two. In order to facilitate recruitment, instructors allowed the use of class time, academic credit was offered at the discretion of the instructor, and the names and contact information of willing participants were placed in a raffle for a $\$ 100$ cash prize; the names and contact information were kept separate from the data collected from the assessments.

Prior to the administration of the assessments, all participants heard a brief oral description of the study and then read and signed the Consent and Information Form (see Appendix E). The study was described as a research project designed to assess the psychological factors that may be involved in the consequences of binge drinking. They also were told that their name and any personal information that could identify them would not be required for their assessments or scores in order to protect their anonymity. Accommodations were offered depending on disability status of the participant or other variable that may 
affect the validity of the assessments. No participant requested an accommodation. The instructors were not present during the administration of the assessments in order to avoid any perceived influence on the participants.

Participants were asked to question the experimenter if further clarification of the procedure was needed and then to complete three brief, written assessments (Research Questionnaire, DrInC, and EQ-i) which lasted approximately 25 minutes. The online version of the EQ-i was offered to allow participants greater anonymity, but no participant chose this option. Participants who completed all of the self-report measures were asked for feedback about the assessments to determine if the participant had any difficulty. The examiner followed with a request that the participant fill out an entry form for the raffle. The data obtained from the questionnaire and assessments were collated, entered into a computer program and analyzed by the SPSS statistical software package.

\section{Statistical Analyses}

The current study used both descriptive and inferential statistics to analyze the data. Prior to the statistical analysis of the research questions, t-tests and analyses of variance (ANOVAs) were used to explore any demographic differences that exist for EI, binge drinking status, binge drinking frequency, and binge drinking consequences. The results offered a general picture of the representativeness of the sample and how age, gender, ethnic, undergraduate class, athlete status, greek system membership, and SES status were important 
considerations for EI and binge drinking. Tables will be presented in the results section with the frequency data of each variable measured. The following is the list of the four research questions examined in the present study and the proposed statistical analyses that were intended to be used on the data to yield results for discussion:

1) Do college binge drinkers and non-binge drinkers differ on a measure of emotional intelligence (EI)?

The independent variable for Research Question 1 was "College Binge Drinking Status” (“Binge Drinker” vs. "Non-binge Drinker”); scores of 4 or higher on questions 2 or 3 of the Research Questionnaire indicate a "Binge Drinker." The dependent variables were participants' scores on measures of general emotional intelligence and the five factors of emotional intelligence: intrapersonal, interpersonal, stress management, adaptability, and general mood, as assessed by the BarOn EQ-i.

One MANOVA was used to examine differences within binge drinking status (binge drinker vs. non-binge drinker) on each of the five factors of the EQ-i, and a t-test was conducted to determine if binge drinkers differ from non-binge drinkers on a measure of general emotional intelligence.

2) Do binge drinkers with consequences differ from binge drinkers without consequences on a measure of EI? 
There were two independent variables for Research Question 2: "Binge Drinker Endorsement of Consequences" ("Participants Reporting Consequences" vs. "Participants Reporting No Consequences") and "Type of Consequence" ("Physical," "Interpersonal," "Intrapersonal," "Impulse Control," and "Social Responsibility"). For the binge drinker endorsement of consequence variable, "participants reporting consequences" was operationalized as participants positively endorsing any binge drinking consequence item on the DrInc for each type of consequence and the total score. The type of consequence variable was operationalized as each of the five consequence factors of the DrInc (physical, interpersonal, intrapersonal, impulse control, and social responsibility). The dependent variables were scores on a general measure of EI and the five factors of EI, as determined by the EQ-i.

Six separate 2 X 5 ANOVAs were proposed to be used to examine the significant differences that exist within each of the five EQ-i factors and the total EQ score for participants identified as "binge drinkers" $(N=$ 207) with and without consequences. The EQ-i scores of participants who endorsed items within each of five binge drinking consequence factors were also compared to those who did not endorse consequences in that factor. However, three of the five DrInc factors had a very low representation of participants who reported no consequences for that 
factor: physical $(N=4)$, interpersonal $(N=18)$, and impulse control $(N=$ 15); the remaining two DrInc factors were intrapersonal and social responsibility which had 63 and 61 participants indicating consequences, respectively. Only one participant was identified as a binge drinker without any consequences endorsed on the five scales of the DrInc. The low sample sizes for these factors contributed to a statistical power that was too low for analyzing the data fairly using the above approach. As a result, the relationship between the two continuous variables of general emotional intelligence, as determined by the EQ-i, and total binge drinking consequences, as determined by the DrInc, was explored for the 207 binge drinkers using the Pearson $r$ correlation analysis.

3) Are there emotional and interpersonal components of EI that predict different types of alcohol-related consequences across binge drinking college students?

The independent or predictor variables for Research Question 3 were the five "Emotional Intelligence Factors" (intrapersonal, interpersonal, stress management, adaptability, and general mood), as assessed by the EQ-i. The dependent or criterion variables were the five “Binge Drinking Consequence Factors" (physical, interpersonal, 
intrapersonal, impulse control, and social responsibility), as assessed by the DrInc.

Stepwise multiple regression analyses were used to identify the combination of EI factors that best predicted each of the five binge drinking consequence variables.

4) If significant relationships exist among EI factors and specific types of alcohol-related consequences, are there any variations across demographic variables?

The predictor variables of each significant regression were then be analyzed, by t-tests and one-way analyses of variance (ANOVAs), across each of the demographic variables to determine if differences in these predictors existed for age, gender, ethnicity, undergraduate class, SES groups, or athletic or greek membership. If the analyses of variance yielded a significant $\mathrm{F}$ value, a Tukey test for multiple comparisons was computed to determine which groups within the demographic variable differed on that emotional intelligence factor. 


\section{CHAPTER FOUR}

Results

The sample for the current study was comprised of 309 undergraduate students enrolled in introductory and advanced courses in psychology and education from two small, rural, liberal arts colleges in the northeastern United States and from one large, urban university in the Midwest. The complete demographic frequencies and percentages are provided in Table 3 (Appendix D). In terms of the differences in demographic variables, the gender of the participants was unequally divided with substantially more females $(N=198$; $64.1 \%)$ being represented than males $(N=111 ; 35.9 \%)$. The racial/ethnic demographics of the sample included a vast majority of Caucasian/White participants $(N=274 ; 88.7 \%)$, followed by participants who self-identified as being Hispanic-American or Latino/Latina $(N=8 ; 2.6 \%)$. The remaining participants made up $1.9 \%$ or less of the sample for each of the other racial/ethnic groups with five participants choosing not to answer this item. Due to the low representation from non-Caucasian/White groups, participant race/ethnicity was not used in any further statistical analyses.

The age distribution of the participants was as follows: (a) there were no participants under the age of 18, (b) participants between the ages of 18 and 20 were $47.2 \%$ of the sample $(N=146)$, (c) participants between the ages of 21 and 23 were $41.1 \%$ of the sample $(N=127)$, and $(d)$ participants age 24 and older were $11.7 \%$ of the sample $(N=36)$. Seniors $(N=111 ; 35.9 \%)$ had the highest 
number of participants for class year with sophomores $(N=73 ; 23.6 \%)$ and juniors $(N=70 ; 22.7 \%)$ at almost the same level of representation; there were 55 freshmen (first-year students) making up $17.8 \%$ of the sample. The participant sample also included 59 intercollegiate athletes (19.1\%) and 52 members of a fraternity or sorority $(16.8 \%)$.

The largest percentages of participants for socioeconomic status (SES) were primarily split between the highest, "above 100,000 " $(N=44,14.2 \%)$, and lowest choices, "below 20,000" ( $N=77 ; 24.9 \%)$; the percentages of participants in each of the other four SES categories fell between $6.5 \%$ and $8.4 \%$. It should be noted that 96 participants $(31.1 \%)$ chose not to answer this item. The reason for this bimodal distribution among SES status may reflect the questions asked of the examiner by several participants during the administration of the assessments. Some participants requested clarification as to whether to answer this item based on their own personal income and or their parents' combined income. The participants were told to use their personal income; however, other participants may have chosen to mark the item that included their parents' income without questioning the examiner. As a result, participant SES was not used in any further statistical analyses.

\section{Preliminary Analyses}

The data collected for the current study were analyzed using both descriptive and inferential statistics. The differences that exist within each of the demographic variables for EI and for binge drinking status, frequency, and 
consequences were examined by t-tests and one-way analyses of variance (ANOVAs) before the statistical analysis of the four research questions. These procedures illustrated how differences within the demographic variables of this study compare to differences observed in prior research and provided descriptive characteristics of the sample. The demographic variables included in the statistical analyses were age, class year, gender, intercollegiate athlete status, and greek system membership. Race/ethnicity and SES were eliminated as variables for the analyses due to the lack of racial diversity from non-Caucasian/White racial/ethnic groups and to the possible differences in the definition of household income for SES which may have led to the bimodal distribution.

Demographic differences in emotional intelligence. For each of the five remaining demographic variables, differences in total EI scores, as measured by the BarOn EQ-i, were assessed via t-tests and ANOVAs. A one-sample t-test was first conducted to compare the mean EQ-i score for the entire sample $(M=96.76$; $S D=12.73)$ to the mean EQ-i score for the 20 to 29 age range $(M=96.8)$ reported in the norms (Bar-On, 1997, 2002); the mean for the 16 to 19 age range was listed as 95.3. There were no reported standard deviations for either EQ-i age group, although the standard deviation is 15 for general population. At least $41.1 \%$ of the subjects from the present sample fell in the 20 to 29 age range in addition to an undetermined percentage of those 20 and 24 to 29 years of age due to age limits of the groups on the Research Questionnaire. No significant differences were observed between the EQ-i means for the present data set and 
the 20 to 29 age group, as computed by the SPSS statistical software application (release 7.0). This result suggests that the sample in the current study was consistent with the representative sample of individuals in the 20 to 29 age norm group of the EQ-i, $t(308)=.05, p>.05$.

In terms of the demographic differences on the EQ-i, Tables 4 through 8 contain the EQ-i means, standard deviations, standard error scores, and ranges for the subgroups of each demographic variable. EQ-i total scores were only found to differ on the gender demographic variable alone with males $(M=99.1 ; S D=$ 13.27) having significantly higher total EQ-i scores than females $(M=95.45 ; S D$ $=12.25), t(307)=2.43, p<.05$. As a result, separate t-tests and MANOVAs were used for each gender to answer Research Question 1. The remaining research questions were not effected by the gender differences in EQ-i scores because only binge drinkers were included in the statistical analyses for research questions 2 through 4. Furthermore, gender differences for EQ-i scores were not observed to be significant when a t-test was conducted on binge drinkers alone. Binge drinking males $(N=86, M=98.69 ; S D=12.93)$ who make up $77 \%$ of the total male sample did not have significantly different EQ-i total scores than binge drinking females $(N=121, M=95.88 ; S D=11.96)$ who make up $61 \%$ of the total female sample, $t(205)=1.61, p>.05$.

In contrast to gender, there were no significant main effects found for age, $F(2,306)=1.44, p>.05$, and class year, $F(3,305)=.85, p>.05$, on EQ-i scores. Age had the following EQ-i means and standard deviations for the four age 
groupings: (a) there were no participants under the age of 18 , (b) ages 18 to 20 ( $M$ $=96.08 ; S D=12.46),(\mathrm{c})$ ages 21 to $23(M=98.14 ; S D=12.75)$, and $(\mathrm{d})$ ages 24 and over $(M=94.7 ; S D=13.54)$. The EQ-i means and standard deviations for the four class year groups were: (a) freshmen/first-year students $(M=94.71 ; S D=$ 13.3), (b) sophomores $(M=96.62 ; S D=13.31)$, (c) juniors $(M=98.36$; $S D=$ $10.9)$, and (d) seniors $(M=96.87 ; S D=13.13)$.

The results of t-tests conducted to discern what differences exist in EQ-i scores for intercollegiate athletes and members of the greek system were also not significant. Athletes had a mean total EQ-i score of $98.97(S D=13.04)$ as compared to non-athletes whose mean EQ-i score was $96.24(S D=12.62), t(307)$ $=1.07, p>.05$. Similarly, the mean EQ-i score for members of fraternities and sororities was $99.62(S D=12.98)$ as compared to $96.25(S D=12.61)$ for nonmembers, $t(307)=1.77, p>.05$.

Demographic differences in binge drinking frequencies. In terms of the prevalence of binge drinking in the sample, 207 participants (67\%) met the criteria for binge drinking for their respective gender, as determined by Items 2 and 3 of the Research Questionnaire. The percentage (76.6\%) of male binge drinkers $(N=85)$ was significantly higher than the percentage $(61.1 \%)$ of female binge drinkers $(N=121), \chi^{2}(1, N=309)=8.62, p<.01$. Greek system members also had a significantly higher percentage of binge drinkers $(80.8 \%)$ than nonmembers $(64.2 \%), \chi^{2}(1, N=309)=5.37, p<.05$. There were no significant differences in the percentage of binge drinkers within age, $\chi^{2}(2, N=309)=5.61$, 
$p>.05$; class, $\chi^{2}(3, N=309)=2.83, p>.05$; or athlete status, $\chi^{2}(1, N=309)=$ $1.14, p>.05$

In order to explore demographic differences among the number of binge drinking episodes, Item 3 of the Research Questionnaire was used to assess the frequency of binge drinking episodes by a 6-point Likert-type scale. The question read, "FEMALES: How often do you have 4 or more drinks of alcohol on one occasion? MALES: How often do you have 5 or more drinks of alcohol on one occasion?" The possible responses were: "Never" (coded as 1), "Rarely" (coded as 2), "Monthly" (coded as 3), "Every 2 weeks" (coded as 4), "Weekly" (coded as 5), and "Daily or almost daily" (coded as 6). Tables 9 through 13 contain binge drinking frequency data from Item 3 regarding the sample sizes, means, standard deviations and standard error scores of just binge drinking participants within the subgroups of each demographic variable (age, class year, gender, athlete status, and greek system membership).

For each of the following demographic variables, there were no significant differences in binge drinking frequency rates. Participants in the 18 to 20 age group had the highest mean binge drinking frequency score $(N=99, M=4.33, S D$ $=.93)$ which was closely followed by the 21 to 23 age group $(N=90, M=4.18$, $S D=.99)$. Binge drinkers in the 24 and over age group had a lower mean frequency score $(N=18, M=3.72, S D=1.27)$ than the other two age groups, but the difference was not significant, $F(2,204)=3.0, p>.05$. The data for class year yielded a similar decline in the means of binge drinking frequencies with 
freshmen (first-year students) having the highest mean score $(N=32, M=4.38$, $S D=1.04)$, followed by sophomores $(N=51, M=4.31, S D=.99)$, juniors $(N=$ $50, M=4.22, S D=.79)$, and seniors $(N=74, M=4.08, S D=1.1)$; again, these differences among class year subgroups were not significant, $F(3,203)=1.0, p>$ .05 . Males $(N=86)$ had a mean binge drinking frequency score of $4.36(S D=.93)$ while females $(N=121)$ had a mean of $4.11(S D=1.03), t(205)=1.8, \mathrm{p}>.05$. The mean for intercollegiate athletes was $4.47(N=43, S D=.91)$ as compared to a mean of $4.15(N=164, S D=1.01)$ for non-athletes, $t(205)=1.9, \mathrm{p}>.05$. Members of the greek system $(N=42)$ had a mean frequency score of $4.26(S D=$ 99) which was almost identical to the mean of nonmembers $(N=165, M=4.2$, $S D=1), t(205)=.4, p>.05$. Although all of the above group differences were not significant in terms of the rate of binge drinking episodes, the results indicated that unique binge drinking patterns were not characteristic of any demographic subgroup.

Demographic differences in binge drinking consequences. The sample of binge drinkers (207) was used again to examine how binge drinking consequences may differ across the demographic variables. Binge drinking consequence score for each participant was determined by the total score of the DrInc. The values of the DrInc scores were the sum total of all the alcohol-related consequences that were endorsed by each participant. Tables 14 through 18 contain the means, standard deviations, standard error scores, and ranges of the total DrInc scores for the subgroups of each demographic variable. 
A one-way ANOVA exploring differences in binge drinking consequences among age groups revealed a significant main effect, $F(2,204)=6.35, p<.01$. Follow up multiple comparisons by the Tukey HSD test (results in Table 19) demonstrated significantly more binge drinking consequences reported by participants in the 24 and over age range $(M=17.67, S D=8.58)$ than participants in the 18 to 20 age range $(M=11.51, S D=6.27)$; the mean for the 21 to 23 age range was $13.81(S D=8)$. This finding may be due to the cumulative nature of the DrInc in which consequences from alcohol use were reported over a participant's lifetime. As participants age and continue to binge drink, it is likely that the number and variety of alcohol-related consequences experienced would increase over time.

Likewise, a significant main effect using a one-way ANOVA was seen for class year and binge drinking consequences, $F(3,203)=4.84, p<.01$. Follow up multiple comparisons by the Tukey HSD test (results in Table 20) revealed two significant differences in class year. Seniors $(M=15.31, S D=8.2)$ endorsed significantly more binge drinking consequences on the DrInc than freshman (firstyear students) $(M=9.91, S D=6.23)$ and sophomores $(M=11.88, S D=7.04)$; the mean for juniors was $12.88(S D=6.59)$.

Differences observed in the other demographic variables were not significant for binge drinking consequences. Males had a mean total binge drinking consequence score of $13.2(S D=8.14)$ which was slightly higher than females $(M=12.93, \mathrm{SD}=6.98)$ but not significant, $t(205)=.25, p>.05$. 
Intercollegiate athletes $(M=12.86, S D=6.85)$ reported slightly lower mean scores than non-athletes $(M=13.09, S D=7.63), t(205)=.18, p>.05$.

Additionally, members of the greek system had a mean binge drinking consequence score of $12.83(S D=8.58)$ as compared to a score of $13.1(S D=7.2)$ for nonmembers, $t(205)=.20, p>.05$.

\section{Research Question 1}

Do college binge drinkers and non-binge drinkers differ on a measure of emotional intelligence (EI)?

In order to compare general EI levels based on binge drinking status (binge drinker vs. non-binge drinker), the proposed statistical analyses were performed for each gender due to the significant difference, $t(307)=2.43, p<$ $.05)$ between males $(M=99.1 ; S D=13.27)$ and females $(M=95.45 ; S D=12.25)$ for general emotional intelligence. Therefore, a t-test was used to assess the differences in binge drinking status on the dependent variable of general emotional intelligence for each gender. Similarly, two MANOVAs (one for each gender) were conducted to explore differences that exist between binge drinkers and non-binge drinkers on the five factors of emotional intelligence: intrapersonal, interpersonal, stress management, adaptability, and general mood, as assessed by the BarOn EQ-i.

The statistical analysis revealed that there was not a significant difference between female binge drinkers $(N=77, M=94.04, S D=12.34)$ and non-binge drinkers $(N=121, M=96.36, S D=12.16)$ on a measure of general emotional 
intelligence, $t(205)=1.3, p>.05$. The results of the MANOVA conducted to determine differences between female binge drinkers and non-binge drinkers on the five factors of EI was also not significant, $F(5,192)=1.07, p>.05$.

For males, similar results were found. The difference between male binge drinkers $(N=86, M=98.69, S D=12.93)$ and non-binge drinkers $(N=25, M=$ $100.52, S D=14.55)$ was not significant for general emotional intelligence, $t(205)$ $=1.61, p>.05$. Additionally, no significant differences were observed on the MANOVA between male binge drinkers and non-binge drinkers on the five factors of EI, $F(5,105)=.63, p>.05$.

A summary of the findings for Research Question 1 demonstrates that participants who binge drink did not differ from non-binge drinkers on a measure of general emotional intelligence and the five factors of EI: intrapersonal, interpersonal, stress management, adaptability, and general mood. These results were consistent within each gender regardless of males having been shown to have significantly higher scores for general emotional intelligence scores than females.

Research Question 2

Do binge drinkers with consequences differ from binge drinkers without consequences on a measure of EI?

In order to study the effect that a reported history of any of five binge drinking consequences (physical, intrapersonal, interpersonal, impulse control, and social responsibility) may have on EI, participant consequence data were 
initially going to be compared via separate 2 X 5 (Binge Drinking Consequence Status x Type of Consequence) ANOVAs for the six dependent variables (general emotional intelligence and its five EI factors). Of the 207 participants meeting the criteria for binge drinking, only one participant did not report any alcohol-related consequences. Exceptionally low participant numbers who were without consequences were also found for three of the consequence factors: physical $(N=$ $4)$, interpersonal $(N=18)$, and impulse control $(N=15)$; intrapersonal and social responsibility consequences were not reported for 63 and 61 participants, respectively.

The low sample sizes in the consequence factors would lead to insufficient statistical power to analyze the data in a way that is representative of the population. Therefore, Research Question 2 was modified to examine the overall relationship between general emotional intelligence, as measured by the total score on EQ-i, and total binge drinking consequences, as measured by the DrInc. The correlation identified if participant EI and reported binge drinking consequences were in some way related.

A two-tailed, Pearson $r$ correlation analysis was used for the 207 binge drinking participant data which yielded a significant negative correlation $(r=-.36$, $p<.01, r^{2}=.13$ ). As participants' general emotional intelligence scores increased, the total number of alcohol-related consequences indicated on the DrInc decreased. 


\section{Research Question 3}

Are there emotional and interpersonal components of EI that predict different types of alcohol-related consequences across binge drinking college students?

The question of which EI factor or combination of factors predict each of the five binge drinking consequences was examined via five stepwise multiple regression analyses for the 207 binge drinkers. The five predictors entered into the models were the intrapersonal, interpersonal, stress management, adaptability, and general mood factors of EI. The five criterion variables were the physical, intrapersonal, interpersonal, impulse control, and social responsibility binge drinking consequence factors from the DrInc. Results from all five regression analyses revealed significant models for each criterion variable.

In terms of the physical consequence criterion, the regression analysis produced a significant finding, $\mathrm{R}-\mathrm{Square}=9.8 \%($ Table 21$), F(1,205)=22.25, p$ $<.001$ (Table 22). The component analysis demonstrated a significant contribution from the intrapersonal EI factor, $t(205)=4.72, p<.001$, (Table 23). The results indicated that the variance in physical consequences was best predicted by the intrapersonal EI measure; participants who had high intrapersonal EI scores reported few physical consequences from binge drinking.

The second regression analysis was significant for the intrapersonal consequence variable, $\mathrm{R}-\mathrm{Square}=7.1 \%($ Table 24$), F(1,205)=15.66, p<.001$ (Table 25). The component analysis demonstrated a significant contribution from the stress management EI factor, $t(205)=3.96, p<.001$, (Table 26). The results 
indicated that only one model was found that included the stress management factor as a significant predictor of intrapersonal consequences. Participants who score high on the stress management EI measure reported a low number of intrapersonal binge drinking consequences.

The interpersonal consequence variable also had a significant regression analysis, $\mathrm{R}-$ Square $=13.9 \%($ Table 27$), F(2,204)=16.44, p<.001$ (Table 28$)$. The component analysis demonstrated significant contributions from the stress management, $t(204)=4.56, p<.001$, and interpersonal, $t(204)=2.42, p<.05$, EI factors (Table 29). The results indicated that the stress management and interpersonal EI factors were the significant predictors of interpersonal consequences. Participants who scored high on the stress management and interpersonal EI factors reported a low number of interpersonal binge drinking consequences.

In terms of the impulse control criterion, the regression analysis produced a significant finding, $\mathrm{R}-$ Square $=9.8 \%($ Table 30$), F(2,204)=11.11, p<.001$ (Table 31). The component analysis demonstrated significant contributions from the stress management, $t(204)=3.4, p<.001$, and interpersonal, $t(204)=2.45, p$ $<.02$, EI factors (Table 32). The results indicated that the stress management and interpersonal EI factors were also the significant predictors of impulse control consequences. Participants who scored high on the stress management and interpersonal EI factors reported a low number of impulse control consequences with binge drinking. 
Lastly, a significant regression analysis was found for the social responsibility criterion variable, $\mathrm{R}-\mathrm{Square}=71.4 \%($ Table 33$), F(1,205)=$ 512.53, $p<.001$ (Table 34). The component analysis demonstrated a strong, significant contribution from the interpersonal EI factor, $t(205)=22.64, p<.001$ (Table 35). The results indicated that the interpersonal factor was a significant predictor of social responsibility consequences. Participants who scored high on the interpersonal EI factor reported a low number of social responsibility binge drinking consequences.

A summary of the findings for Research Question 3 shows that the variance within each of the binge drinking consequences was significantly predicted by one or more of the EI factors. The stress management, interpersonal, and intrapersonal EI factors were found to be the only significant predictors included in the above models for the binge drinking consequence factors. When the scores of these EI factors were high within their regression models, there were lower reported binge drinking consequences.

\section{Research Question 4}

If significant relationships exist among EI factors and specific types of alcoholrelated consequences, are there any variations across demographic variables?

To answer the question of how the demographic variables may be a factor on the significant EI predictors of the binge drinking consequences, t-tests and ANOVAs were conducted for the demographic variables using the significant EI predictors from Research Question 3 as dependent variables. A Tukey test for 
multiple comparisons followed all significant ANOVAs. The five significant multiple regression analyses in Research Question 3 yielded a total of three EI factors that were significant predictors of binge drinking consequences: stress management, interpersonal, and intrapersonal. It should be noted that only five (age, gender, class, athlete status, and greek membership) of the possible seven demographic variables were used in these analyses due to the lack of racial diversity in the sample ( $88.7 \%$ Caucasian/White) and to the confusion regarding the reporting of household income (self vs. parent income).

Stress management was a significant predictor of three binge drinking consequences: intrapersonal, interpersonal, and impulse control. A significant difference was demonstrated for gender in which binge drinking males $(M=$ $101.81, S D=11.4$ ) had higher scores on the stress management EI factor than binge drinking females $(M=94.84, S D=12.36), t(205)=4.13, p<.001$; this gender difference for the stress management EI factor was consistent with the literature (Bar On, 1997/2002). Binge drinking athletes $(M=101.95, S D=10.73)$ had significantly higher stress management scores than non-athletes $(M=96.63$, $S D=12.64), t(205)=2.53, p<.02$, and the higher mean scores of binge drinking, greek system members $(M=103.52, S D=11.10)$ were also significant, $t(205)=$ $3.5, p=.001$, compared to the scores of non-members $(M=96.19, S D=12.35)$. Sixteen binge drinking participants were both athletes and greek system members which represent $37 \%$ of binge drinking athletes and $38 \%$ of the binge drinking 
greek system sample. No significant findings for main effects or t-tests were observed for age, $F(2,204)=1.84, p>.05$ or class, $F(3,203)=1.73, p>.05$.

The interpersonal EI factor was also a significant predictor of three binge drinking consequence variables: interpersonal, impulse control, and social responsibility. A significant difference was demonstrated for gender in which binge drinking females $(M=103.81, S D=11.55)$ had higher scores on the interpersonal EI factor than binge drinking males $(M=96.51, S D=15.33), t(205)$ $=3.97, p<.001)$; this gender difference for the interpersonal EI factor was consistent with the literature (Bar On, 1997/2002). Additionally, binge drinking greek system members $(M=94.69, S D=15.61)$ had significantly lower interpersonal scores than non-members $(M=102.55, S D=12.7), t(205)=3.41, p$ $=.001$. No significant findings for main effects or t-tests were observed for age, $F$ $(2,204)=1.7, p>.05$, class, $F(3,203)=.62, p>.05$, or athlete status, $t(205)=$ $1.7, p>.05$

The intrapersonal EI factor was only a significant predictor of the physical binge drinking consequence variable, according to the data from Research Question 3. A significant difference was demonstrated for gender in which binge drinking females $(M=101.5, S D=14.15)$ had higher scores on the intrapersonal EI factor than binge drinking males $(M=96.85, S D=13.94), t(205)=2.35, p<$ .05. No significant main effects or t-test results were observed for age, $F(2,204)$ $=2.54, p>.05 ;$ class, $F(3,203)=1.01, p>.05 ;$ athlete status, $t(205)=.25, p>$ .05 ; or greek system membership, $t(205)=1.2, p>.05$. 
A summary of the findings for Research Question 4 demonstrates that gender had significant differences on all three significant EI predictors (stress management, interpersonal, and intrapersonal) of the five binge drinking consequence factors. However, females had higher scores than males on the interpersonal and intrapersonal EI factors, and males had higher stress management EI scores than females. Additionally, being a member of an intercollegiate athletic team or the greek system led to significantly higher stress management EI factor scores than non-members. In contrast, greek system members had significantly lower interpersonal EI factor scores than nonmembers. The age and class demographic variables did not have any significant effect on the three EI factor predictors. 


\section{CHAPTER FIVE}

\section{Discussion}

The results of the present study provide greater awareness of how the construct of emotional intelligence (EI) can be used to understand college student binge drinking and its consequences. The prevalence of binge drinking was first explored among the demographic variables (age, athlete status, class, gender, greek system membership, race/ethnicity, and SES) in relation to general emotional intelligence. Specific types of binge drinking consequences were then examined to determine if they could be predicted by the factors of EI and if demographic differences exist for significant EI predictors. The results yielded support for the findings of prior studies related to binge drinking and its consequences and provided new directions for further research.

\section{College Student Binge Drinking and Emotional Intelligence}

The prevalence of college student binge drinking found in the present sample (67\%) was substantially higher than the widely accepted figure of $45 \%$ demonstrated by the national survey conducted by Wechsler, Lee, Kuo et al. (2002). This higher percentage may be due to the disproportionate number of Caucasian/White participants and greek system members in the current sample as compared to Wechsler, Lee, Kuo et al.'s sample. Caucasian/White participants and greek system members were reported by Wechsler, Lee, Kuo et al. (2002) to have higher binge drinking percentages than the general sample; $74 \%$ of their sample was White (50.2\% of Whites were binge drinkers), and $12 \%$ were 
members of fraternities/sororities $(64.3 \%$ were binge drinkers, $75.4 \%$ when they lived in the greek house). In the present study, $88.7 \%$ of the sample was Caucasian/White (69.7\% were binge drinkers), and 16.8\% were members of the greek system (80.8\% were binge drinkers). Recent research has supported a higher percentage of greek system binge drinkers by observing that $86 \%$ of greek members met the criteria of binge drinker and that $64 \%$ fell into the category of frequent binge drinker (Caudill et al., 2006). Other possible explanations for the disparity in binge drinking prevalence rates could be the lack of similarity in the geographic locations and type of colleges between the two samples.

The data from the Research Questionnaire also demonstrated that males have a higher percentage of binge drinkers $(76.6 \%)$ than females $(61.1 \%)$. These percentages were higher than those reported by Wechsler, Lee, Kuo et al. (2002) who found $48.6 \%$ of males and $40.9 \%$ of females to be binge drinkers, but the trend was consistent with the literature which offers strong evidence that males typically drink higher quantities of alcohol than females (Humara \& Sherman, 1999; Poikolainen et al., 2001; Quigley \& Marlatt, 1996). Another prevalence rate from this study that was supported by previous research was the higher percentage of greek system members who were binge drinkers $(80.8 \%)$ than nonmembers (64.2\%) (Meilman et al., 1999; Wechsler, Lee, Kuo, et al., 2002).

The lack of significant difference in binge drinking percentages within the other demographic variables of age, class, and athlete status contradicted the findings of Leichliter et al. (1998) and Weingardt et al. (1998). However, 
Wechsler, Lee, Kuo, et al. (2002) showed that there was variability in the percentage of binge drinkers for each class year and age group over the four surveys that were conducted between 1993 and 2001. Binge drinking percentages tended to vary across the different age groups and class years without any significant trend observed in the data across the four surveys. Additionally, there were no significant differences in the frequency of binge drinking episodes found in the present study for any of the included demographic variables of binge drinkers. College binge drinkers appeared to have similar rates of binge drinking episodes regardless of the demographic groups to which they belong.

In terms of EI, there was no significant difference found between the mean total EI score of the current sample and the mean total EI score for the 20 to 29 age group reported by Bar On (1997/2002). However, males in the present study were observed to have significantly higher total EQ-i scores than females. The lower scores for females may have been a function of seven of nine of the lowest total EQ-i scores coming from female participants. No other demographic variables were shown have significant differences in total EQ-i scores.

In order to address Research Question 1, the statistical analyses performed on the data gathered from the Research Questionnaire and the BarOn EQ-i revealed that there were no significant differences between college student binge drinkers and non-binge drinkers on a measure of general emotional intelligence (total EI scores). The factors that are included in the general EI scores are intrapersonal EI, interpersonal EI, stress management, adaptability, and general 
mood. This outcome was found for both males and females when the data were analyzed separately due to the significant gender differences observed in total EI scores.

The results suggested that college students who engage in binge drinking can not be distinguished from non-binge drinkers on the basis of EI alone. The level of a college student's EI or emotional/interpersonal functioning did not seem to relate to the occurrence of binge drinking. Furthermore, binge drinking appeared to be a behavior that occurs similarly across all levels of EI and within most demographic groups of the college students sampled, excluding gender and greek system membership. However, the emotional and interpersonal components of binge drinking became increasingly important when consequences were considered.

This complex view of binge drinking is consistent with reviews of the alcohol consumption literature by Baer (2002) and Dowdall and Wechsler (2002) who concluded that increased alcohol use can be best understood by a context larger than the personality characteristics of the drinker. Multiple individual differences, such as in sociability and in the motives and intentions to drink alcohol, and the college alcohol environment seemed to be significantly involved in alcohol intake. Fondacaro and Heller (1983) added that the mental health symptoms of college students related less to alcohol consumption when other social variables regarding the individual were factored into the analyses. As important as social factors appear to be for an individual's drinking level, Baer 
(2002) also noted how the intended use of alcohol to manage affect was related to more negative consequences than the consumption of alcohol intended to enhance socialization.

Overall, the current study suggests that EI, as a general construct of an individual's ability to succeed in relationships and in coping with life stressors to create a sense of psychological well-being, does not have a significant, direct relationship to binge drinking. This finding would add credence to a proposal by Schulenberg et al. (2001) who stated that binge drinking may be considered an appropriate developmental stage for a subpopulation of college students. The results from the present study support the theory that college student binge drinking is a complex phenomenon involving multiple variables beyond the individual's psychological well-being. Furthermore, the data expand the understanding that binge drinking consequences, as opposed to the act of binge drinking, is related to the EI of a college student and that these consequences can be predicted by the factors of EI.

\section{Using EI to Understand Binge Drinking Consequences}

Although EI was not found to be related to alcohol consumption at a binge drinking level, the number of binge drinking consequences was significantly correlated with the total EI scores of participants. As EI scores increased for college students, it appeared that the number of binge drinking consequences they report decreased. It should be noted that the original intent of Research Question 2 was to explore if binge drinkers with and without consequences differed on a 
measure of EI and its factors. However, the extremely low sample sizes of binge drinkers without consequences for most of the binge drinking consequence types prohibited the proposed statistical analyses for the research question. Further research with larger sample sizes would help clarify the differences that may exist between binge drinkers with and without consequences.

The finding of an inverse relationship between EI and binge drinking consequences suggested that college students who experience alcohol-related consequences may have emotional and interpersonal deficits that could be related to these problems. This result is consistent with research that similarly found low emotional intelligence scores and lower quality of life satisfaction ratings to predict alcohol problems (Murphy et al., 2006; Riley \& Schutte, 2003). Several studies have also demonstrated that depression, anxiety, loneliness, and tension in college students have been related to problems associated with drinking (Bonin et al., 2000; Camatta \& Nagoshi, 1995; Carey \& Correia, 1997). Thus, the emotional and interpersonal struggles of college students may have become more evident with excessive alcohol consumption. As greater focus was placed on studying the types of binge drinking consequences, it was shown that specific deficits in EI correspond to different types of consequences.

In a closer examination of the types of consequences due to excessive alcohol consumption, the intrapersonal EI scores from binge drinking college students have been observed to significantly predict the variance in the total number of physical consequences. The components of the intrapersonal EI score 
on the EQ-i included self-regard, emotional self-awareness, assertiveness, independence, and self-actualization. These components combined represent the ability of an individual to recognize and express one's inner feelings and thoughts, to acknowledge oneself as basically good, and to pursue personally meaningful activities in a manner respectful to others and free from emotional dependency.

Lower intrapersonal EI scores were observed to be indicative of higher reports of physical consequences. Participants who have deficits in intrapersonal EI reported a greater number of physical consequences from binge drinking. Additionally, follow-up statistical analyses revealed that male binge drinkers had significantly lower intrapersonal EI scores than female binge drinkers, and thus may have been more prone to physical consequences. An increase in negative alcohol-related consequences has been previously observed in male subjects with alexithymia who have difficulty labeling and communicating affect (Kaulhanen et al., 1992). Other research has corroborated the findings from the present study by showing that alcohol-related consequences were connected to the intentional use of alcohol to manage negative affect, although these results were found separately for both males and females (Baer, 2002; Glynn et al., 1983).

The need to express and address negative emotional experiences through alcohol use may suggest deficits in emotional self-awareness and overall intrapersonal EI that could lead to physical problems. Several researchers have pointed out that even without alcohol use, the physiological effect of higher blood 
pressure was associated with a decrease in the self-awareness and expression of anger, helplessness, and hopelessness (Goldstein, Edelberg, Meier, \& Davis, 1988; Lynch, Lynch, \& Friedman, 1992). Therefore, individuals who do not have the ability to express their negative affect in an effective manner may possibly internalize their emotions to the point of experiencing physical reactions and problems when binge drinking. It is important to note that physical consequences were the only type of binge drinking consequences not predicted by either the interpersonal or stress management components of EI or a combination of both.

In terms of predicting intrapersonal binge drinking consequences, a surprising result was exhibited in which intrapersonal EI was not shown to be a significant predictor in the regression model. Stress management EI was the only predictor to explain the variance in reported intrapersonal consequences from binge drinking. The EI components of stress tolerance and impulse control made up the stress management EI factor. These EI components represented the ability of an individual to cope with stressful situations, to not become overwhelmed, and to reflect on or refrain from negative actions. Differences within the demographic variables of gender, athlete status, and greek system membership were also found to be significant in the follow-up analyses. Males, athletes, and greek system members had the highest stress management scores.

Higher stress management scores reflected lower reports of intrapersonal binge drinking consequences. These results were consistent with a study that found the sole use of avoidant stress management strategies was a significant 
predictor of alcohol-related consequences for female college students (Simpson \& Arroyo, 1998). Likewise, female college students who drank to cope with the experience of elevated sadness predicted increases in alcohol consumption and related consequences as compared to males (Hussong, 2007). In general, the experience of stress and having ineffective coping strategies have been associated with excessive alcohol consumption and related problems (Cooper et al., 1992; Ichiyama \& Kruse, 1998; O’Hare \& Sherrer, 2006). Furthermore, the use of alcohol to manage stress and the belief that alcohol will be effective in managing stress have not been shown to be helpful when attempting to reduce stress and tension, but this outcome is dependent on individual differences (Corcoran \& Parker, 1991).

The inability of alcohol to be a sufficient stress management tool may have contributed to alcohol-related consequences, such as intrapersonal consequences, for some college students who have deficits in stress management EI. An individual's sense of personal growth and capacity to manage distressing affect/mood states could have been negatively affected by binge drinking when there was little or no other means to alleviate stress but alcohol.

Interpersonal EI scores were demonstrated in the present study to be a significant predictor of the variance in social responsibility consequences from binge drinking. The EI components of empathy, social responsibility, and interpersonal relationship made up the interpersonal factor. These components represented the ability of an individual to understand the feelings of others, to be a 
cooperative and contributing social member, and to develop and maintain mutually intimate relationships. Differences in gender were again seen in the follow-up analyses with binge drinking females having significantly higher interpersonal EI scores than males; the inverse was found for the intrapersonal EI factor. Similarly, binge drinking greek system members had significantly lower interpersonal EI scores than nonmembers which also was reverse for the intrapersonal EI factor; it should be noted that only one greek system member was female which could be a reason for the significant difference observed.

Higher interpersonal EI scores were indicative of lower reports of social responsibility consequences due to binge drinking. The importance of the social aspects of binge drinking has been heavily studied in the alcohol research literature (Nezlak et al, 1994). Moreover, alcohol's role in facilitating social interaction has been viewed as having both positive and negative characteristics (Haemmerlie et al., 1999; Maggs, 1997). Prior research has provided evidence that males tend to seek alcohol consumption for the purposes of social enhancement (Murphy et al., 2006). College females who binge drink report higher levels of intimacy than non-binge drinkers, but the opposite was shown for males (Nezlak et al, 1994). Additionally, males have been shown to have a stronger tendency than females to drink more when males have a high level of social anxiety and perceive high drinking norms on campus (Neighbors, Fossos, Woods, Fabiano, Sledge, \& Frost, 2007). 
These findings along with the current research suggests that although college students (especially females) binge drink, those who had high interpersonal abilities may have continued to engage in healthy roles in their social groups due to being less vulnerable to the negative interpersonal consequences of excessive alcohol use. In contrast, college binge drinkers (especially males) may have sought alcohol as a means to improve socialization because of low interpersonal EI. This means of socialization for students with low interpersonal EI could have led to the experience of an increased level of poor judgment due to impairing effects of alcohol when following through with social responsibilities (Glynn et al., 1983).

The combined effect of the stress management and interpersonal EI predictors were significant in explaining the variance of both interpersonal and impulse control binge drinking consequences. Interestingly, the gender differences noted above for the stress management and interpersonal EI factors appeared to have been in contrast with each other. Males had higher stress management EI scores than females, and females had higher interpersonal EI scores than males. This difference in gender effects supported the finding that the stress management and interpersonal EI factors may have uniquely explained the variance in both interpersonal and impulse control consequences.

College binge drinkers with low stress management and interpersonal EI scores were more likely to have high reports of interpersonal and impulse control consequences. These results were consistent with numerous studies that attempt to 
discern the contributing factors of college student binge drinking. The inability to effectively manage emotions and impulses and to maintain self-control has been strongly linked to binge drinking (Beck et al., 1995; Quigley \& Marlatt, 1996).

Consequences from excessive alcohol consumption have also been shown to be more present in those who use alcohol primarily for affect management and then in those who drink to improve socialization (Baer, 2002; Brown, 1985; Glynn et al., 1983). However, inconsistencies have been revealed in binge drinking research studies that explore the use of alcohol to manage negative affect. This observation led to the proposal that greater emphasis should be placed on the research of individual differences and motives for binge drinking (Baer, 2002). The approach to understanding binge drinking consequences in the present study provided more clarity to the emotional and interpersonal factors involved in binge drinking and specific types of alcohol-related consequences. The current results demonstrated that binge drinking itself may not have been indicative of low overall EI but deficits in the intrapersonal, interpersonal, and stress management factors of EI may have contributed to specific types of binge drinking consequences for college students. The disinhibiting effects of alcohol may have exacerbated these EI deficits to a level in which higher numbers of related binge drinking consequences were experienced.

\section{Conclusions/Implications}

The overall results of this study suggest that the consequences occurring from college student binge drinking are multidimensional and should be studied 
using methods that distinguish the different types of consequences from each other and from the act of binge drinking. This finding supported the binge drinking literature that highlights the need to study consequences in terms of the multiple principle components or types of consequences that can be exhibited (Miller et al., 1995; O’Neill et al., 2001; Vik et al., 2000). The unique nature of binge drinking consequences is only beginning to be understood. However, the results of the present research provided evidence for the value of using a categorical framework of consequences. Such a framework could add consistency and clarity to further research on the topic or offer guidance to alcohol prevention programs to reduce the prevalence and severity of binge drinking consequences. Additionally, the construct of EI was shown to be a viable tool for researching binge drinking and its consequences due to several significant findings that yield new information about the nature of alcohol-related consequences. An important outcome of examining EI in relation to binge drinking is that alcohol consumption alone wasn't found to be the sole contributor to alcohol-related consequences, a finding consistent with similar research (Graczyk et al., 2001). College students who binge drink were observed to have increased reports of specific types of consequences with decreases in corresponding EI levels. Furthermore, the EI scores of participants who binge drink were not significantly different from non-binge drinkers, but alcohol-related consequences were shown to decrease as total EI levels became higher. 
A closer analysis of the EI deficits revealed that decreases in the stress management, interpersonal, and intrapersonal EI factors predicted increases in the five binge-drinking consequence types. Although deficits in the specific components and factors of EI have rarely been explored due to the newness of the construct, a multitude of research studies and theorists have looked at the problems associated with the use of alcohol to alleviate stress, facilitate socialization, relieve tension, and enhance mood (Bandura, 1969; Camatta \& Nagoshi, 1995; Giancola, 2002; Ichiyama \& Kruse, 1998; Schulenberg, Wadsworth, et al., 1996; Simpson \& Arroyo, 1998).

The connections among stress management, interpersonal factors, and alcohol problems have been studied extensively with some inconsistencies observed related to gender, individual differences, the importance of the stressor to the individual, and the positive expectation of alcohol to reduce negative affect (Baer, 2002; Krause, 1995). The present study may have provided a step in identifying the reason for some of the inconsistencies by demonstrating how deficits in the stress management and interpersonal functioning of an individual was associated with specific types of binge drinking consequences but not others. Additionally, analyses of both the stress management and interpersonal EI factors revealed unique significant differences between males and females that could affect the outcome of future research.

According to the present data, deficits in the stress management and interpersonal EI factors contributed to an increase in interpersonal and impulse 
control binge drinking consequences. The stress management EI factor was also inversely related to intrapersonal consequences, and the interpersonal EI factor was inversely related to social responsibility consequences. These findings suggested that when college students have a general difficulty coping with stress and interacting in meaningful relationships, binge drinking may have had a negative effect that substantially amplified an individual's inability to cope. It may have been an overwhelming challenge for these individuals to binge drink and manage stressors, affect, responsibilities, and interpersonal relationships with good judgment and patience. College students, especially men, have been found to have increased alcohol problems when using alcohol to manage negative affect and as an aid in socialization (Baer, 2002; Glynn et al., 1983). Males with dispositional aggression were also more likely to engage in aggressive behavior following alcohol intoxication than men with lower dispositional aggression (Bailey \& Taylor, 1991). These effects may have been particularly problematic because if alcohol tends to increase feelings of tension for meaningful stressors (Krause, 1995), binge drinkers may have been tempted to increase their alcohol intake in order to manage the heightened negative feelings.

Physical binge drinking consequences appeared to have a unique EI deficit source, the intrapersonal EI factor. Difficulties labeling the experience of affect, expressing oneself, and maintaining a positive self-regard seemed to have become internalized for binge drinkers with intrapersonal deficits which ultimately led to physical consequences when drinking. Other studies, not involving alcohol use, 
have supported this observation by reporting that physical illness was more common among individuals who are alexithymic and have difficulty putting their emotional experiences into words (Cox, Kuch, Parker, Shulman, \& Evans, 1994; Lumley, Tomakowsky, \& Torosian, 1997; Wise, Jani, Kass, Sonnenschein, \& Mann, 1988). Further research is recommended to better understand the connection between physical binge drinking consequences and low intrapersonal EI. Questions should continue to be explored such as if improved emotional labeling and a more positive view of oneself would lead to less physical consequences.

This study's outcomes also have relevance for alcohol prevention programs on college campuses. Prevention curricula that aim to enhance a student's self-regard, emotional labeling, stress management abilities, and interpersonal skills may produce less binge drinking consequences. Research studies in effective prevention programs have been discovering the need for a multimodal approach that includes emphasis on the emotional and interpersonal well-being of students in addition to social norms modification, psychoeducation and disciplinary action (Graczyk et al., 2001; Hawkins \& Catalano, 1992;

Wechsler, Lee, Kuo et al, 2002). If programs address alcohol problems from a variety of directions that enhance the emotional and interpersonal development of students, efforts to reduce binge drinking consequences could be reduced without the extensive resources put into decreasing consumption (Miller et al., 1995). Based on the gender differences in the results of the present study, individualized 
prevention programs that target improved stress management EI for females and interpersonal and intrapersonal EI for males are recommended for further research support. One community substance abuse prevention program has already shown positive outcomes when instructing females in life skills and males in interpersonal skills (Smith, 2002).

In summary, alcohol by its physical nature causes changes in mood and consciousness which can have a significant impact on a college student's judgment to engage in appropriate or inappropriate behaviors (Lang et al., 1999; Lazarus, 1991). This study supports and expands on prior research demonstrating that increases in different types of negative binge drinking consequences may have resulted from a combination of the disinhibiting effects of alcohol and deficits in EI (Turrisi et al., 2000). However, the act of engaging in binge drinking does not in itself seem to indicate low EI (Schulenberg et al, 2001).

\section{Limitations of the Study}

Prior to interpretation of the data, consideration should be given to the limitations of the current study in the areas of sampling, measurement, and methodology. A potential limitation was the convenience sample used to generalize the results to the population it was intending to represent, college student binge drinkers and non-binge drinkers. The representativeness of the sample may have been affected by the use of only psychology and education students in three Eastern and Midwestern colleges and universities. Although an attempt was made to increase the racial diversity of the study by soliciting 
participants from an urban university, the sample was overwhelmingly made up of Caucasian/White students. A larger sample number would also have been beneficial to increase the size of participants who fell into the subgroups of binge drinkers without consequences, athletes, and greek system members (especially sorority members). Additionally, confusion regarding the SES item (family vs. personal income) on the Research Questionnaire led to this demographic variable being removed from the data analysis. It should be noted that seven of the nine lowest EQ-i total scores were from female participants. These data may have contributed to lower mean EI scores for females being included the statistical analyses than the actual mean of the population they represent.

The measures used in the present study also posed some possible limitations. All of the assessments used to collect data on demographic characteristics, alcohol consumption rates, binge drinking consequences, and EI were self-report measures that have been rarely used in empirical research. Although the reliability and validity of self-reports regarding alcohol use have been supported by the literature, the scores were still considered subject to response distortion and missing answers (Wechsler, Lee, Kuo et al., 2002). In particular, the topics of EI, alcohol use, and binge drinking consequences are sensitive and personal areas for many college students which may have led to participants responding to items in sociably desirable ways. This could have caused some consumption rate and binge drinking consequence items to have been underreported. Another limitation was the lack of standardization of the 
Research Questionnaire that was developed specifically for this study; although, it was based on several items from the AUDIT, considered a valid and reliable measure of alcohol use.

In terms of potential limitations associated with the methodology of the study, Dowdall and Wechsler (2002) expressed caution with conducting binge drinking research that occurs at a single point in time for college students. The authors encouraged the use of longitudinal surveys to capture changes in students over time that may affect the interpretation of the results. Due to the lack of binge drinkers without consequences, a correlational design replaced the proposed research question (2) in order to explore the relationship between binge drinking consequences and total EI scores. The significant relationship that was found between the two variables and for the regression analyses should not be interpreted with implications of causality. Furthermore, data from 15 participants were eliminated from the statistical analyses due to incomplete or invalid assessments.

\section{Recommendations for Further Research}

The results of the present study provide support for many of the findings in the alcohol and binge drinking research literature but also offers new observations regarding the nature of binge drinking consequences. Replication of the study with a larger, more heterogeneous sample is recommended to corroborate the results and strengthen the generalizability of the findings to the represented population, especially those from diverse racial and SES groups. 
Questionnaires that assess SES for college students should provide clear guidance as to the source of household income (family vs. individual) due to the division within college students who either financially support themselves or receive support from another source, such as their family.

The methodology used to examine the research questions could be modified to a longitudinal study in which college students are assessed for EI and binge drinking consequences for each year of their college career. This approach would allow any trends or inconsistencies in the data to be revealed and to determine if any changes in EI for individual students correspond to changes in reported consequences. Any future study of EI and binge drinking should also take into account first, the need to further validate the assessment instruments used in empirical research with college students and secondly, the effect of gender differences on the results.

In terms of additional research that could expand on the interpretation of the data, a more detailed understanding of the nature of the assessed EI deficits may be beneficial when studying binge drinking consequences. EI measures may be revealing EI deficits that represent a lack of knowledge about a particular area (i.e., stress management skills) or that reflect a psychological barrier to using the skills already learned (i.e., an anxiety disorder). Differentiating between a lack of emotional and interpersonal abilities and potential mental illness would be a critical distinction that may affect the interpretation of the results. Some college students who binge drink with related consequences may require counseling and 
psychological treatment while others may benefit from targeted psychoeducation programs based on the type of consequence reported.

In general, additional research on newly-developed or modified alcohol prevention programs is needed to examine if there are valued outcomes to incorporating EI factors in their approach to reducing consequences. One possible approach may be targeting the improvement of the emotional and interpersonal development of an individual based on their type of binge drinking consequences. Identified students with consequences could be required to attend a prevention program that encourages the development of positive emotional and interpersonal skills reflective of their EI deficits. Prevention programs that designed to assist students already identified as having specific types of consequences may also be compared to programs that are mandatory for all students. Curricula offered to the entire student body that incorporated the intrapersonal, interpersonal, and stress management EI factors could be studied in relation to students' reported consequences over the course of their college careers.

Finally, binge drinking college students with consequences who have been able to reduce the number and variety of consequences they experience could be studied qualitatively to better understand the factors that may have been a positive influence on these changes. 


\section{References}

Abrams, D.B., \& Niaura, R.S. (1987). Social learning theory. In H.T. Blane \& K.E. Leonard (Eds.), Psychological theories of drinking and alcoholism (pp. 131-178 ) New York: Guilford Press.

Ackerlind, I., \& Hornquist, J.O. (1992). Loneliness and alcohol abuse: A review of evidences of an interplay. Social Science and Medicine, 4, 405-414.

Altura, B.M., Gebrewold, A., Zheng, A., Altura, B.T., \& Gupta, R.K. (1998). Magnesium deficiency exacerbates brain injury and stroke mortality induced by alcohol: A-sup-3-sup1P-NMR in vivo study. Alcohol, 15, 181-183.

American Psychiatric Association. (2000). Diagnostic and statistical manual of mental disorders (4th ed., text rev.). Washington, D. C.: Author.

Applebaum, S.A. (1973). Psychological-mindedness: Word, concept, and essence. International Journal of Psychoanalysis, 54, 35-45.

Aseltine Jr., R.H., \& Gore, S.L. (2000). The variable effects of stress on alcohol use from adolescence to early adulthood. Substance Use and Misuse, 35(5), 643-668.

Babor, T.F., de la Fuente, J.R., Saunders, J., \& Grant, M. (1992). AUDIT: The Alcohol Use Disorders Identification Test: Guidelines for use in primary health care. Geneva, Switzerland: World Health Organization. 
Babor, T.F., Higgins-Biddle, J.C., Saunders, J.B., \& Monteiro, M.G. (2001). The Alcohol Use Disorders Identification Test (AUDIT): Guidelines for use in primary care $\left(2^{\text {nd }}\right.$ ed.). Geneva, Switzerland: World Health Organization.

Baer, J.S. (2002). Student factors: Understanding individual variation in college drinking. Journal of Studies on Alcohol, 63(2), 40-54.

Baer, J.S., Stacy, A., \& Larimer, M. (1991). Biases in the perception of drinking norms among college students. Journal of Studies on Alcohol, 52, 580-586.

Bailey, D.S., \& Taylor, S.P. (1991). Effects of alcohol and aggressive disposition on human physical aggression. Journal of Research in Personality, 25, 334-342.

Bagby, M., \& Taylor, G.J. (1997). Affect dysregulation and alexithymia. In G. J. Taylor, Bagby, R. M., \& Parker, J. D. A. (Eds.), Disorders of affect regulation: Alexithymia in medical and psychiatric illness (pp. 26-45). New York: Cambridge University Press.

Bagby, M., Parker, J.D.A., \& Taylor, G.J. (1994). The Twenty-Item Toronto Alexithymia Scale: I, Item selection and cross-validation of the factor structure. Journal of Psychosomatic Research, 38, 23-32.

Bandura, A. (1969). Principles of behavior modification. New York: Holt, Rinehart, \& Winston.

Bar-On, R. (1997/2002). The Emotional Quotient Inventory (EQ-i): Technical manual. Toronto, Canada: Multi-Health Systems, Inc. 
Bar-On, R., \& Parker, J.D.A. (2000). Introduction. In R. Bar-On \& J. D. A. Parker (Eds.), The Handbook of Emotional Intelligence (pp. xi-xv). San Francisco: Jossey-Bass.

Beck, K.H., Thombs, D.L., Mahoney, C.A., \& Fingar, K.M. (1995). Social context and sensation seeking: Gender differences in college student drinking motivations. International Journal of the Addictions, 30, 1101-1115.

Beck, K.H., Thombs, D.L., \& Summons, T.G. (1993). The social context of drinking scales: Construct validation and relationship to indicants of abuse in an adolescent population. Journal of Addictive Behaviors, 21, 633-644.

Beck, K. H., \& Treiman, K. A. (1996). The relationship of social context of drinking, perceived social norms, and parental influence to various drinking patterns of adolescents. Addictive Behaviors, 21(5), 633-644.

Bell, R., Wechsler, H., \& Johnston, L.D. (1997). Correlates of college student marijuana use: Results of a U.S. national survey. Addiction, 92, 571-581.

Bennett, M.E., Miller, J.H., \& Woodall, W.G. (1999). Drinking, binge drinking and other drug use among southwestern undergraduates: Three-year trends. American Journal of Drug and Alcohol Use, 25(2), 331-350.

Berenbaum, H., \& Irvin, S. (1996). Alexithymia, anger, and interpersonal behavior. Psychotherapy and Psychosomatics, 65(4), 203-208.

Berkowitz, A.D., \& Perkins, H.W. (1987). Recent research on gender differences in collegiate alcohol use. Journal of American College Health, 36, 123-129. 
Bishop, J.B. (2000). An environmental approach to combat binge drinking on college campuses. Journal of College Student Psychotherapy, 15(1), 15-30.

Blazina, C., \& Watkins, C.E. (1996). Masculine gender role conflict: Effects on college men's psychological well-being, chemical substance usage, and attitudes toward help-seeking. Journal of Counseling Psychology, 43(4), 461-465.

Block, J. (1995). On the relation between IQ, impulsivity and delinquency: Remarks on the Lynam, Moffitt, and Stouthamer-Loeber interpretation. Journal of Abnormal Psychology, 104, 395-398.

Bohn, M.J., Babor, T.F., \& Kranzler, H.R. (1995). The Alcohol Use Disorders Identification Test (AUDIT): Validation of a screening instrument for use in medical settings. Journal of Studies on Alcohol, 56, 423-432.

Bonin, M. F., McCreary, D. R., \& Sadava, S. W. (2000). Problem drinking behavior in two community-based samples of adults: Influence of gender, coping, loneliness, and depression. Psychology of Addictive Behaviors, 14(2), 151-161.

Borges, N.J., \& Hansen, S.L. (1993). Correlation between college students' driving offenses and their risks for alcohol problems. Journal of American College Health, 42, 79-81.

Boyatzis, R.E., Goleman, D., \& Hay/McBer. (1999). Emotional competence inventory. Boston: HayGroup. 
Bradstock, M.K., Forman, M.R., Binkin, N.J., Gentry, E.M., Hogelin, G.C., Williamson, D.F., et al. (1988). Alcohol use and health behavior lifestyles among U.S. women: The behavioral risk factor surveys. Addictive Behaviors, 13(1), 61-71.

Brennan, P.L., Moos, R.H., \& Mertens, J.R. (1994). Personal and environmental risk factors as predictors of alcohol use, depression, and treatment seeking: A longitudinal analysis of late-life problem drinkers. Journal of Substance Abuse, 6, 191-208.

Brennan, A.F., Walfish, S., \& AuBuchon, P. (1986). Alcohol use and abuse in college students. I. A review of individual and personality correlates. International Journal of the Addictions, 21, 449-474.

Brondolo, E. (1996). Correlates of risk for conflict among New York City traffic agents. In G. R. VandenBos \& E. Q. Bulatao (Eds.), Violence on the job: Identifying risks and developing solutions. Washington, DC: American Psychological Association.

Brown, S.A. (1985). Expectancies versus background in the prediction of college drinking patterns. Journal of Consulting and Clinical Psychology, 53, 123-130.

Browning, M.D., Hoffer, B.J., \& Dunwiddie, T.V. (1992). Alcohol, memory, and molecules. Alcohol Research and Health World, 16, 280-284. 
Bushman, B.J., \& Cooper, H.M. (1990). Effects of alcohol on human aggression: An integrative research review. Psychology Bulletin, 107, 341-354.

Butterfield, P.S., \& LeClair, S. (1988). Cognitive characteristics of bulimic and drug abusing women. Addictive Behaviors, 13, 131-138.

Caetano, R., \& Herd, D. (1984). Black drinking practices in Northern California. American Journal of Drug and Alcohol Abuse, 10(4), 571-587.

Cahalan, D., Cisin, I.H., \& Crossley, H.M. (1969). American drinking practices: A national study of drinking behavior and attitudes. New Brunswick, NJ: Rutgers Center of Alcohol Studies Monograph No. 6.

Caldwell, L.L., Kivel, B.D., Smith, E.A., \& Hayes, D. (1998). The leisure context of adolescents who are lesbian, gay male, bisexual and questioning their sexual identities: An exploratory study. Journal of Leisure Research, $30(3), 341-355$.

Caldwell, P.E. (2002). Drinking levels, related problems and readiness to change in a college sample. Alcoholism Treatment Quarterly, 20(2), 1-15.

Camatta, C.D., \& Nagoshi, C.T. (1995). Stress, depression, irrational beliefs, and alcohol use and problems in a college student sample. Alcoholism: Clinical and Experimental Research, 19, 142-146.

Cappell, H., \& Greeley, J. (1987). Alcohol and tension reduction: An update on research and theory. In H. T. Blane, \& K. E. Leonard (Eds.), 
Psychological theories of drinking and alcoholism (pp.15-54). New York: Guilford press.

Capraro, R.L. (2000). Why college men drink: Alcohol, adventure, and the paradox of masculinity. Journal of American College Health, 48, 307-315.

Carey, K.B., \& Correia, C.J. (1997). Drinking motives predict alcohol-related problems in college students. Journal of Studies on Alcohol, 58, 100-105.

Carey, K.B., Henson, J.M., Carey, M.P., \& Maisto, S.A. (2007). Which heavy drinking college students benefit from a brief motivational intervention? Journal of Consulting and Clinical Psychology, 75(4), 663-669.

Carpenter, K.M., \& Hasin, D.S. (1998). Reasons for drinking alcohol: relationships with $D S M-I V$ alcohol diagnoses and alcohol consumption in a community sample. Psychology of Addictive Behaviors, 12(3), 168-184.

Cattell, R.B., Eber, H.W., \& Tatsouka, M. (1970). Handbook for the Sixteen Personality Factor Questionnaire (16PF). Champaign, IL: Institute for Personality and Ability Testing.

Caudill, B.D., Crosse, S.B., Campbell, B., Howard, J., Luckey, B., \& Blane, H.T. (2006). High-risk drinking among college fraternity members: A national perspective. Journal of American College Health, 55(3), 141-155.

Chan, A.W.K, Pristach, E.A., \& Welte, J. (1994). Detection by the CAGE of alcoholism or heavy drinking in primary care outpatients and the general population. Journal of Substance Abuse, 6(2), 123-135. 
Chick, J., Ritson, B., Connaughton, J., Stewart, A., \& Chick, J. (1988). Advice versus extended treatment for alcoholism: a controlled study. British Journal of Addiction, 83, 159-170.

Ciarrochi, J.V., Chan, A.Y.C., \& Caputi, P. (2000). A critical evaluation of the emotional intelligence construct. Personality and Individual Differences, 28(3), 539-561.

Clark, W.A. (1998). Alcohol consumption in university students: The role of reasons for drinking, coping strategies, expectancies, and personality traits. Addictive Behaviors, 23, 317-378.

Claussen, B., \& Aasland, O.G. (1993). The Alcohol Use Disorders Identification Test (AUDIT) in a routine health examination of long-term unemployed. Addiction, 88, 363-368.

Cobb, C.R., \& Mayer, J.D. (2000). Emotional intelligence: What the research says. Educational Leadership, 58, 14-19.

Collins, R.L., Parks, G.A., \& Marlatt, G.A. (1985). Social determinants of alcohol consumption: The effects of social interaction and model status on the self-administration of alcohol. Journal of Consulting and Clinical Psychology, 53, 189-200.

Conigrave, K.M., Saunders, J.B., \& Reznik, R.B. (1995). Predictive capacity of the AUDIT questionnaire for alcohol-related harm. Addiction, 90, 14791485. 
Cooper, R.K. (1996/1997). EQ map. San Francisco: AIT and Essi Systems.

Cooper, M.L., Frone, M.R., Russell, M., \& Mudar, P. (1995). Drinking to regulate positive and negative moods: A motivational model of alcohol use. Journal of Personality and Social Psychology, 69, 990-1005.

Cooper, M.L., Russell, M., \& George, W.H. (1988). Coping expectancies and alcohol abuse: A test of social learning formulations. Journal of Abnormal Psychology, 97, 218-230.

Cooper, M.L., Russell, M., Skinner, J.B., Frone, M.R., \& Mudar, P. (1992). Stress and alcohol use: Moderating effects of gender, coping, and alcohol expectancies. Journal of Abnormal Psychology, 101(1), 139-152.

Corcoran, K.J., \& Parker, P.S. (1991). Alcohol expectancy questionnaire tension reduction scale as a predictor of alcohol consumption in a stressful situation. Addictive Behaviors, 16, 129-137.

Cox, W.M., \& Klinger, E. (1990). Incentive motivation, affective change, and alcohol use: A model (pp. 291-314). In W. M. Cox (Ed.), Why people drink: parameters of alcohol as reinforcer. New York: Gardner press.

Cox, B.J., Kuch, K., Parker, J.D.A, Shulman, I.D., \& Evans, R.J. (1994). Alexithymia in somatoform disorder patients with chronic pain. Journal of Psychosomatic Research, 38(6), 523-527.

Crawford, A., Plant, M.A., Kreitman, N., \& Latcham, R.W. (1987). Unemployment and drinking behaviour: Some data from a general 
population survey of alcohol use. British Journal of Addiction, 82(9), $1007-1016$.

Cronin, C. (1997). Reasons for drinking versus outcome expectancies in the prediction of college student drinking. Substance use \& misuse, 32(10), $1287-1311$

Data points: Alcohol and traffic deaths. CNN Interactive. (1997, September 7). Retrieved July 18, 2002, from http://www.cnn.com/HEALTH/9709/01/nfm.drunk.driving/data.html

Dawda, D., \& Hart, S. D. (2000). Assessing EI: Reliability and validity of the Bar-On Emotional Quotient Inventory(EQ-i). Journal of Personality and Individual Differences, 28, 797-812.

Debord, K.A., Wood, P.K., Sher, K.J., \& Good, G.E. (1998). The relevance of sexual orientation to substance abuse and psychological distress among college students. Journal of College Student Development, 39, 157-168.

Derksen, J., Kramer, I., \& Katzko, M. (2002). Does a self-report measure for emotional intelligence assess something different than general intelligence? Personality and Individual Differences, 32, 37-48.

Dowdall, G.W., \& Wechsler, H. (2002). Studying college alcohol use: widening the lens, sharpening the focus. (Panel 1: The contexts and consequences of college drinking.) Journal of Studies on Alcohol, 63(2), 14-22. 
Durkin, K.F, Wolfe, T.W., \& Clark, G. (1999). Social bond theory and binge drinking among college students: a multivariate analysis. College Student Journal, 33(3), 450-462.

Ellis, D.A., Zucker, R.A., \& Fitzgerald, H.E. (1997). The role of family influences in development and risk. Alcohol Health and Research World, 21(3), 218226.

Emmons, K.M., Wechsler, H., Dowdall, G., \& Abraham, M. (1998). Predictors of smoking among U.S. college students. American Journal of Public Health, 88(1), 104-107.

Engs, R.C., \& Hanson, D.J. (1981). The Student Alcohol Questionnaire and Alcohol Attitude Questionnaire. Bloomington, IN and Potsdam, NY: Indiana University and State University of New York.

Engs, R.C., Diebold, B.A., \& Hanson, D.J. (1994). The drinking patterns and problems of a national sample of college students, 1994. Journal of Alcohol and Drug Education, 41, 13-33.

Fillmore, M.T. (1988). Alcohol use across the life course. Toronto, Ontario: Addiction Research Foundation.

Fisher, B.S., Sloan, J.J., Cullen, F.T., \& Lu, C. (1998). Crime in the ivory tower: The level and sources of student victimization. Criminology, 36, 671-710. 
Fleming, M.F., Barry, K.L., \& MacDonald, R. (1991). The Alcohol Use Disorders Identification Test (AUDIT) in a college sample. International Journal of Addiction, 26(11), 1173-1185.

Fondacaro, M.R., \& Heller, K. (1983). Social support factors and drinking among college student males. Journal of Youth and Adolescence, 12(4), 285-299.

Forgas, J.P. (2001). Affective intelligence: The role of affect in social thinking and behavior. In J. Ciarrochi, J. P. Forgas, \& J. D. Mayer (Eds.), Emotional intelligence in everyday life: A scientific inquiry (pp. 46-63). Philadelphia: Psychology Press.

Forney, M.A., Forney, P.D., \& Ripley, W.K. (1991). Alcohol use among Black adolescents: Parental and peer influences. Journal of Alcohol and Drug Education, 36(3), 36-46.

Fukunishi, I. (1994). Social desirability and alexithymia. Psychological Reports, $75(2), 835-838$.

Fukunishi, I. (1996). College maladjustment may be related to alexithymia constructs with weakness of ego strength. Psychological Reports, 78(3, Pt. 1), $735-738$.

Fukunishi, I., \& Rahe, R. H. (1995). Alexithymia and coping with stress in healthy persons: Alexithymia as a personality trait is associated with low social support and poor responses to stress. Psychological Reports, 76(3 Pt 2), 1299-1304. 
Gardner, H. (1983). Frames of mind. New York: Basic Books.

Gardner, H. (1999, February). Who owns intelligence? Atlantic Monthly, 283.

Giancola, P.R. (2000). Executive functioning: A conceptual framework for alcohol-related aggression. Experimental Clinical Psychopharmacology, $8,576-597$.

Giancola, P.R. (2002). Alcohol-related aggression during the college years: Theories, risk factors and policy implications. Journal of Studies on Alcohol, 63(2), 129-140.

Glynn, R.J., LoCastro, J.S., Hermos, J.A., \& Bosse, R. (1983). Social contexts and motives for drinking in men. Journal of Studies on Alcohol, 44, 1011-1025.

Goldman, M.S. (1994). The alcohol expectancy concept: Applications to assessment, prevention, and treatment of alcohol abuse. Applied and Preventative Psychology, 3(3), 131-144.

Goldstein, H.S., Edelberg, R., Meier, C.F., \& Davis, L. (1988). Relationship of blood pressure and heart rate to experienced anger and expressed anger. Psychosomatic Medicine, 50, 321-329.

Goleman, D. (1995). Emotional intelligence. New York: Bantam Books.

Goleman, D. (1998). Working with emotional intelligence. New York: Bantam Books.

Graczyk, P.A., Weissberg, R.P., Payton, J.W., Elias, M.J., Greenberg, M.T., \& Zins, J.E. (2001). Criteria for evaluating the quality of school-based social 
and emotional learning programs. In R. Bar-On \& J. D. A. Parker (Eds.), The Handbook of Emotional Intelligence (pp. 391-410). San Francisco: Jossey-Bass.

Greeley, J., \& Oei, T. (1999). Alcohol and tension reduction. In Leonard, K.E. \& Blane, H.T. (Eds.), Psychological theories of drinking and alcoholism $\left(2^{\text {nd }}\right.$ ed., pp.14-53). New York: The Guilford Press.

Gross, J., \& Levenson, R. W. (1993). Emotional suppression: Physiology, selfreport, and expressive behavior. Journal of Personality and Social Psychology, 64, 970-986.

Haemmerlie, F.M., Montgomery, R.L., \& Cowell, S. (1999). Alcohol abuse by university students and its relationship to sociomoral reasoning. Journal of Alcohol and Drug Education, 44(2), 29-43.

Haines, M., \& Spear, S.F. (1996). Changing the perception of the norm: A strategy to decrease binge drinking among college students. Journal of American College Health, 45, 134-140.

Han, Y.C., \& Pruett, S.B. (1995). Mechanisms of ethanol-induced suppression of a primary antibody response in a mouse model for binge drinking. Journal of Pharmacology and Experimental Therapeutics, 275, 950-957.

Hanson, D.J., \& Engs, R.C. (1992). College students' drinking problems: A national study, 1982-1991. Psychological Reports, 71, 39-42. 
Hawkins, J.D., \& Catalano, R.F. (1992). Communities that care: Action for drug abuse prevention. San Francisco: Jossey-Bass Social and Behavioral Science Series.

Hays, R.D., Merz, J.F., \& Nicholas, R. (1995). Response burden, reliability, and validity of the CAGE, Short Mast, and AUDIT alcohol screening measures. Behavioral Research Methods, Instruments \& Computers, 27, 277-280.

Hedlund, J., \& Sternberg, R.J. (2001). Too many intelligences? Integrating social, emotional, and practical intelligence. In R. Bar-On \& J. D. A. Parker (Eds.), The Handbook of Emotional Intelligence (pp. 136-167). San Francisco: Jossey-Bass.

Hedlund, J.L., \& Vieweg, B.W. (1984). The Michigan Alcoholism Screening Test (MAST): A comprehensive review. Journal of Operational Psychiatry, $15,55-64$.

Helmers, K.F., \& Mente, A. (1999). Alexithymia and health behaviors in healthy male volunteers. Journal of Psychosomatic Research, 47(6), 635-645.

Hingson, R., Heeren, T., Zakocs, R., Kopstein, A., \& Wechsler, H. (2002). Magnitude of alcohol-related mortality and morbidity among U.S. college students ages 18-24. Journal of Studies on Alcohol, 63, 136-144.

Hilton, M.E. (1987). Drinking patterns and drinking problems in 1984: Results from the general population survey. Alcoholism, 11, 167-175. 
Horn, J.L., Wanberg, K.W., \& Foster, F.M. (1987). Guide to the Alcohol Use Inventory. Minneapolis, MN: National Computer Systems.

Hurlburt, S.C. \& Sher, K.J. (1992). Assessing alcohol problems in college students. Journal of American College Health, 41, 49-58.

Humara, M. J., \& Sherman, M.F. (1999). Situational determinants of alcohol abuse among Caucasian and African-American college students. Addictive Behaviors, 24(1), 135-138.

Hunt, W.A. (1993). Are binge drinkers more at risk of developing brain damage? Alcohol, 10, 559-561.

Hussong, A.M. (2007). Predictors of drinking immediacy following daily sadness: An application of survival analysis to experience sampling data. Addictive Behaviors, 32(5), 1054-1065.

Ichiyama, M.A., \& Kruse, M.I. (1998). The social contexts of binge drinking among private university freshmen. Journal of Alcohol and Drug Education, 44(1), 18-33.

Impara, J.C., \& Plake, B.S. (Eds.). (2001). BarOn Emotional Quotient Inventory (EQ-i). The fourteenth Mental Measurements Yearbook. (pp. 106-109). Lincoln, Nebraska: Buros Institute.

Ingra, A., \& Moos, R.H. (1979). Alcohol use among college students: Some competing hypotheses. Journal of Youth and Adolescence, 8, 393-405. 
Institute of Medicine, National Academy of Sciences. (1990). Broadening the base of treatment for alcohol problems. Washington, DC: National Academy Press.

Isenhart, C.E. (1990). Further support for the criterion validity of the alcohol use inventory. Psychology of Addictive Behavior, 4(2), 77-81.

Ivis, F.J., Adlaf, E.M., \& Rehm, J. (2000). Incorporating the AUDIT into a general population telephone survey: A methodological experiment. Drug \& Alcohol Dependence, 60, 97-104.

Jones-Webb, R.J., Hsiao, C.Y., \& Hannan, P. (1995). Relationships between socioeconomic status and drinking problems among Black and White men. Alcoholism: Clinical and Experimental Research, 19(3), 623-627.

Kauhanen, J., Julkunen, J., Salonen, J.T. (1992). Coping with inner feelings and stress: Heavy alcohol use in the context of alexithymia. Behavioral Medicine, 18(3), 121-126.

Kessler, R.C., Crum, R.M, Warner, L.A., Nelson, C.B., Schulenberg, J., \& Anthony, J.C. (1997). Lifetime co-occurrence of DSM-III-R alcohol abuse and dependence with other psychiatric disorders in the National Comorbidity Survey. Archives of General Psychiatry, 54(4), 313-321.

Krause, N. (1995). Stress, alcohol use, and depressive symptoms in later life. Gerontologist, 35, 296-307. 
Kim, E.L., Larimer, M.E., Walker, D.D., \& Marlatt, G.A. (1997). Relationship of alcohol use to other health behaviors among college students. Psychology of Addictive Behaviors, 11, 166-173.

Knight, J.R., Wechsler, H., Kou, M., Seibering, M., Weitzman, E.R., \& Schuckit, M.A. (2002). Alcohol abuse and dependence among U.S. college students. Journal of Studies on Alcohol, 63(3), 263-271.

Koopmans, J.R., Slutske, W.S., van Baal, G.C.M, \& Boomsma, D.I. (1999). The influence of religion on alcohol use initiation: Evidence for genotype $\mathrm{X}$ environment interaction. Behavior Genetics, 29(6), 445-453.

Krugman, S. (1995). Male development and the transformation of shame. In Levant, R.F., \& Pollack, W.S. (Eds.), A new psychology of men (pp. 91126). New York: Basic Books.

Labouvie, E.W. (1990). Personality and alcohol and marijuana use: Patterns of convergence in young adulthood. International Journal of Addictions, 25, 237-252.

LaBrie, J.W., Pedersen, E.R, \& Tawalbeh, S. (2007). Classifying risky-drinking college students: another look at the two-week drinker-type categorization. Journal of Studies on Alcohol, 68(1), 86-90.

Lang, A.R., Patrick, C.J., \& Stritzke, W.G. (1999). Alcohol and emotional response: A multidimensional-multilevel analysis. In Leonard, K.E. \& 
Blane, H.T. (Eds.), Psychological theories of drinking and alcoholism $\left(2^{\text {nd }}\right.$ ed., pp. 328-371). New York: The Guilford Press.

Lau, M.A., Pihl, R.O., \& Peteron, J.B. (1995). Provocation, acute alcohol intoxication, cognitive performance, and aggression. Journal of Abnormal Psychology, 104, 150-155.

Lazarus, R.S. (1991). Emotion and adaptation. New York: Oxford University Press.

Leichliter, J.S., Meilman, P.W., Presley, C.A., \& Cashin, J.R. (1998). Alcohol use and related consequences among students with varying levels of involvement in college athletics. Journal of American College Health, 46(6), 257-262.

Lemmens, P., Tan, E.S., \& Knibbe, R.A. (1992). Measuring quantity and frequency of drinking in a general population survey: A comparison of five indices. Journal of Studies on Alcohol, 53, 476-486.

Leonard, K.E., \& Blane, H.T. (1999a). Introduction. In Leonard, K.E. \& Blane, H.T. (Eds.), Psychological theories of drinking and alcoholism $\left(2^{\text {nd }}\right.$ ed., pp.1-13). New York: The Guilford Press.

Leonard, K.E., \& Blane, H.T. (1999b). Conclusion. In Leonard, K.E. \& Blane, H.T. (Eds.), Psychological theories of drinking and alcoholism $\left(2^{\text {nd }}\right.$ ed., pp.456-460). New York: The Guilford Press.

Lesser, I. M. (1985). Current concepts in psychiatry: alexithymia. New England Journal of Medicine, 312(11), 690-692. 
Liu, X., \& Kaplan, H. B. (1996). Gender-related differences in circumstances surrounding initiation escalation of alcohol and other substance use/abuse. Deviant behavior: an interdisciplinary journal, 17, 71-106.

Loas, G., Fremaux, D., Otmani, O., Lecerle, C., \& Delahousse, J. (1997). Is alexithymia a negative factor for maintaining abstinence? A follow-up study. Comprehensive Psychiatry, 38(5), 296-299.

Luczak, S.E., Wall, T.L., Shea, S.H., Byun, S.M., \& Carr, L.G. (2001). Binge drinking in Chinese, Korean and White college students: Genetic and ethnic group differences. Psychology of Addictive Behaviors, 15(4), 306309.

Lumley, M.A., Tomakowsky, J., \& Torosian, T. (1997). The relationship of alexithymia to subjective and biomedical measures of disease. Psychosomatics, 38, 497-502.

Lynch, J.J., Lynch, K.E., \& Friedman, E. (1992). A cry unheard: sudden reductions in blood pressure while talking about feelings of hopelessness and helplessness. Integrative Physiological and Behavioral Science, 27, 151-169.

MADD: DUI deaths increase in 2001. (2002, November 21). USA Today.com. Retrieved November 21, 2002, from http://www.usatoday.com Maggs, J.L.(1997). Alcohol use and binge drinking as goal-directed action during the transition to postsecondary education. In J. Schulenberg and J. L. 
Maggs (Eds.), Health risks and developmental transitions during adolescence (pp. 345-371). New York: Cambridge University Press.

Marklein, M.B. (2002, February 27). Binge drinking's campus toll. USA Today. Retrieved July 19, 2002, from http//:www.usatoday.com

Martinez-Pons, M. (1997). The relation of emotional intelligence with selected areas of personal functioning. Imagination, Cognition and Personality, 17(1), 3-13.

Matthews, G. \& Zeidner, M. (2001). Emotional intelligence, adaptation to stressful encounters, and health outcomes. In J. Ciarrochi, J. P. Forgas, \& J. D. Mayer (Eds.), Emotional intelligence in everyday life: A scientific inquiry (pp. 459-489). Philadelphia: Psychology Press.

Mayer, J.D. (1999, September). Emotional intelligence: popular or scientific psychology [Electronic version]? APA Monitor Online, 30(8). Retrieved August 28, 2001, from http://www.apa.org/monitor/sep99/sp.html Mayer, J.D. (2001). A field guide to emotional intelligence. In J. Ciarrochi, J. P. Forgas, \& J. D. Mayer (Eds.), Emotional intelligence in everyday life: A scientific inquiry (pp. 3-24). Philadelphia: Psychology Press.

Mayer, J.D., Caruso, D.R., \& Salovey, P. (2000). Selecting a measure of emotional intelligence: The case for ability scales. In J. Ciarrochi, J. P. Forgas, \& J. D. Mayer (Eds.), Emotional intelligence in everyday life: A scientific inquiry (pp. 320-342). Philadelphia: Psychology Press. 
Mayer, J.D., Salovey, P., \& Caruso, D.R. (1999). MSCEIT item booklet (research version 1.1). Toronto, Canada: Multi-Health Systems.

Mayfield, D., McLeod, G., \& Hall, P. (1974). The CAGE questionnaire: Validation of a new alcoholism instrument. American Journal of Psychiatry, 131, 1121-1123.

McCabe, S.E. (2002). Gender differences in collegiate risk factors for heavy episodic drinking. Journal of Studies on Alcohol, 63, 49-56.

McCabe, S.E., Boyd, C., Hughes, T.L., \& dArcy, H. (2003). Sexual identity and substance use among undergraduate students. Substance Abuse, 24(2), 7791.

Meilman, P.W. (1993). Alcohol induced sexual behavior on campus. Journal of American College Health, 42(1), 27-31.

Meilman, P.W., Leichliter, J.S., \& Presley, C.A. (1999). Greeks and athletes: Who drinks more? Journal of American College Health, 47(4), 187-190.

Melia, K., Corodimas, K., Ryabinin, A., Wilson, M., \& LeDoux, J. (1994). Ethanol pre-SA treatment selectively impairs classical conditioning of contextual cues: Possible involvement of the hippocampus. Society for Neuroscience Abstracts, 24, 1007.

Mercer, P.W., \& Khavari, K.A. (1990). Are women drinking more like men? An empirical examination of the convergence hypothesis. Alcoholism: Clinical and Experimental Research, 14(4), 461-466. 
Midanik, L.T. Tam, T.W., Greenfield, T.K. \& Caetano, R. (1996). Risk functions for alcohol-related problems in a 1988 U.S. national sample. Addiction, $91,1427-1437$.

Miller, W.R., Tonigan, J.S., \& Longabaugh, R. (1995). The Drinker Inventory of Consequences (DrInC): An instrument for assessing adverse consequences of alcohol abuse (Project MATCH Monograph Series Vol. 4; NIH Publication No. 95-3911). Rockville, MD: National Institute on Alcohol Abuse and Alcoholism.

Mooney, D.K., Fromme, K., Kivlahan, D.R., \& Marlatt, G.A. (1987). Correlates of alcohol consumption: sex, age, and expectancies relate differentially to quantity and frequency. Addictive behaviors, 12, 235-240.

Moos, R.H., Moos, B.S., \& Kulik, J.A. (1977). Behavioral and self-concept antecedents and correlates of college student drinking patterns. International Journal of Addictions, 12, 603-615.

Morand, D.A. (1999). Family size and intelligence revisited: The role of emotional intelligence. Psychological Reports, 84(2), 643-649.

Murgraff, V., White, D., \& Phillips, K. (1996). Moderating binge drinking: It is possible to change behaviour if you plan it in advance. Alcohol and Alcoholism, 31(6), 577-582. 
Murphy, J.G., Hoyme, C.K., Colby, S.M., \& Borsari, B. (2006). Alcohol consumption, alcohol-related problems, and quality of life among college students. Journal of College Student Development, 47(1), 110-121.

Naglieri, J.A., \& Bardos, A.N. (1997). General Ability Measure for Adults (GAMA). Tucson, AZ: National Computer Systems.

Nagoshi, C.T. (1999). Perceived control of drinking and other predictors of alcohol use and problems in a college student sample. Addiction Research, 7(4), 291-306.

National Highway Traffic Safety Administration. (1994). Traffic safety facts 1993: A compilation of motor vehicle crash data from the Fatal Accident Reporting System and the General Estimates System. Washington, DC: Author.

National Institute on Alcohol Abuse and Alcoholism. (1997). Ninth special report to the U.S. Congress on alcohol and health. Washington, D.C.: U.S. Government Printing Office.

Neighbors, C., Fossos, N., Woods, B.A., Fabiano, P., Sledge, M., \& Frost, D. (2007). Social anxiety as a moderator of the relationship between perceived norms and drinking. Journal of Studies on Alcohol and Drugs, 68(1), 91-96.

Neighbors, C., Lee, C.M., Lewis, M.A., Fossos, N., \& Larimer, M.E. (2007). Are social norms the best predictor of outcomes among heavy-drinking college students? Journal of Studies on Alcohol and Drugs, 68(4), 556-565. 
Nezlek, J.B., Pilkington, C.J., \& Bilbro, K.G. (1994). Moderation in excess: Binge drinking and social interaction among college students. Journal of Studies on Alcohol, 55, 342-351.

O’Hare, T., \& Sherrer, M.V. (2006). Stress, recent changes in alcohol consumption level and problem drinking in freshman first offenders. Journal of Human Behavior in the Social Environment, 13(3), 33-50.

O’Neill, S.E., Gilbert, R.P., \& Sher, K.J. (2001). Clinical relevance of heavy drinking during college years: cross-sectional and prospective perspectives. Psychology of Addictive Behaviors, 15(4), 350-359.

Pallazza, R., \& Bar-On, R. (1995). A study of the emotional intelligence of convicted criminals. Unpublished manuscript.

Parker, J.D.A. (2001). Emotional intelligence: Clinical and therapeutic implications. In J. Ciarrochi, J. P. Forgas, \& J. D. Mayer (Eds.), Emotional intelligence in everyday life: A scientific inquiry (pp. 490-504). Philadelphia: Psychology Press.

Parker, J.D.A., \& Taylor, G.J. (1997). Relations between alexithymia, personality and affects. In G.J. Taylor, R.M. Bagby, \& J.D.A. Parker (Eds.), Disorders of affect regulation: Alexithymia in medical and psychiatric illness (pp. 26-45). New York: Cambridge University Press. 
Parker, J.D.A., Taylor, G.J., \& Bagby, R.M. (2001). The relationship between emotional intelligence and alexithymia. Personality and Individual Differences, 30(1), 107-115.

Paul, J. P., Stall, R., \& Bloomfield, K. A. (1991). Gay and alcoholic: Epidemiologic and clinical issues. Alcohol Health \& Research World, 15(2), 151-159.

Perez, S., Eliseo, J., Marin, G., \& Marin, B.V. (1994). Behavioral risk factors: A comparison of Latinos and non-Latino Whites in San Francisco. American Journal of Public Health, 84(6), 971-976.

Plant, M.P., Miller, P., Thornton, C., Plant, M., \& Bloomfield, K. (2000). Life stage, alcohol consumption patterns, alcohol-related consequences, and gender. Substance Abuse, 21(4), 265-281.

Poikolainen, K., Tuulio-Henriksson, A., Aalto-Setala, T., Marttunen, M., \& Lonnqvist, J. (2001). Predictors of alcohol intake and heavy drinking in early adulthood: A 5-year follow-up of 15-19-year-old Finnish adolescents. Alcohol \& Alcoholism, 36(1), 85-88.

Presley, C.A., Leichliter, J.S., \& Meilman, P.W. (1998). Alcohol and drugs on American campuses: A report to college presidents. Third in a series: 1995, 1996, and 1997. Carbondale, IL: The CORE Institute.

Presley, C.A., Meilman, P.W., \& Lyerla, R. (1995). Alcohol and drugs on American college campuses: Use, consequences, and perceptions of the 
campus environment (Vol. 2: 1990-1992). Carbondale, IL: Southern Illinois University at Carbondale.

Quigley, L.A., \& Marlatt, G.A. (1996). Drinking among young adults. Alcohol Health \& Research World, 20(3), 185-191.

Rabow, J., \& Neuman, C.A. (1984). Saturday night live: Chronicity of alcohol consumption among college students. Substance and AlcoholActions/Misuse, 5(1), 1-7.

Riley, H., \& Schutte, N.S. (2003). Low emotional intelligence as a predictor of substance-use problems. Journal of Drug Education, 33(4), 391-396.

Rybakowski, J., Ziolkowski, M., Zasdzka, T., \& Brezinski, R. (1988). High prevalence of alexithymia in male patients with alcohol dependence. Drug and Alcohol Dependence, 21, 133-136.

Sala, F. (2002) Emotional competence inventory: technical manual. Boston: HayGroup.

Salovey, P., Bedell, B.T., Detweiler, J.B., \& Mayer, J.D. (1999). Coping intelligently: Emotional intelligence and the coping process. In C. R. Snyder (Ed.), Coping: The psychology of what works (pp. 141-164). New York: Oxford University Press.

Sax, L.J., Gilmartin, S.K., Keup, J.R., Bryant, A.N., \& Plecha, M. (2002). Findings from the 2001 pilot administration of Your First College Year 
(YFCY): National Norms. Los Angeles: Higher Education Research Institute of UCLA.

Schmitz, J.M., Oswald, L.M., Damin, P., \& Mattis, P. (1995). Situational analysis of coping in substance abusing patients. Journal of Substance Abuse, 7, 189-204.

Schulenberg, J.E., Maggs, J.L., Long, S.W., Sher, K.J., Gotham, H.J., Baer, J.S., et al. (2001). The problem of college drinking: insights from a developmental perspective. Alcoholism: Clinical and Experimental Research, 25(3), 473-477.

Schulenberg, J.E., \& Maggs, J.L. (2002). A developmental perspective on alcohol use and heavy drinking during adolescence and the transition to young adulthood. Journal of Studies on Alcohol, 63(2), 54-71.

Schulenberg, J.E., O’Malley, P.M., Bachman, J. G., Wadsworth, K. N., \& Johnston, L. D. (1996). Getting drunk and growing up: Trajectories of frequent binge drinking during the transition to young adulthood. Journal of Studies on Alcohol, 57, 289-304.

Schulenberg, J., Wadsworth, K.N., O’Malley, P.M., Bachman, J.G., \& Johnston, L.D. (1996). Adolescent risk factors for binge drinking during the transition to young adulthood: Variable- and pattern-centered approaches to change. Developmental Psychology, 32(4), 659-674. 
Selzer, M.L. (1971). The Michigan Alcoholism Screening Test: The quest for a new diagnostic instrument. American Journal of Psychiatry, 127, 16531658.

Shakeshaft, A.P., Bowman, J.A., \& Sanson-Fisher, R.W. (1998). Comparison of three methods to assess binge consumption: One-week retrospective diary, AUDIT, and quantity/frequency. Substance Abuse, 19(4), 191-203.

Shakeshaft, A.P., Bowman, J.A., \& Sanson-Fisher, R.W. (1999). A comparison of two retrospective measures of weekly alcohol consumption: Diary and quantity/frequency index. Alcohol and Alcoholism, 34(4), 636-645.

Shepard, J., \& Brickley, M. (1996). The relationship between alcohol intoxication, stressors and injury in urban violence. British Journal of Criminology, 36, 546-566.

Sifneos, P. E. (1973). The prevalence of alexithymic characteristics in psychosomatic patients. Psychotherapy and Psychosomatics, 22, 255-262.

Silbereisen, R.K., \& Noack, P. (1988). On the constructive role of problem behavior in adolescence. In N. Bulger, A. Caspi, G. Downey, \& M. Moorehouse (Eds.), Person in context: Developmental processes (pp. 152180). Cambridge: Cambridge University Press.

Simpson, T. L., \& Arroyo, J. A. (1998). Coping patterns associated with alcoholrelated negative consequences among college women. Journal of Social and Clinical Psychology, 17(2), 150-166. 
Sinclair, M., McRee, B., \& Babor, T.F. (1992). Evaluation of the reliability of AUDIT. Farmington: University of Connecticut School of Medicine, Alcohol Research Center. Unpublished report.

Sitarenios, G. (1998). EQ-i Item-Total Correlations. Toronto, Canada: MultiHealth Systems.

Sitarenios, G., \& Handley, R. (1998) Analysis of EQ-i and substance abuse data from the U.S. Air Force. Toronto, Canada: Multi-Health Systems.

Skinner, H.A., \& Sheu, W.J. (1982). Reliability of alcohol use indices: The Lifetime Drinking History and the MAST. Journal of Studies on Alcohol, 43, 1157-1170.

Smeaton, G.L., Josiam, B.M, \& Dietrich, U.C. (1998). College students' binge drinking at a beach-front destination during spring break. Journal of American College Health, 46(6), 247-254.

Smith, D. (2002, May). Researchers examine success of youth substance abuse prevention programs. Monitor on Psychology, 33(5), 12.

Smith, M.J., Abbey, A., \& Scott, R.O. (1993). Reasons for drinking alcohol: Their relationship to psychosocial variables and alcohol consumption. International Journal of the Addictions, 28, 881-908.

Sobell, L.C., \& Sobell, M.B. (1995). Alcohol consumption measures. In J.P. Allen \& M. Columbus (Eds.), Assessing alcohol problems: A guide for clinicians and researchers. National Institute on Alcohol Abuse and 
Alcoholism Treatment Handbook Series 4 (pp. 55-73). Bethesda, MD:

U.S. Department of Health and Human Services.

Stacy, A.W., Wildaman, K.F., \& Marlatt, G.A. (1990). Expectancy models of alcohol use. Journal of Personality and Social Psychology, 58, 918-928.

Study: College drinking in 1,400 deaths. (2002, April 9). CNN.com. Retrieved April 9, 2002, from http//:www.cnn.com

Taylor, G. T., \& Bagby, R.M. (2000). An overview of the alexithymia construct. In R. Bar-On \& J. D. A. Parker (Eds.), The Handbook of Emotional Intelligence (pp. 40-67). San Francisco: Jossey-Bass.

Taylor, G. T., Parker, J.D.A., \& Bagby, R.M. (1990). A preliminary investigation of alexithymia in men with psychoactive substance dependence. American Journal of Psychiatry, 147, 1228-1230.

Taylor, G.J. (1997). Substance use disorders. In G. J. Taylor, R. M. Bagby, \& J. D. A. Parker, (Eds.), Disorders of affect regulation: Alexithymia in medical and psychiatric illness (pp. 166-189). New York: Cambridge University Press.

Taylor, S.P., \& Sears, J.D. (1988). The effects of alcohol and persuasive social pressure on human physical aggression. Aggressive Behavior, 14, 237 243. 
The Henry J. Kaiser Family Foundation. (2002, February). Substance use and risky sexual behavior: Attitudes and practices among adolescents and young adults. Survey Snapshot. Menlo Park, CA: Author.

The National Center of Addiction and Substance Abuse (CASA) at Columbia University. (1999). Dangerous liaisons: Substance abuse and sex. New York: Author.

Thom, B. (1986). Sex differences in help seeking for alcohol problems. 1. The barriers to help seeking. Addiction, 81, 777-788.

Thorndike, E.L. (1920). Intelligence and its uses. Harper's Magazine, 140, 227 235.

Tonigan, J.S., \& Miller, W.R. (1993). Assessment and validation of the Drinker Inventory of Consequences (DrInC): A multi-site outpatient and aftercare clinical sample of problem drinkers [Abstract]. Alcoholism: Clinical and Experimental Research, 17, 513.

Trinidad, D.R., \& Johnson, C.A. (2002). The association between emotional intelligence and early adolescent tobacco and alcohol use. Personality and Individual Differences, 32, 95-105.

Tschann, J. M. Adler, N. E., Irwin, C. E., Jr., Millstein, S. G., Turner, R. A., \& Kegeles, S. M. (1994). Initiation of substance use in early adolescence: The roles of pubertal timing and emotional distress. Health Psychology, 13(4), 326-333. 
Turnbull, J.E., \& Gomberg, E.S. (1988). Impact of depressive symptomatology on alcohol problems in women. Alcoholism: Clinical and Experimental Research, 12(3), 374-381.

Turrisi, R., Wiersma, K. A., \& Hughes, K.K. (2000). Binge-drinking-related consequences in college students: role of drinking beliefs and mother-teen communications. Psychology of Addictive Behaviors, 14(4), 342-355.

Valois, R.F., McKeown, R.E., Garrison, C.Z., \& Vincent, M.L. (1995). Correlates of aggressive and violent behaviors among public high school adolescents. Journal of Adolescent Health, 16, 26-34.

Vik, P., Carrello, P., Tate, S.R., \& Field, C. (2000). Progression of consequences among heavy-drinking college students. Psychology of Addictive Behaviors, 14, 91-101.

Volpicelli, J.R. (1987). Uncontrollable events and alcohol drinking. British Journal of Addiction, 82, 381-392.

Walters, S.T., \& Bennett, M.E. (2000). Addressing drinking among students: A review of the empirical literature. Alcoholism Treatment Quarterly, 18, $61-77$

Wanberg, K.W., Horn, J.L., \& Foster, F.M. (1977). A differential assessment model of alcoholism. Journal of Studies on Alcohol, 38, 512-543.

Wechsler, H., Davenport, A., Dowdall, G.W., Moeykins, B., \& Castillo, S. (1994). Health and behavioral consequences of binge drinking in college: 
A national survey of students at 140 campuses. Journal of the American Medical Association, 272, 1672-1677.

Wechsler, H., Dowdall, G.W., Davenport, A., \& Rimm, E.B. (1994). A genderspecific measure of binge drinking among college students. American Journal of Public Health, 85, 982-985.

Wechsler, H., Dowdall, G.W., Maenner, B.S., Gledhill-Hoyt, J., \& Lee, H. (1998). Changes in binge drinking and related problems among American college students between 1993 and 1997: results of the Harvard School of Public Health College Alcohol Study. Journal of American College Health, 47, 57-68.

Wechsler, H., Isaac, N.E., Grodstein, F., \& Sellers, D.E. (1994). Continuation and initiation of alcohol use from the first to the second year of college. Journal of Studies on Alcohol, 55(1), 41-45.

Wechsler, H., Kelley, K., Weitzman, E., SanGiovanni, J.P., \& Seibring, M. (2000). What colleges are doing about student binge drinking: A survey of college administrators. Journal of American College Health, 48, 219-226.

Wechsler, H., \& Kuo, M. (2000). College students define binge drinking and estimate its prevalence: Results of a national survey. Journal of American College Health, 49, 57-64.

Wechsler, H., Lee, J.E., Kuo, M., Seibring, M., Nelson, T.F., \& Lee, H. (2002). Trends in college binge drinking during a period of increased prevention 
efforts: Findings from 4 Harvard School of Public Health college alcohol surveys: 1993-2001. Journal of American College Health, 50(5), 203-217. Wechsler, H., Lee, J.E., Nelson, T.F., \& Kuo, M. (2002). Underage college students'drinking behavior, access to alcohol, and the influence of deterrence policies: Findings from the Harvard School of Public Health college alcohol study. Journal of American College Health, 50(5), 223-236.

Wechsler, H., Moeykens, B., Davenport, A., Castillo, S., \& Hansen, J. (1995). The adverse impact of heavy episodic drinkers on other college students. Journal of Studies on Alcohol, 56, 628-634.

Weingardt, H.L., Baer, J.S., Kivlahan, D.R., Roberts, L.J., Miller, E.T., \& Marlett, G.A. (1998). Episodic heavy drinking among college students: methodological issues and longitudinal perspectives. Psychology of Addictive Behaviors, 12, 155-167.

Wells, S., Speechley, M., Koval, J.J., \& Graham, K. (2007). Gender differences in the relationship between heavy episodic drinking, social roles, and alcohol-related aggression in a U.S. sample of late adolescent and young adult drinkers. American Journal of Drug and Alcohol Abuse, 33(1), 2129.

White, H.R., \& Labouvie, E.W. (1989). Towards the assessment of adolescent problem drinking. Journal of Studies on Alcohol, 50, 30-37. 
Windle, M. (1988). Are those adolescent to early adulthood drinking patterns so discontinuous? A response to Temple and Fillmore. International Journal of Addictions, 23, 907-912.

Wise, T.N., Jani, N.N., Kass, E., Sonnenschein, K., \& Mann, L.S. (1988). Alexithymia: relationship to severity of medical illness and depression. Psychotherapy and Psychosomatics, 50, 68-71.

Ylvisaker, M., Szekeres, S.F., \& Feeney, T. (1998). Cognitive rehabilitation: Executive functions. In M. Ylvisaker (Ed.), Traumatic brain injury rehabilitation: Children and adolescents (2nd ed., pp. 221-269). Newton, MA: Butterworth-Heinemann.

Yu, J. (2003). The association between parent alcohol-related behaviors and children's drinking. Drug and Alcohol Dependence, 69(3), 253-262.

Ziemelis, A., Bucknam, R.B., \& Abdulaziz, M.E. (2002). Prevention efforts underlying decreases in binge drinking in institutions of higher education. Journal of American College Health, 50(5), 238-252.

Ziolkowski, M., Gruss, T., \& Rybakowski, J. K. (1995). Does alexithymia in male alcoholics constitute a negative factor for maintaining abstinence? Psychotherapy and Psychosomatics, 63(3-4), 169-173.

Zirkel, S. (2000). Social intelligence. In R. Bar-On \& J. D. A. Parker (Eds.), The Handbook of Emotional Intelligence (pp. 3-27). San Francisco: JosseyBass. 
Ziyadeh, N.J., Prokop, L.A., Fisher, L.B., Rosario, M., Field, A.E., Camargo, C.A., \& Austin, S.B. (2007). Drug and Alcohol Dependence, 87(2-3), 119130. 


\section{APPENDICES}

Appendix A: DSM-IV-TR Criteria for Alcohol Abuse

Appendix B: Research Questionnaire

Appendix C: Examples of the Psychometric Assessments

c.1. BarOn Emotional Quotient Inventory (EQ-i)

c.2. Drinker Inventory of Consequences (DrInC)

Appendix D: Tables

Appendix E: Consent and Information Form 


\section{Appendix A}

The following is the complete diagnostic criteria for Alcohol Abuse as defined by the Diagnostic and Statistical Manual of Mental Disorders ( $4^{\text {th }}$ ed., text rev.; DSM-IV-TR; American Psychiatric Association, 2000):

305.00 Alcohol Abuse

A. A maladaptive pattern of alcohol use leading to clinically significant impairment or distress, as manifested by one (or more) of the following, occurring in a 12-month period:

(1) recurrent alcohol use resulting in a failure to fulfill major role obligations at work, school, or home (e.g., repeated absences or poor work performance related to alcohol use; alcohol-related absences, suspensions, or expulsions from school; neglect of children or household)

(2) recurrent alcohol use in situations in which it is physically hazardous (e.g., driving an automobile or operating a machine when impaired by alcohol use)

(3) recurrent alcohol-related legal problems (e.g., arrests for alcohol-related disorderly conduct)

(4) continued alcohol use despite having persistent or recurrent social or interpersonal problems caused or exacerbated by 
the effects of the substance (e.g., arguments with spouse about consequences of intoxication, physical fights)

B. The symptoms have never met the criteria for Substance Dependence for this class of substance (alcohol). When the aforementioned problems are accompanied by evidence of tolerance, withdrawal, or compulsive behavior related to alcohol use, a diagnosis of Alcohol Dependence, rather than Alcohol Abuse, should be considered. 
Appendix B

Research Questionnaire 
CONFIDENTIAL

Research Questionnaire

Subject \#

Date

Gender: $\quad F \quad M$

Age: $\begin{array}{cc}\text { Under } 18 \\ 21-23\end{array}-\begin{aligned} & 18-20 \\ & -\quad 24 \text { and over } \\ & -\end{aligned}$

Race: (Optional)

Annual household income level: (Optional)

African-American

Below 20,000

Asian-American

Caucasian/White

Hispanic-American or Latino/Latina

20,000-40,000

Native American/American Indian

$40,001-60,000$

Biracial

$60,001-80,000$

Other

$80,001-100,000$

— Above 100,000

Class year:

Freshman (First-year)

Sophomore

Junior

Senior

Are you a member of an intercollegiate athletic team?

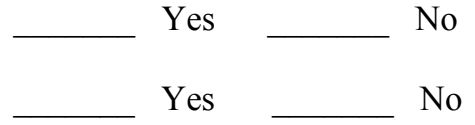

Are you a member of a sorority or fraternity?

No

Please circle the answer that is correct for you:

1. How often do you have a drink containing alcohol?

$\begin{array}{lllll}\text { Never } & \begin{array}{l}\text { Monthly } \\ \text { or less }\end{array} & \begin{array}{l}2 \text { or } 4 \text { times } \\ \text { a month }\end{array} & \begin{array}{l}2 \text { to } 3 \text { times } \\ \text { a week }\end{array} & \begin{array}{l}4 \text { or more times } \\ \text { a week }\end{array}\end{array}$

2. During the past 2 weeks, what was the highest number of drinks containing alcohol that you had on any one occasion?
1 or 2
About 3
About 4
About 5
More than 5

3. FEMALES: How often do you have $\mathbf{4}$ or more drinks of alcohol on one occasion? MALES: How often do you have $\mathbf{5}$ or more drinks of alcohol on one occasion?

$\begin{array}{llll}\text { Never Rarely } \quad \text { Monthly } \quad \text { Every 2 } & \begin{array}{l}\text { Weekly } \\ \text { weeks }\end{array} & \begin{array}{l}\text { Daily or } \\ \text { almost daily }\end{array}\end{array}$


Appendix C

Examples of Psychometric Assessments 


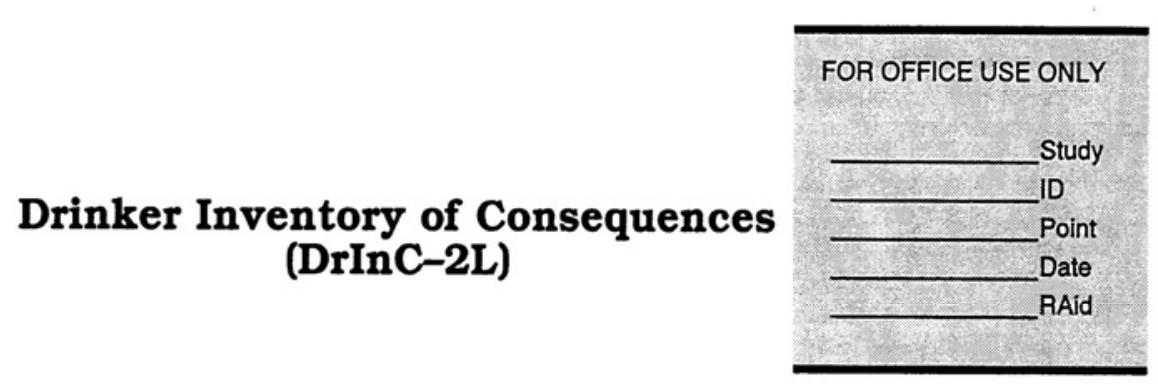

INSTRUCTIONS: Here are a number of events that drinkers sometimes experience. Read each one carefully and circle the number that indicates whether this has EVER happened to you $(0=\mathrm{No}, 1=\mathrm{Yes})$. If an item does not apply to you, circle zero (0).

\begin{tabular}{|c|c|c|}
\hline $\begin{array}{l}\text { Has this EVER happened to you? } \\
\text { Circle one answer for each item. }\end{array}$ & No & Yes \\
\hline 1. I have had a hangover or felt bad after drinking. & 0 & 1 \\
\hline $\begin{array}{l}\text { 2. I have felt bad about myself because of my } \\
\text { drinking. }\end{array}$ & 0 & 1 \\
\hline $\begin{array}{l}\text { 3. I have missed days of work or school because of } \\
\text { my drinking. }\end{array}$ & 0 & 1 \\
\hline $\begin{array}{l}\text { 4. My family or friends have worried or complained } \\
\text { about my drinking. }\end{array}$ & 0 & 1 \\
\hline 5. I have enjoyed the taste of beer, wine, or liquor. & 0 & 1 \\
\hline $\begin{array}{l}\text { 6. The quality of my work has suffered because of } \\
\text { my drinking. }\end{array}$ & 0 & 1 \\
\hline $\begin{array}{l}\text { 7. My ability to be a good parent has been harmed } \\
\text { by my drinking. }\end{array}$ & 0 & 1 \\
\hline $\begin{array}{l}\text { 8. After drinking, I have had trouble with sleeping, } \\
\text { staying asleep, or nightmares. }\end{array}$ & 0 & 1 \\
\hline $\begin{array}{l}\text { 9. I have driven a motor vehicle after having three } \\
\text { or more drinks. }\end{array}$ & 0 & 1 \\
\hline
\end{tabular}


The Drinker Inventory of Consequences (DrInC)

\begin{tabular}{|c|c|c|}
\hline $\begin{array}{l}\text { Has this EVER happened to you? } \\
\text { Circle one answer for each item. }\end{array}$ & No & Yes \\
\hline 10. My drinking has caused me to use other drugs more. & 0 & 1 \\
\hline 11. I have been sick and vomited after drinking. & 0 & 1 \\
\hline 12. I have been unhappy because of my drinking. & 0 & 1 \\
\hline $\begin{array}{l}\text { 13. Because of my drinking, I have not eaten } \\
\text { properly. }\end{array}$ & 0 & 1 \\
\hline $\begin{array}{l}\text { 14. I have failed to do what is expected of me } \\
\text { because of my drinking. }\end{array}$ & 0 & 1 \\
\hline 15. Drinking has helped me to relax. & 0 & 1 \\
\hline $\begin{array}{l}\text { 16. I have felt guilty or ashamed because of my } \\
\text { drinking. }\end{array}$ & 0 & 1 \\
\hline $\begin{array}{l}\text { 17. While drinking, I have said or done embarrassing } \\
\text { things. }\end{array}$ & 0 & 1 \\
\hline $\begin{array}{l}\text { 18. When drinking, my personality has changed for } \\
\text { the worse. }\end{array}$ & 0 & 1 \\
\hline $\begin{array}{l}\text { 19. I have taken foolish risks when I have been } \\
\text { drinking. }\end{array}$ & 0 & 1 \\
\hline 20. I have gotten into trouble because of drinking. & 0 & 1 \\
\hline $\begin{array}{l}\text { 21. While drinking or using drugs, I have said harsh } \\
\text { or cruel things to someone. }\end{array}$ & 0 & 1 \\
\hline $\begin{array}{l}\text { 22. When drinking, I have done impulsive things } \\
\text { that I regretted later. }\end{array}$ & 0 & 1 \\
\hline $\begin{array}{l}\text { 23. I have gotten into a physical fight while } \\
\text { drinking. }\end{array}$ & 0 & 1 \\
\hline $\begin{array}{l}\text { 24. My physical health has been harmed by my } \\
\text { drinking. }\end{array}$ & 0 & 1 \\
\hline $\begin{array}{l}\text { 25. Drinking has helped me to have a more positive } \\
\text { outlook on life. }\end{array}$ & 0 & 1 \\
\hline $\begin{array}{l}\text { 26. I have had money problems because of my } \\
\text { drinking. }\end{array}$ & 0 & 1 \\
\hline & Please & we on the next page. \\
\hline 38 & & \\
\hline
\end{tabular}


The Drinker Inventory of Consequences (DrInC)

\begin{tabular}{|c|c|c|}
\hline $\begin{array}{l}\text { Has this EVER happened to you? } \\
\text { Circle one answer for each item. }\end{array}$ & No & Yes \\
\hline $\begin{array}{l}\text { 27. My marriage or love relationship has been harmed by } \\
\text { my drinking. }\end{array}$ & 0 & 1 \\
\hline $\begin{array}{l}\text { 28. I have smoked tobacco more when I am } \\
\text { drinking. }\end{array}$ & 0 & 1 \\
\hline $\begin{array}{l}\text { 29. My physical appearance has been harmed } \\
\text { by my drinking. }\end{array}$ & 0 & 1 \\
\hline 30. My family has been hurt by my drinking. & 0 & 1 \\
\hline $\begin{array}{l}\text { 31. A friendship or close relationship has } \\
\text { been damaged by my drinking. }\end{array}$ & 0 & 1 \\
\hline $\begin{array}{l}\text { 32. I have been overweight because of my } \\
\text { drinking. }\end{array}$ & 0 & 1 \\
\hline 33. My sex life has suffered because of my drinking. & 0 & 1 \\
\hline $\begin{array}{l}\text { 34. I have lost interest in activities and hobbies } \\
\text { because of my drinking. }\end{array}$ & 0 & 1 \\
\hline $\begin{array}{l}\text { 35. When drinking, my social life has been more } \\
\text { enjoyable. }\end{array}$ & 0 & 1 \\
\hline $\begin{array}{l}\text { 36. My spiritual or moral life has been harmed by } \\
\text { my drinking. }\end{array}$ & 0 & 1 \\
\hline $\begin{array}{l}\text { 37. Because of my drinking, I have not had the kind } \\
\text { of life that I want. }\end{array}$ & 0 & 1 \\
\hline $\begin{array}{l}\text { 38. My drinking has gotten in the way of my growth } \\
\text { as a person. }\end{array}$ & 0 & 1 \\
\hline $\begin{array}{l}\text { 39. My drinking has damaged my social life, } \\
\text { popularity, or reputation. }\end{array}$ & 0 & 1 \\
\hline $\begin{array}{l}\text { 40. I have spent too much or lost a lot of money } \\
\text { because of my drinking. }\end{array}$ & 0 & 1 \\
\hline $\begin{array}{l}\text { 41. I have been arrested for driving under the } \\
\text { influence of alcohol. }\end{array}$ & 0 & 1 \\
\hline
\end{tabular}


The Drinker Inventory of Consequences (DrInC)

\begin{tabular}{|c|c|c|}
\hline $\begin{array}{l}\text { Has this EVER happened to you? } \\
\text { Circle one answer for each item. }\end{array}$ & No & Yes \\
\hline $\begin{array}{l}\text { 42. I have had trouble with the law (other than } \\
\text { driving while intoxicated) because of my drinking. }\end{array}$ & 0 & 1 \\
\hline $\begin{array}{l}\text { 43. I have lost a marriage or a close love } \\
\text { relationship because of my drinking. }\end{array}$ & 0 & 1 \\
\hline $\begin{array}{l}\text { 44. I have been suspended/fired from or left a job or } \\
\text { school because of my drinking. }\end{array}$ & 0 & 1 \\
\hline 45. I drank alcohol normally, without any problems. & 0 & 1 \\
\hline 46. I have lost a friend because of my drinking. & 0 & 1 \\
\hline $\begin{array}{l}\text { 47. I have had an accident while drinking or } \\
\text { intoxicated. }\end{array}$ & 0 & 1 \\
\hline $\begin{array}{l}\text { 48. While drinking or intoxicated, I have been } \\
\text { physically hurt, injured, or burned. }\end{array}$ & 0 & 1 \\
\hline $\begin{array}{l}\text { 49. While drinking or intoxicated, I have injured } \\
\text { someone else. }\end{array}$ & 0 & 1 \\
\hline $\begin{array}{l}\text { 50. I have broken things while drinking or } \\
\text { intoxicated. }\end{array}$ & 0 & 1 \\
\hline
\end{tabular}


Table 3. Subscales of the DrinC and percentage item endorsements for females and males

\begin{tabular}{|c|c|c|c|c|c|c|c|}
\hline [|tomen] & $\begin{array}{l}\mathbf{F} \\
8 \\
\end{array}$ & $\begin{array}{l}M \\
\alpha \\
\end{array}$ & Subscale & (Itten\}) & $\begin{array}{l}\mathbf{F} \\
\%\end{array}$ & $\begin{array}{ll}M \\
r\end{array}$ & Subscale \\
\hline & & & Physlcal consequerrcus & & & & Interpersonal coniequencos [cont.] \\
\hline (1) & 96.29 & 96.0 & 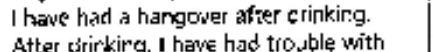 & (17) & 91.5 & 92.6 & $\begin{array}{l}\text { While drinking, I rave said or done } \\
\text { embarrassing things. }\end{array}$ \\
\hline 8 & & & 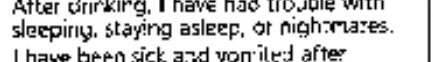 & (21) & 83.98 & 87.7 & $\begin{array}{l}\text { White drinking. I firve wid harsh or cruel } \\
\text { things to someone. }\end{array}$ \\
\hline (11i & git. & 79.8 & $\begin{array}{l}\text { I have been sick. and von iled after } \\
\text { drinking. }\end{array}$ & (27) & $74 \mathrm{c}$ & 89.2 & $\begin{array}{l}\text { My marr age ar lowe relationship has } \\
\text { been härmed ty' my drinki-xy. }\end{array}$ \\
\hline \{13) & 78.1 & 2.2 .2 & $\begin{array}{l}\text { Because of niy drinking. 'have not eaten } \\
\text { property. }\end{array}$ & (30) & 86.3 & $\$ 2.2$ & My fam ty has been hurt hy my drinking. \\
\hline (24) & 75.4 & 79.7 & $\begin{array}{l}\text { My physical health has been harrelec by } \\
\text { my dririking. }\end{array}$ & $\{31\}$ & 68,4 & 76.0 & $\begin{array}{l}\text { A frierdship or close relationship has } \\
\text { been damaged by my drinking. }\end{array}$ \\
\hline (29) & $\pi .27$ & 75.5 & $\begin{array}{l}\text { Ay physical appeatance has been harmed } \\
\text { by miy drinking. }\end{array}$ & $(39)$ & 66.7 & 75.7 & $\begin{array}{l}\text { My drinking has demaged my sacial life, } \\
\text { popularity, or repulatioxi. }\end{array}$ \\
\hline (33) & 51.2 & 67.9 & $\begin{array}{l}\text { Aly sex ife hes sulferec because ot my } \\
\text { driking. }\end{array}$ & $(4 \exists)$ & 34,2 & 51.8 & $\begin{array}{l}\text { I have kist a marriage or a close love } \\
\text { relativorituin because of my drinking. }\end{array}$ \\
\hline (48) & 55.3 & 59.4 & $\begin{array}{l}\text { While drinking or intexicated, I have been } \\
\text { physically inult, injurec, cr butried. }\end{array}$ & (46) & 40.1 & 48.5 & $\begin{array}{l}\text { I have lost a sriend because cf my } \\
\text { diriking. }\end{array}$ \\
\hline Wean & 173.87 & 75.5 & Intrapersonal corrsequences & Mean & 69.7 & 75.2 & impuse control conswquences \\
\hline (2) & 97.7 & 96 म & $\begin{array}{l}\text { i have felt bad abo st mysal' because of } \\
\text { my drinking. }\end{array}$ & (9) & 77.5 & 92.5 & $\begin{array}{l}\text { I hase crives a motor wehicle after } \\
\text { has iny three or mare drioks. }\end{array}$ \\
\hline (12) & 97.1 & 96.1 & $\begin{array}{l}\text { I have been untappy becausc of } \mathrm{my} \\
\text { drinking. }\end{array}$ & (10) & 32.5 & 31.4 & $\begin{array}{l}\text { Vy drinking hass caused me to use cther } \\
\text { drugs more. }\end{array}$ \\
\hline (16) & 96.2 & 94.1 & $\begin{array}{l}\text { I have felt guilty or asinamed because of } \\
\text { my drinkirg. }\end{array}$ & (19) & 77.5 & 88.8 & $\begin{array}{l}\text { I have taken foolish risks when I have } \\
\text { oetn drinking. }\end{array}$ \\
\hline (a) & 25.4 & 83.8 & $\begin{array}{l}\text { 'Nhen dr riking, my pessonality' has } \\
\text { changed fo- the worse. }\end{array}$ & (22) & 82.5 & as.á & $\begin{array}{l}\text { When drinking, I have done impulsive } \\
\text { things that I regretted later. }\end{array}$ \\
\hline (34) & 74.38 & 8C.4 & $\begin{array}{l}\text { nave lost interest In activities and } \\
\text { habbies because of iny drirking. }\end{array}$ & (23) & 42.4 & 50.5 & $\begin{array}{l}\text { I have gotten into a physical figint } \\
\text { while drinungy }\end{array}$ \\
\hline$(36)$ & 69.37 & 75.9 & $\begin{array}{l}\text { My spiritual or molal life hes been } \\
\text { narmed by my drinkirg. }\end{array}$ & (28) & 68.7 & 74.0 & I nave spoked more when I bm drinking. \\
\hline (3T) & 6E. 18 & 89.5 & $\begin{array}{l}\text { Becouse of my drinking, I have not nad } \\
\text { the kirst of life that I want. }\end{array}$ & $(32)$ & 32.6 & 43.7 & 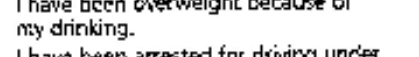 \\
\hline (3B) & 85.78 & 8B.5 & $\begin{array}{l}\text { My deinking as gotten in the syay of my } \\
\text { growth as a person. }\end{array}$ & $\{41\rangle$ & 32.2 & 53.0 & $\begin{array}{l}\text { I have been arrested for drivisy uncer } \\
\text { the influence of alcohtl. }\end{array}$ \\
\hline Mear & 85.4 & 88.1 & Social responsibiltty consequencas & $(42 ;$ & $2 \AA . \bar{c}$ & 40.7 & $\begin{array}{l}\text { I have had trouble with the law (other } \\
\text { than driving winile intoxicatod) because } \\
\text { of my drinking. }\end{array}$ \\
\hline (3) & 60.2 & 68.4 & $\begin{array}{l}\text { I hawe missed days of work or 5chad } \\
\text { berolsse of my drinking. }\end{array}$ & (47) & 36.5 & 52.7 & $\begin{array}{l}\text { I have had ail acoident while drinking } \\
\text { or intoxicated. }\end{array}$ \\
\hline (6) & 67.0 & 72.6 & $\begin{array}{l}\text { The quality of my work has st.fiered } \\
\text { betsuse of my drinking. }\end{array}$ & (49ị & 20.8 & 30.0 & $\begin{array}{l}\text { While drisking or intoxisated, I have } \\
\text { injured somecre else. }\end{array}$ \\
\hline$\{14\}$ & 83.3 & 85.3 & $\begin{array}{l}\text { I have sailed to do what is expected of } \\
\text { me bectuste of my drinkinz. }\end{array}$ & (50) & 57.3 & 56.1 & $\begin{array}{l}\text { I Fave jrekent things or damaged } \\
\text { properly while drinking of ntoxicoted. }\end{array}$ \\
\hline (20) & 50.2 & 5.2 & $\begin{array}{l}\text { Thave gotten into troutle becaust of } \\
\text { drinking. }\end{array}$ & Mean & ก 50.2 & 59.5 & \\
\hline (26) & 56.7 & 79.5 & $\begin{array}{l}\text { I have nat mores' protiems because of } \\
\text { myy drinking. }\end{array}$ & & & 95.9 & $\begin{array}{l}\text { control (raversa-scored validity) itwens } \\
\text { I have enioyed the laste of wine, }\end{array}$ \\
\hline (40) & 71.3 & 87.9 & $\begin{array}{l}\text { Thave spent too much or lost a bot of } \\
\text { money because of my d:inking. }\end{array}$ & (5) & 94.1 & 95.9 & \\
\hline$\{44 j$ & 26.6 & $\begin{array}{l}41.5 \\
73.5\end{array}$ & $\begin{array}{l}\text { I howe been suspended/fired from or left } \\
\text { a job or schod be-ause of my drinking. }\end{array}$ & $\begin{array}{l}(15) \\
(25)\end{array}$ & $\begin{array}{l}95.5 \\
29.8\end{array}$ & 30.8 & $\begin{array}{l}\text { Orinking has helped ree to selax. } \\
\text { Dilnking nas helped the to have a } \\
\text { more vositive outlosk on life. }\end{array}$ \\
\hline & & & $\begin{array}{l}\text { Interpersonal consequencos } \\
\text { My family or friends have worries or }\end{array}$ & (95) & 72.1 & 71.7 & $\begin{array}{l}\text { When drinkmgh, my sacial life has been } \\
\text { more enjoy?tele. }\end{array}$ \\
\hline [4] & & & $\begin{array}{l}\text { complained about my drinking. } \\
\text { Mes ab ity ro be a cood parent has ben }\end{array}$ & (45) & 30.1 & 33.5 & $\begin{array}{l}\text { I drank alcohel tormslly, without any } \\
\text { problems. }\end{array}$ \\
\hline (7) & 54. & & 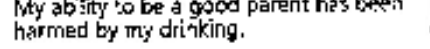 & , Mean & ก 64.4 & 65.4 & \\
\hline
\end{tabular}




\section{Appendix D}

Tables 
Table 1

Reliability and Validity of the Drinker Inventory of Consequences (DrInc)

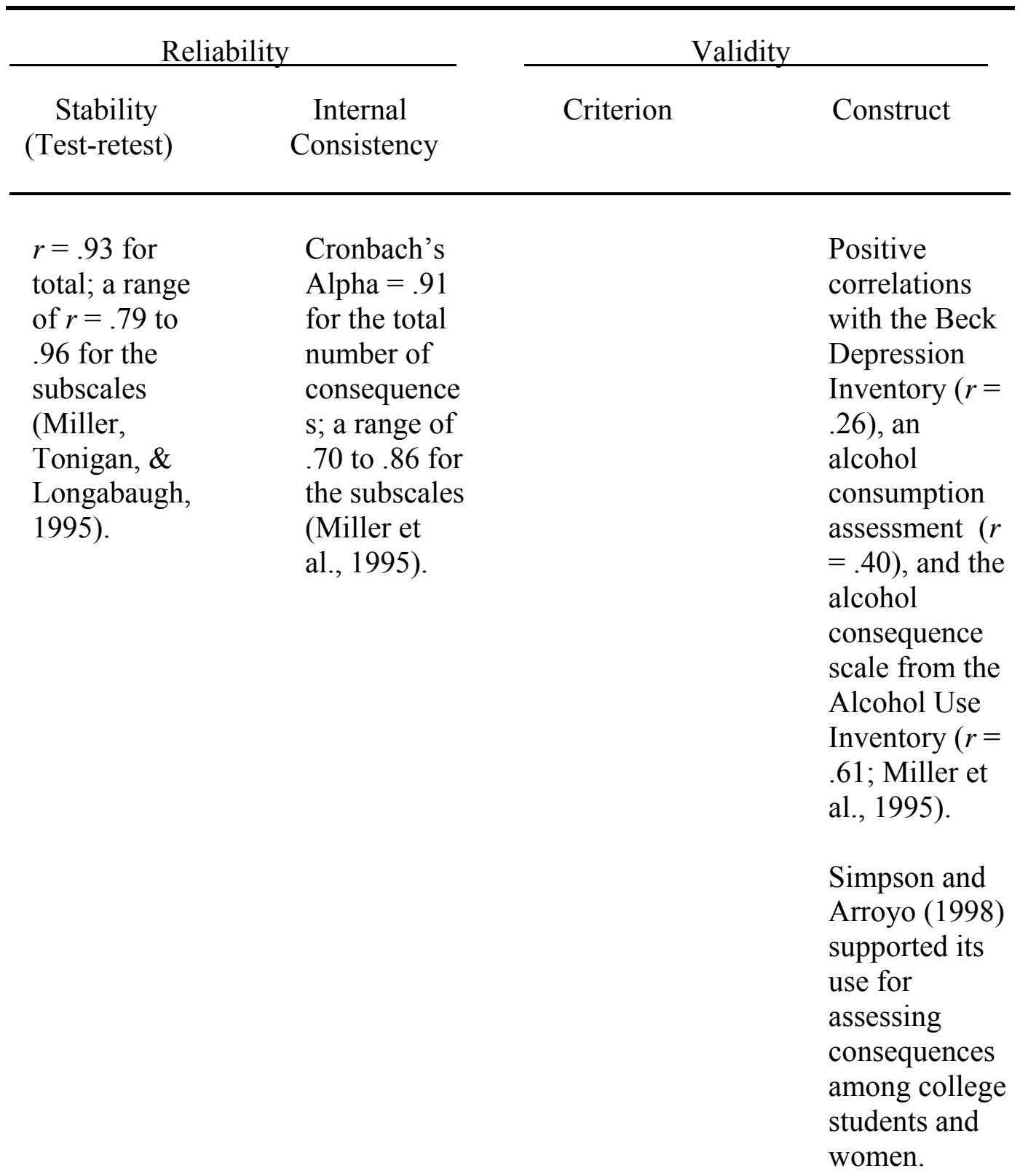


Table 2

Reliability and Validity of the BarOn Emotional Quotient Inventory (EQ-i)

\begin{tabular}{|c|c|c|c|}
\hline \multicolumn{2}{|c|}{ Reliability } & \multicolumn{2}{|c|}{ Validity } \\
\hline $\begin{array}{c}\text { Stability } \\
\text { (Test-retest) }\end{array}$ & $\begin{array}{l}\text { Internal } \\
\text { Consistency }\end{array}$ & Criterion & Construct \\
\hline $\begin{array}{l}r=.85 \text { at one- } \\
\text { month; } r=.75 \text { at } \\
\text { three-months } \\
\text { (Bar-On, 2002). }\end{array}$ & $\begin{array}{l}\text { Nine studies } \\
\text { report a range of } \\
\text { scores from } r= \\
.69 \text { to } .86 \text { (Bar- } \\
\text { On, 2002). } \\
\text { Item-total } \\
\text { correlations were } \\
\text { greater than } r= \\
.30 \text { for each } \\
\text { factor (Sitarenios, } \\
\text { 1998). } \\
\text { Cronbach's } \\
\text { Alpha }=.96 \text { for } \\
\text { total score among } \\
\text { both college men } \\
\text { and women. } \\
\text { Lowest } \\
\text { Cronbach's } \\
\text { Alpha score for } \\
\text { any factor or } \\
\text { subfactor was } \\
.71 . \text { All factors } \\
\text { were correlated } \\
\text { with the total } \\
\text { score within the } \\
\text { range of } r=.67 \\
\text { to .93 (Dawda \& } \\
\text { Hart, 2000). }\end{array}$ & $\begin{array}{l}\text { Criterion validity } \\
\text { established with } \\
\text { samples from } \\
\text { Young President's } \\
\text { Organization, } \\
\text { persons chronically } \\
\text { unemployed, } \\
\text { psychologists, and } \\
\text { financial services } \\
\text { industry workers } \\
\text { (Bar-On, 2002). }\end{array}$ & $\begin{array}{l}\text { Ten assessments } \\
\text { of emotional and } \\
\text { interpersonal } \\
\text { functioning were } \\
\text { significantly } \\
\text { correlated from } \\
r=.30 \text { to } r=.70 \\
\text { (Bar-On, 2002). } \\
\text { A correlation of } r \\
=.08 \text { with the } \\
\text { GAMA } \\
\text { (Derksen, } \\
\text { Kramer, \& } \\
\text { Katsko, 2002). } \\
\text { Negative } \\
\text { correlation with } \\
\text { the Twenty-item } \\
\text { Toronto } \\
\text { Alexithymia } \\
\text { Scale (Parker et } \\
\text { al., 2001). } \\
\text { Significant } \\
\text { correlations for the } \\
\text { EQ-i and measures } \\
\text { of personality, } \\
\text { symptom } \\
\text { presentation, and } \\
\text { affect/mood } \\
\text { (Dawda \& Hart, } \\
\text { 2000). }\end{array}$ \\
\hline
\end{tabular}


Table 3

Demographic Frequencies and Percentages of Participants $(N=309)$

\begin{tabular}{lll}
\hline Variable & Frequency & Percent \\
\hline
\end{tabular}

Age

$\begin{array}{lcc}\text { Under } 18 & 0 & 0 \\ 18-20 & 146 & 47.2 \\ 21-23 & 127 & 41.1 \\ 24 \text { and over } & 36 & 11.7\end{array}$

Athlete Status

Athlete

Non-athlete

59

250

19.1

80.9

Class Year

$\begin{array}{lcc}\text { First-year } & 55 & 17.8 \\ \text { Sophomore } & 73 & 23.6 \\ \text { Junior } & 70 & 22.7 \\ \text { Senior } & 111 & 35.9\end{array}$

Gender

$\begin{array}{lll}\text { Female } & 198 & 64.1 \\ \text { Male } & 111 & 35.9\end{array}$

Greek System

$\begin{array}{lcc}\text { Member } & 52 & 16.8 \\ \text { Nonmember } & 257 & 83.2\end{array}$




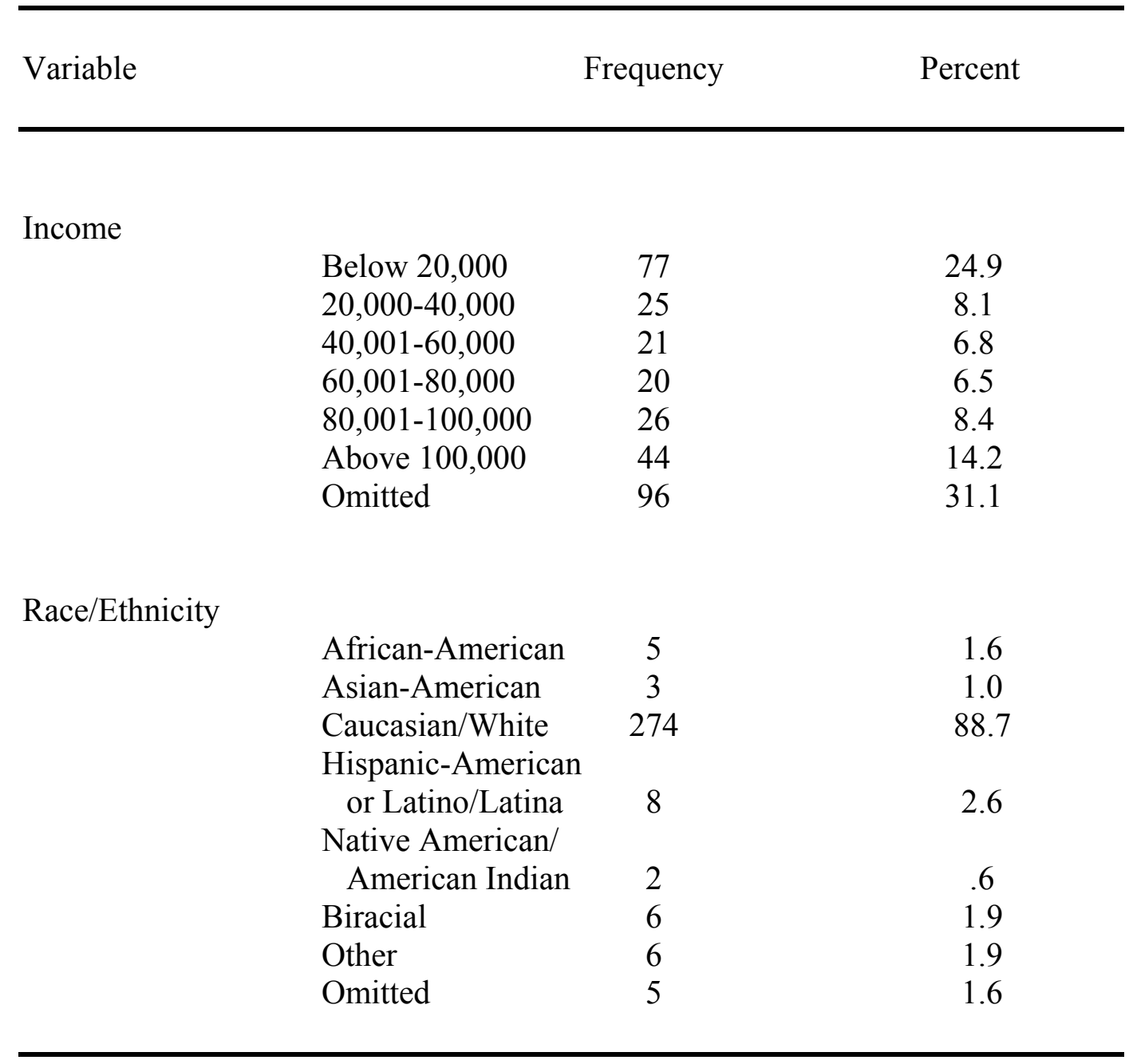


Table 4

EQ-i Means, Standard Deviations, Standard Error Scores, and Ranges for Age

\begin{tabular}{|c|c|c|c|c|}
\hline Subgroup & Mean & SD & $\mathrm{SE}$ & Range \\
\hline $\begin{array}{l}18-20 \\
(\mathrm{~N}=146)\end{array}$ & 96.1 & 12.5 & 1.0 & $65-125$ \\
\hline $\begin{array}{l}21-23 \\
(\mathrm{~N}=127)\end{array}$ & 98.1 & 12.8 & 1.1 & $51-123$ \\
\hline $\begin{array}{l}24 \text { and over } \\
(\mathrm{N}=36)\end{array}$ & 94.7 & 13.5 & 2.3 & $58-119$ \\
\hline
\end{tabular}


Table 5

EQ-i Means, Standard Deviations, Standard Error Scores, and Ranges for Athlete

\begin{tabular}{lcccc}
\hline Subgroup & Mean & SD & SE & Range \\
\hline $\begin{array}{l}\text { Athlete } \\
(\mathrm{N}=59)\end{array}$ & 99.0 & 13.0 & 1.7 & $58-125$ \\
$\begin{array}{l}\text { Non-athlete } \\
(\mathrm{N}=250)\end{array}$ & 96.2 & 12.6 & .8 & $51-123$ \\
\hline $\begin{array}{l}\text { Note. The difference in the mean scores is not significant. } \\
\text { n }\end{array}$
\end{tabular}


Table 6

EQ-i Means, Standard Deviations, Standard Error Scores, and Ranges for Class

\begin{tabular}{|c|c|c|c|c|}
\hline Subgroup & Mean & SD & $\mathrm{SE}$ & Range \\
\hline $\begin{array}{l}\text { First-year } \\
(\mathrm{N}=55)\end{array}$ & 94.7 & 13.3 & 1.8 & $65-120$ \\
\hline $\begin{array}{l}\text { Sophomore } \\
(\mathrm{N}=73)\end{array}$ & 96.6 & 13.3 & 1.6 & $66-125$ \\
\hline $\begin{array}{l}\text { Junior } \\
(\mathrm{N}=70)\end{array}$ & 98.4 & 10.9 & 1.3 & $74-121$ \\
\hline $\begin{array}{l}\text { Senior } \\
(\mathrm{N}=111)\end{array}$ & 96.9 & 13.1 & 1.2 & $51-123$ \\
\hline
\end{tabular}

Note. The difference in the mean scores is not significant. 
Table 7

EQ-i Means, Standard Deviations, Standard Error Scores, and Ranges for Gender

\begin{tabular}{lcccc}
\hline Subgroup & Mean & SD & SE & Range \\
\hline $\begin{array}{l}\text { Females } \\
(\mathrm{N}=121)\end{array}$ & 95.4 & 12.3 & .9 & $51-122$ \\
$\begin{array}{l}\text { Males } \\
(\mathrm{N}=86)\end{array}$ & 99.1 & 13.3 & 1.3 & $58-125$ \\
\hline Note. The difference in the mean scores is significant $(p<.05)$. &
\end{tabular}


Table 8

EQ-i Means, Standard Deviations, Standard Error Scores, and Ranges for Greek System Membership

\begin{tabular}{lcccc}
\hline Subgroup & Mean & SD & SE & Range \\
\hline $\begin{array}{l}\text { Greek Member } \\
(\mathrm{N}=52)\end{array}$ & 99.7 & 13.0 & 1.8 & $58-125$ \\
$\begin{array}{l}\text { Nonmember } \\
(\mathrm{N}=256)\end{array}$ & 96.2 & 12.6 & .8 & $51-125$ \\
\hline $\begin{array}{l}\text { Note. The difference in the mean scores is not significant. } \\
\end{array}$
\end{tabular}


Table 9

Binge Drinker Sample Sizes, Means, Standard Deviations, and Standard Error Scores for Binge Drinking Frequency by Age

\begin{tabular}{lcccc}
\hline Subgroup & $\mathrm{N}$ & Mean & SD & SE \\
\hline $18-20$ & 99 & 4.3 & .9 & $9.3 \mathrm{E}-02$ \\
$21-23$ & 90 & 4.2 & 1.0 & .1 \\
24 and over & 18 & 3.7 & 1.3 & .3 \\
\hline
\end{tabular}

Note. The difference in the mean scores is not significant. 
Table 10

Binge Drinker Sample Sizes, Means, Standard Deviations, and Standard Error Scores for Binge Drinking Frequency by Athlete Status

\begin{tabular}{lcccc}
\hline Subgroup & $\mathrm{N}$ & Mean & SD & SE \\
\hline Athlete & 43 & 4.5 & .9 & .1 \\
Non-athlete & 164 & 4.1 & 1.0 & $7.9 \mathrm{E}-02$ \\
\hline
\end{tabular}

Note. The difference in the mean scores is not significant. 
Table 11

Binge Drinker Sample Sizes, Means, Standard Deviations, and Standard Error Scores for Binge Drinking Frequency by Class

\begin{tabular}{lcccc}
\hline Subgroup & $\mathrm{N}$ & Mean & $\mathrm{SD}$ & $\mathrm{SE}$ \\
\hline First-year & 32 & 4.4 & 1.0 & .2 \\
Sophomore & 51 & 4.3 & 1.0 & .1 \\
Junior & 50 & 4.2 & .8 & .1 \\
Senior & 74 & 4.1 & 1.1 & .1 \\
\hline
\end{tabular}

Note. The difference in the mean scores is not significant. 
Table 12

Binge Drinker Sample Sizes, Means, Standard Deviations, and Standard Error Scores for Binge Drinking Frequency by Gender

\begin{tabular}{lcccc}
\hline Subgroup & $\mathrm{N}$ & Mean & SD & SE \\
\hline Females & 121 & 4.1 & 1.0 & $9.4 \mathrm{E}-02$ \\
Males & 86 & 4.4 & .9 & .1 \\
\hline
\end{tabular}

Note. The difference in the mean scores is not significant. 
Table 13

Binge Drinker Sample Sizes, Means, Standard Deviations, and Standard Error Scores for Binge Drinking Frequency by Greek Membership

\begin{tabular}{lcccc}
\hline Subgroup & $\mathrm{N}$ & Mean & SD & SE \\
\hline Greek Member & 42 & 4.3 & 1.0 & .2 \\
Nonmember & 165 & 4.2 & 1.0 & $7.8 \mathrm{E}-02$ \\
\hline
\end{tabular}

Note. The difference in the mean scores is not significant. 
Table 14

Binge Drinking Consequence (DrInC Total Scores) Means, Standard Deviations, Standard Error Scores, and Ranges for Age

\begin{tabular}{lllll}
\hline Subgroup & Mean & SD & SE & Range \\
\hline $\begin{array}{l}18-20 \\
(\mathrm{~N}=99)\end{array}$ & 11.5 & 6.3 & .6 & $0-29$ \\
$\begin{array}{l}21-23 \\
(\mathrm{~N}=90)\end{array}$ & 13.8 & 8.0 & .8 & $2-36$ \\
$\begin{array}{l}24 \text { and over } \\
(\mathrm{N}=18)\end{array}$ & 17.7 & 8.6 & 2.0 & $7-38$ \\
$\begin{array}{l}\text { Note. The difference among the mean scores is significant }(p<.01) . \text { See Table } 19 \text { for the } \\
\text { statistical analyses. }\end{array}$ &
\end{tabular}


Table 15

Binge Drinking Consequence (DrInC Total Scores) Means, Standard Deviations, Standard Error Scores, and Ranges for Athlete Status

\begin{tabular}{lcccc}
\hline Subgroup & Mean & SD & SE & Range \\
\hline $\begin{array}{l}\text { Athlete } \\
(\mathrm{N}=43)\end{array}$ & 12.9 & 6.5 & 1.0 & $4-34$ \\
$\begin{array}{l}\text { Non-athlete } \\
(\mathrm{N}=164)\end{array}$ & 13.1 & 7.6 & .6 & $0-38$ \\
$\begin{array}{l}\text { Note. The difference in the mean scores is not significant. } \\
\text { Not }\end{array}$
\end{tabular}


Table 16

Binge Drinking Consequence (DrInC Total Scores) Means, Standard Deviations, Standard Error Scores, and Ranges for Class

\begin{tabular}{|c|c|c|c|c|}
\hline Subgroup & Mean & SD & $\mathrm{SE}$ & Range \\
\hline $\begin{array}{l}\text { First-year } \\
(\mathrm{N}=32)\end{array}$ & 9.9 & 6.2 & 1.1 & $0-25$ \\
\hline $\begin{array}{l}\text { Sophomore } \\
(\mathrm{N}=51)\end{array}$ & 11.9 & 7.0 & 1.0 & $2-38$ \\
\hline $\begin{array}{l}\text { Junior } \\
(\mathrm{N}=50)\end{array}$ & 12.9 & 6.6 & .9 & $2-32$ \\
\hline $\begin{array}{l}\text { Senior } \\
(\mathrm{N}=74)\end{array}$ & 15.3 & 8.1 & 1.0 & $2-37$ \\
\hline
\end{tabular}


Table 17

Binge Drinking Consequence (DrInC Total Scores) Means, Standard Deviations, Standard Error Scores, and Ranges for Gender

\begin{tabular}{lcccc}
\hline Subgroup & Mean & SD & SE & Range \\
\hline $\begin{array}{l}\text { Females } \\
(\mathrm{N}=121)\end{array}$ & 12.9 & 6.9 & .6 & $2-38$ \\
$\begin{array}{l}\text { Males } \\
(\mathrm{N}=86)\end{array}$ & 13.2 & 8.1 & .9 & $0-36$ \\
\hline $\begin{array}{l}\text { Note. The difference in the mean scores is not significant. } \\
\text { n }\end{array}$ &
\end{tabular}


Table 18

Binge Drinking Consequence (DrInC Total Scores) Means, Standard Deviations, Standard Error Scores, and Ranges for Greek Membership

\begin{tabular}{lcccc}
\hline Subgroup & Mean & SD & SE & Range \\
\hline $\begin{array}{l}\text { Greek member } \\
(\mathrm{N}=42)\end{array}$ & 12.8 & 8.6 & 1.3 & $2-36$ \\
$\begin{array}{l}\text { Nonmember } \\
(\mathrm{N}=165)\end{array}$ & 13.1 & 7.2 & .6 & $0-38$ \\
$\begin{array}{l}\text { Note. The difference in the mean scores is not significant. } \\
\text { Not }\end{array}$
\end{tabular}


Table 19

Summary Tables of the Statistical Analyses for Differences in Binge Drinking Consequences within Age

\begin{tabular}{lccccc}
\hline Source & SS & df & MS & $F$ & $p$ \\
\hline ANOVA & & & & & \\
Between Groups & 672.07 & 2 & 336.04 & 6.4 & $.002^{*}$ \\
Within Groups & 10802.5 & 204 & $(52.95)$ & & \\
& & & & & \\
\hline
\end{tabular}

Note. Values enclosed in parentheses represent the mean square error.

$* p<.01$.

Tukey HSD Multiple Comparisons for Age

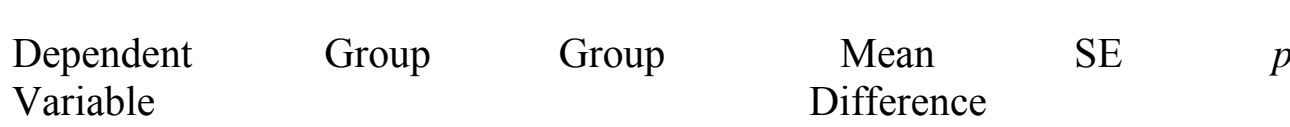

\begin{tabular}{|c|c|c|c|c|c|}
\hline $\begin{array}{l}\text { Binge Drinking } \\
\text { Consequences }\end{array}$ & $18-20$ & $\begin{array}{l}21-23 \\
24 \text { and over }\end{array}$ & $\begin{array}{l}-2.31 \\
-6.16\end{array}$ & $\begin{array}{l}1.06 \\
1.87\end{array}$ & $\begin{array}{l}.075 \\
.003 *\end{array}$ \\
\hline & $21-23$ & $\begin{array}{l}18-20 \\
24 \text { and over }\end{array}$ & $\begin{array}{c}2.31 \\
-3.86\end{array}$ & $\begin{array}{l}1.06 \\
1.88\end{array}$ & $\begin{array}{l}.075 \\
.100\end{array}$ \\
\hline & 24 and over & $\begin{array}{l}18-20 \\
21-23\end{array}$ & $\begin{array}{l}6.16 \\
3.86\end{array}$ & $\begin{array}{l}1.87 \\
1.88\end{array}$ & $\begin{array}{l}.003 * \\
.100\end{array}$ \\
\hline
\end{tabular}


Table 20

Summary Tables of the Statistical Analyses for Differences in Binge Drinking Consequences within Class Year

\begin{tabular}{lccccc}
\hline Source & SS & df & MS & $F$ & $p$ \\
\hline ANOVA & & & & & \\
Between Groups & 765.46 & 3 & 255.16 & 4.8 & $.003^{*}$ \\
Within Groups & 10709.1 & 203 & $(52.75)$ & & \\
\hline
\end{tabular}

Note. Values enclosed in parentheses represent the mean square error.

$* p<.01$.

Tukey HSD Multiple Comparisons for Class Year

\begin{tabular}{llllll}
\hline $\begin{array}{l}\text { Dependent } \\
\text { Variable }\end{array}$ & Group & Group & $\begin{array}{c}\text { Mean } \\
\text { Difference }\end{array}$ & SE & $p$ \\
\hline $\begin{array}{l}\text { Binge Drinking } \\
\text { Consequences }\end{array}$ & First-year & Sophomore & -1.98 & 1.64 & .623 \\
& & Junior & -2.97 & 1.64 & .269 \\
& & Senior & -5.40 & 1.54 & $.002^{* *}$ \\
& \multirow{2}{*}{ Sophomore } & First-year & 1.98 & 1.64 & .623 \\
& & Junior & -1.00 & 1.45 & .901 \\
& & Senior & -3.43 & 1.32 & $.047^{*}$ \\
& \multirow{2}{*}{ Junior } & First-year & 2.97 & 1.64 & .269 \\
& & Sophomore & 1.00 & 1.45 & .901 \\
& & Senior & -2.43 & 1.33 & .260 \\
& \multirow{2}{*}{ Senior } & First-year & 5.40 & 1.54 & $.002^{* *}$ \\
& & Sophomore & 3.43 & 1.32 & $.047^{*}$ \\
& & Junior & 2.43 & 1.33 & .260 \\
\hline
\end{tabular}

${ }^{* p<.05 .}{ }^{* *} p<.01$. 
Table 21

Summary of the Stepwise Regression Analysis for EI Variables Predicting Physical Binge Drinking Consequences $(N=207)$

\begin{tabular}{lcccc}
\hline Variable & $R$ & $R$ Square & $\begin{array}{l}\text { Adjusted } \\
R \text { Square }\end{array}$ & $\begin{array}{l}\text { Standard Error } \\
\text { of the Estimate }\end{array}$ \\
\hline Model 1 & & & & \\
Intrapersonal EI & .31 & .098 & .09 & 1.48 \\
\hline
\end{tabular}


Table 22

ANOVA for the Regression Analysis Model Predicting Physical Consequences

\begin{tabular}{|c|c|c|c|c|c|}
\hline Model & $\mathrm{SS}$ & $\mathrm{df}$ & MS & $F$ & $p$ \\
\hline \multicolumn{6}{|l|}{1} \\
\hline Regression & 48.86 & 1 & 48.86 & 22.25 & $.000 *$ \\
\hline Residual & 450.08 & 205 & 2.20 & & \\
\hline Total & 498.94 & 206 & & & \\
\hline
\end{tabular}


Table 23

Coefficients for the Regression Analysis Model Predicting Physical Consequences $(N=207)$

\begin{tabular}{lccc}
\hline Variable & $B$ & $S E B$ & $\beta$ \\
\hline Model 1 & & & \\
$\quad$ Intrapersonal EI & $-3.4 \mathrm{E}-02$ & .01 & $-.31^{*}$ \\
& & & \\
\hline${ }^{* p<.001 .}$
\end{tabular}


Table 24

Summary of the Stepwise Regression Analysis for EI Variables Predicting

Intrapersonal Binge Drinking Consequences $(N=207)$

\begin{tabular}{lcccc}
\hline Variable & $R$ & $R$ Square & $\begin{array}{c}\text { Adjusted } \\
R \text { Square }\end{array}$ & $\begin{array}{c}\text { Standard Error } \\
\text { of the Estimate }\end{array}$ \\
\hline Model 1 & & & & \\
Stress Management EI & .27 & .07 & .07 & 1.8 \\
\hline
\end{tabular}


Table 25

ANOVA for the Regression Analysis Model Predicting Intrapersonal Consequences

\begin{tabular}{|c|c|c|c|c|c|}
\hline Model & $\mathrm{SS}$ & df & MS & $F$ & $p$ \\
\hline \multicolumn{6}{|l|}{1} \\
\hline Regression & 50.57 & 1 & 50.57 & 15.66 & $.000 *$ \\
\hline Residual & 661.85 & 205 & 3.23 & & \\
\hline Total & 712.42 & 206 & & & \\
\hline
\end{tabular}


Table 26

Coefficients for the Regression Analysis Model Predicting Intrapersonal Consequences $(N=207)$

\begin{tabular}{lccc}
\hline Variable & $B$ & $S E B$ & $\beta$ \\
\hline Model 1 & & & \\
$\quad$ Intrapersonal EI & $-4.0 \mathrm{E}-02$ & .01 & $-.27^{*}$ \\
& & & \\
\hline${ }^{* p<.001 .}$
\end{tabular}


Table 27

Summary of the Stepwise Regression Analysis for EI Variables Predicting Interpersonal Binge Drinking Consequences $(N=207)$

\begin{tabular}{lcccc}
\hline Variable & $R$ & $R$ Square & $\begin{array}{c}\text { Adjusted } \\
R \text { Square }\end{array}$ & $\begin{array}{l}\text { Standard Error } \\
\text { of the Estimate }\end{array}$ \\
\hline Model 1 & & & & \\
$\begin{array}{l}\text { Stress Management EI } \\
\text { Interpersonal EI }\end{array}$ & .37 & .14 & .13 & 1.5 \\
\hline
\end{tabular}


Table 28

ANOVA for the Regression Analysis Model Predicting Interpersonal Consequences

\begin{tabular}{|c|c|c|c|c|c|}
\hline Model & $\mathrm{SS}$ & df & MS & $F$ & $p$ \\
\hline \multicolumn{6}{|l|}{1} \\
\hline Regression & 71.20 & 2 & 35.60 & 16.44 & $.000 *$ \\
\hline Residual & 441.67 & 204 & 2.17 & & \\
\hline Total & 512.87 & 206 & & & \\
\hline
\end{tabular}


Table 29

Coefficients for the Regression Analysis Model Predicting Interpersonal Consequences $(N=207)$

\begin{tabular}{lccc}
\hline Variable & $B$ & $S E B$ & $\beta$ \\
\hline Model 1 & & & \\
& & & \\
$\quad$ Stress Management EI & $-3.8 \mathrm{E}-02$ & .01 & $-.30^{* *}$ \\
$\quad$ Interpersonal EI & $-1.8 \mathrm{E}-02$ & .01 & $-.16^{*}$ \\
& & & \\
\hline${ }^{* p<.05 . * * p<.001 .}$ &
\end{tabular}


Table 30

Summary of the Stepwise Regression Analysis for EI Variables Predicting Impulse Control Binge Drinking Consequences ( $N=207)$

\begin{tabular}{lcccc}
\hline Variable & $R$ & $R$ Square & $\begin{array}{l}\text { Adjusted } \\
R \text { Square }\end{array}$ & $\begin{array}{l}\text { Standard Error } \\
\text { of the Estimate }\end{array}$ \\
\hline Model 1 & & & & \\
$\begin{array}{l}\text { Stress Management EI } \\
\text { Interpersonal EI }\end{array}$ & .31 & .098 & .09 & 2.39 \\
\hline
\end{tabular}


Table 31

ANOVA for the Regression Analysis Model Predicting Impulse Control Consequences

\begin{tabular}{|c|c|c|c|c|c|}
\hline Model & $\mathrm{SS}$ & $\mathrm{df}$ & MS & $F$ & $p$ \\
\hline \multicolumn{6}{|l|}{1} \\
\hline Regression & 126.43 & 2 & 63.22 & 11.11 & $.000 *$ \\
\hline Residual & 1160.82 & 204 & 5.69 & & \\
\hline Total & 1287.26 & 206 & & & \\
\hline
\end{tabular}


Table 32

Coefficients for the Regression Analysis Model Predicting Impulse Control Consequences $(N=207)$

\begin{tabular}{lccc}
\hline Variable & $B$ & $S E B$ & $\beta$ \\
\hline Model 1 & & & \\
& & & \\
$\quad$ Stress Management EI & $-4.7 \mathrm{E}-02$ & .01 & $-.23^{* *}$ \\
$\quad$ Interpersonal EI & $-3.0 \mathrm{E}-02$ & .01 & $-.18^{*}$ \\
& & & \\
\hline${ }^{* p<.05 . * * p<.001 .}$ & &
\end{tabular}


Table 33

Summary of the Stepwise Regression Analysis for EI Variables Predicting Social Responsibility Binge Drinking Consequences ( $N=207)$

\begin{tabular}{lcccc}
\hline Variable & $R$ & $R$ Square & $\begin{array}{l}\text { Adjusted } \\
R \text { Square }\end{array}$ & $\begin{array}{l}\text { Standard Error } \\
\text { of the Estimate }\end{array}$ \\
\hline Model 1 & & & & \\
Interpersonal EI & .85 & .714 & .71 & 8.30 \\
\hline
\end{tabular}


Table 34

ANOVA for the Regression Analysis Model Predicting Social Responsibility Consequences

\begin{tabular}{|c|c|c|c|c|c|}
\hline Model & SS & df & MS & $F$ & $p$ \\
\hline \multicolumn{6}{|l|}{1} \\
\hline Regression & 35300.5 & 1 & 35300.5 & 512.53 & $.000 *$ \\
\hline Residual & 14119.3 & 205 & 68.88 & & \\
\hline Total & 49419.8 & 206 & & & \\
\hline
\end{tabular}


Table 35

Coefficients for the Regression Analysis Model Predicting Social Responsibility Consequences $(N=207)$

\begin{tabular}{lccc}
\hline Variable & $B$ & $S E B$ & $\beta$ \\
\hline Model 1 & & & \\
Intrapersonal EI & .95 & .04 & $.85^{*}$ \\
& & & \\
\hline${ }^{* p<.001 .}$
\end{tabular}


Appendix E

Consent and Information Form 


\section{CONSENT and INFORMATION FORM}

\section{Application of the Emotional Intelligence Construct To College Student Binge Drinking}

\section{Introduction}

I, , am 18 years of age or older and have been invited to participate in this research study which has been explained to me by Jeffrey Dulko. This research is being conducted by Jeffrey Dulko, MA to fulfill the requirements for a doctoral dissertation in the Department of Counseling, Rehabilitation Counseling, and Counseling Psychology at West Virginia University, under supervision of Dr. Margaret Glenn.

\section{Purpose of the Study}

The purpose of this study is to learn more about the psychological factors that may be associated with the negative consequences experienced due to college binge drinking. The results may provide suggestions for improving strategies to reduce and prevent specific types of alcohol-related consequences on college campuses.

\section{Description of Procedures}

I have been instructed by the test administrator, Jeffrey Dulko, to fill out 3 questionnaires about my alcohol drinking habits, consequences from drinking, and emotional functioning which take approximately 45 minutes to complete. I have been told that I may see the questionnaires before signing this consent and that I do not have to answer all the questions if I decide to participate.

Approximately 300 subjects are expected to participate in this study. Upon completion of the questionnaires, I will receive a flier directing me to the available campus resources should I have any further questions regarding alcohol use. I also have the option to submit my name, address, and phone number on a separate sheet for a random drawing of a $\$ 100$ cash prize.

\section{Risks and Discomforts}

There are no known or expected risks from participating in this study, except for the mild frustration which may occur as the result of attempting to answer some of the items on the questionnaires. Answering questions may also result in some discomfort as I reflect on my past behavior.

\section{Alternatives}

I understand that I do not have to participate in this study.

Submission date: 3/20/2006 Page 1 of 2

$\overline{\text { initials }} \overline{\text { date }}$


Application of the Emotional Intelligence Construct To College Student Binge Drinking

\section{Benefits}

I understand that this study is not expected to be of direct benefit to me, but the knowledge gained may be of benefit to others.

\section{Contact Persons}

For more information about this research, I can contact, Jeffrey Dulko, at (585) 322-2731, or his supervisor, Dr. Margaret Glenn at (304) 293-3807. For information regarding my rights as a research subject, I may contact the Office of Research Compliance at (304) 293-7073.

\section{Confidentiality}

I understand that any information about me obtained as a result of my participation in this research will be kept as confidential as legally possible. I understand that my research records and test results, just like hospital records, may be subpoenaed by court order or may be inspected by the study sponsor or federal regulatory authorities (including the FDA if applicable) without my additional consent. In any publications that result from this research, neither my name nor any information from which I might be identified will be published without my consent.

\section{Voluntary Participation}

Participation in this study is voluntary. I understand that I am free to withdraw my consent to participate in this study at any time and that refusal to participate will not affect my class standing or grades. Refusal to participate or withdrawal will involve no penalty to me. I have been given the opportunity to ask questions about the research, and I have received answers concerning areas I did not understand. In the event new information becomes available that may affect my willingness to continue to participate in the study, this information will be given to me so I may make an informed decision about my participation.

Upon signing this form, I will receive a copy.

I willingly consent to participate in this research.

Signature of Subject or
Subject's Legal Representative

Jeffrey Dulko, Investigator

Submission date: 3/20/2006

$\overline{\text { Date }} \overline{\text { Time }}$

$\overline{\text { Date }} \overline{\text { Time }}$

Page 2 of 2 
Curriculum Vitae 


\title{
Jeffrey P. Dulko, MA, CRC, LMHC
}

4 Old Drake Run $\bullet$ Fairport, New York 14450

(585) 322-2731•jdulko@buffalo.edu

\section{Education}

Doctoral Candidate, Counseling Psychology (APA approved) Present WEST VIRGINIA UNIVERSITY • Morgantown, WV

M.A., Rehabilitation Counseling (CORE approved) 1990 NEW YORK UNIVERSITY • New York, NY

B.A., Psychology (Cum Laude), Minor: French 1988

St. BONAVENTURE UNIVERSITY • St. Bonaventure, NY

\section{Certification and License}

\section{Licensed Mental Health Counselor (New York) Certified Rehabilitation Counselor \\ 2006 - Present \\ 1990 - Present}

\section{Rehabilitation and Counseling Experience}

\author{
Associate Director \\ 2007 - Present \\ Senior Trainer \\ $2003-2007$ \\ Region II Rehabilitation Continuing Education Program \\ State UnIVERsity of NEW York • Buffalo, NY \\ Rape Prevention Education Coordinator \\ Acting Clinical Co-coordinator \\ Psychotherapist \\ 1999 - Present \\ $2000-2002$ \\ The Counseling Center \\ $1999-2003$ \\ HobART AND WILLIAM SMITH COLLEGES•Geneva, NY \\ Counseling Psychology Intern (APA approved) 1998 - 1999 \\ Counseling \& Consultation Services \\ UNIVERSITY OF WISCONSIN • Madison, WI \\ Counseling Psychology Doctoral Practicum \\ $1997-1998$ \\ Carruth Center for Counseling and Psychological Services \\ WEST VIRGINIA UNIVERSITY • Morgantown, WV
}


Trauma Therapist Trainee - Doctoral practicum

$1996-1997$

TRAUMA RECOVERY INSTITUTE • Morgantown, WV

Counseling Psychology Trainee - Doctoral practicum 1996

Veterans Administration Medical Center • Clarksburg, WV

Employment Program Supervisor

$1991-1995$

Vocational Counselor/Program Coordinator

Vinland National Center • Loretto, MN

Vocational Evaluator

$1990-1991$

Sister KenNy InStITUTE of ABbott-NORTHWESTERN HOSPITAL

Minneapolis, MN

Rehabilitation Counselor - Internship

$1989-1990$

HiLlside Hospital of LONG ISLAND JeWISH Medical Center

Hillside, NY

Rehabilitation Counselor - Practicum

$1988-1989$

JEWISH GUILD FOR THE BLIND • New York, NY

\section{Undergraduate/Graduate Level Teaching Experience}

Rochester Institute of Technology • Rochester, NY

Course Taught as Adjunct Professor:

Introduction to Psychology

2000

West Virginia University • Morgantown, WV

Courses Taught as Instructor:

Rehabilitation Counseling Practicum

$1997 ; 1998$

Theory and Practice of Human Appraisal

1996; 1997

Case Management with Difficult Clients

1996

Teaching Assistant:

Psychological Aspects of Disability

1996

Medical Aspects of Disability

1995

Counseling Techniques

1995 


\section{Other Related Experience}

Graduate Assistantship

Department of Counseling, Rehabilitation Counseling, and Counseling Psychology

WEST VIRGINIA UNIVERSITY • Morgantown, WV

Counselor/Program Coordinator

NY Lighthouse FOR the Blind VACATION CEnter

Waretown, NJ

Program Assistant (ESSCY Program)

NY LIGHTHOUSE FOR THE BLIND • New York, NY
$1995-1997$

$1988-1990$

$1989-1990$

\section{Publications}

Dulko, J. P. (2006). Maintaining Cohesive Teams in the Midst of Change and Turnover. Technical Assistance Brief (RCEP-II), 2(1).

Dulko, J. P. (2005). Transforming Your Agency Image: Developing Marketing and Public Relation Strategies for New Service Directions. Technical Assistance Brief (RCEP-II), 1(2).

Dulko, J. P. (2005). Situational Assessment: The Art of Designing a New Vocational Service Component. Technical Assistance Brief (RCEP-II), 1(1).

Hird, J. S., Dulko, J. P., Cavalieri, C., Felice, A. A. D., \& Ho, T. A. (2001). Visions and realities: Supervisee perspectives of multicultural supervision. Journal of Multicultural Counseling and Development: Special issue: Multicultural Supervision, 29(2), 114-130.

\section{Literature Review Support}

Marinelli, R. P., \& Dell Orto, A. E. (1999). The Psychological and Social Impact of Disability (4th ed.). New York: Springer Publishing Company.

Cormier, S., \& Cormier, B. (1998). Interviewing Strategies for Helpers: Fundamental Skills and Cognitive Behavioral Interventions (4th ed.). New York: Brooks/Cole Publishing Company. 


\section{Professional Papers, Lectures and Workshops Presented}

Dulko, J. \& Quarles, W. (2006, October). Training Techniques in Employment Settings. Annual Connections and Directions Conference. Atlantic City, NJ.

Dulko, J. (2006, October). Providing Customer Driven Services. Annual Connections and Directions Conference. Atlantic City, NJ.

Dulko, J. \& Duffy, T. (2006, October). Ethical Dilemmas in Supported Employment. Annual Connections and Directions Conference. Atlantic City, NJ.

Dulko, J. \& Quarles, W. (2006, August). Achieving WOW!: The Road from Good to Great. (Invited Training Session). $9^{\text {th }}$ Annual Rehabilitation Services Administration Project Directors' National Conference. Washington, DC.

Dulko, J. (2006, June). Resolving Common Ethical Conflicts in Supported Employment. Association for Persons in Supported Employment National Conference. Boston, MA.

Dulko, J. \& Quarles, W. (2006, May). Providing Customer Driven Services. National Training Conference for VR State Coordinators for the Deaf, Deaf-Blind, Hard of Hearing, and Late-Deafened. Fairport, NY.

Dulko, J. (2006, March). Job Retention for Supported Employees. New Jersey Association for Persons in Supported Employment Annual Conference. Iselin, NJ.

Dulko, J. \& Quarles, W. (2005, September). The Family as a Critical Partner in Achieving Successful Employment Outcomes. Annual Connections and Directions Conference. Atlantic City, NJ.

Dulko, J. (2005, September). Stopping the Slippery Slope: Catching Unethical Behavior Before It Starts. Annual Connections and Directions. Atlantic City, NJ.

Dulko, J. \& Quarles, W. (2005, September). Awareness, beliefs and values. Annual Connections and Directions. Atlantic City, NJ.

Dulko, J. (2005, October). Crisis Management for Direct Service Providers. $4^{\text {th }}$ Annual Allies, Inc. Empowerment, Employment and Choice Conference on Transition. Monroe Township, NJ.

Dulko, J., \& Quarles, W. (2005, August). Customer satisfaction: Strategies for success (Invited Training Session). $8^{\text {th }}$ Annual Rehabilitation Services Administration Project Directors' National Conference. Washington, DC. 
Dulko, J. (2005, July). Stopping the slippery slope: Catching unethical behavior before it starts. Association for Persons in Supported Employment National Conference. Mobile, AL.

Dulko, J., \& Quarles, W. (2005, July). Awareness, beliefs, and values. Association for Persons in Supported Employment National Conference. Mobile, $\mathrm{AL}$.

Dulko, J. (2005, May). Stopping the slippery slope: Catching unethical behavior before it starts. New Jersey Association for Persons in Supported Employment Annual Conference: Facing the Future XIII. Trenton, NJ.

Dulko, J. (2005, April). Crisis management for direct service providers. $A$ State of the Art Forum on Mental Health. Monroe Township, NJ.

Dulko, J. (2005, March). The family as a critical partner in achieving successful employment outcomes (Invited speaker). Nassau Placement Network's $8^{\text {th }}$ Annual Training Day, Westbury, NY.

Dulko, J., \& Quarles, W. (2004, November). Awareness, beliefs, and values. Empire State Association for Persons in Supported Employment Conference. Lake Placid, NY.

Dulko, J. (2004, June). Crisis management. Employment, Empowerment, \& Choice Conference. San Juan, PR.

Dulko, J. (2003, November). Stopping the slippery slope: Catching unethical behavior before it starts. Empire State Association for Persons in Supported Employment Conference. Lake Placid, NY.

Dulko, J. P., Felice, A. A. D., Lund, A. V., \& Slattery, W. S. (1999, February). The Merging of Two Theoretical Cultures in Supervision: A Recipe for Disaster? Big Ten Counseling Centers Conference, Evanston, IL.

Hird, J. S., Dulko, J. P., Cavalieri, C., Felice, A. A. D., \& Ho, T. A. (1999, February). Look Who's Talking Too: Supervisees Discuss Multicultural Supervision. Big Ten Counseling Centers Conference, Evanston, IL.

Dulko, J. P., \& Hinckley, P. (1997, April). Alexithymia and Emotional Numbing. Paper presented at the Treating Traumatic Stress and Dissociative Disorders Conference, West Virginia University School of Medicine, Morgantown, WV.

Tinnin, L., \& Dulko, J. P. (1997, April). Dissociative PTSD. Paper presented at the Treating Traumatic Stress and Dissociative Disorders Conference, West Virginia University School of Medicine, Morgantown, WV. 
Dulko, J. P. (1997, March). Using Myers-Briggs (MBTI) Results in Mediation and Dispute Resolution. Guest lecturer at the West Virginia University College of Law, Morgantown, WV.

Dulko, J. P., \& Kachik, J. R. (1995, October). How to Deal with Difficult People. Workshop presented at the West Virginia University Extension Service, Weston, WV.

Dulko, J. P. (1991, February). The Value and Use of Vocational Evaluation Results in Job Placement. Presentation at the meeting of the Minnesota Job Placement Division, Minneapolis, MN.

Dulko, J. P., McKeon, B., \& Sampels, S. (1990, April). Overcoming Staff Resistance to Implementing Supported Employment Initiatives. Paper formally presented at the American Association for Counseling and Development (AACD) National Conference, Cincinnati, $\mathrm{OH}$.

Dulko, J. P. (1988, April). Improvement of Memory Using Self-Monitoring, Organization, and Operant Conditioning: A Single Subject Design. Paper presented at the Western Pennsylvania Undergraduate Psychology Conference, Meadville, PA.

\section{Awards}

Nominated for the Eisenhart Outstanding Teaching Award (Rochester Institute of Technology; 2000)

Endowed Supplemental Fellowship Award (WVU) in support of doctoral graduate studies. The award recipients are recommended by the department and selected by faculty of the College of Human Resources \& Education. (1995-96, 1996-97, 1997-98)

Vinland Center's Executive Director Award for professional competence in rehabilitation, team leadership, and agency contribution. (1994)

Adrian Levy Scholarship Award (New York University) in recognition of academic achievement and professional potential in the field of rehabilitation counseling. (1990)

\section{Memberships}

American Counseling Association

American Rehabilitation Counselors Association

Kappa Delta Pi (International Honor Society in Education; expired)

Psi Chi (Psychology Honor Society) 